\title{
Application of Adjusted Data in Calculating Fission-Product Decay Energies and Spectra
}

D. C. George

R. J. '.aBauve

T. R. England
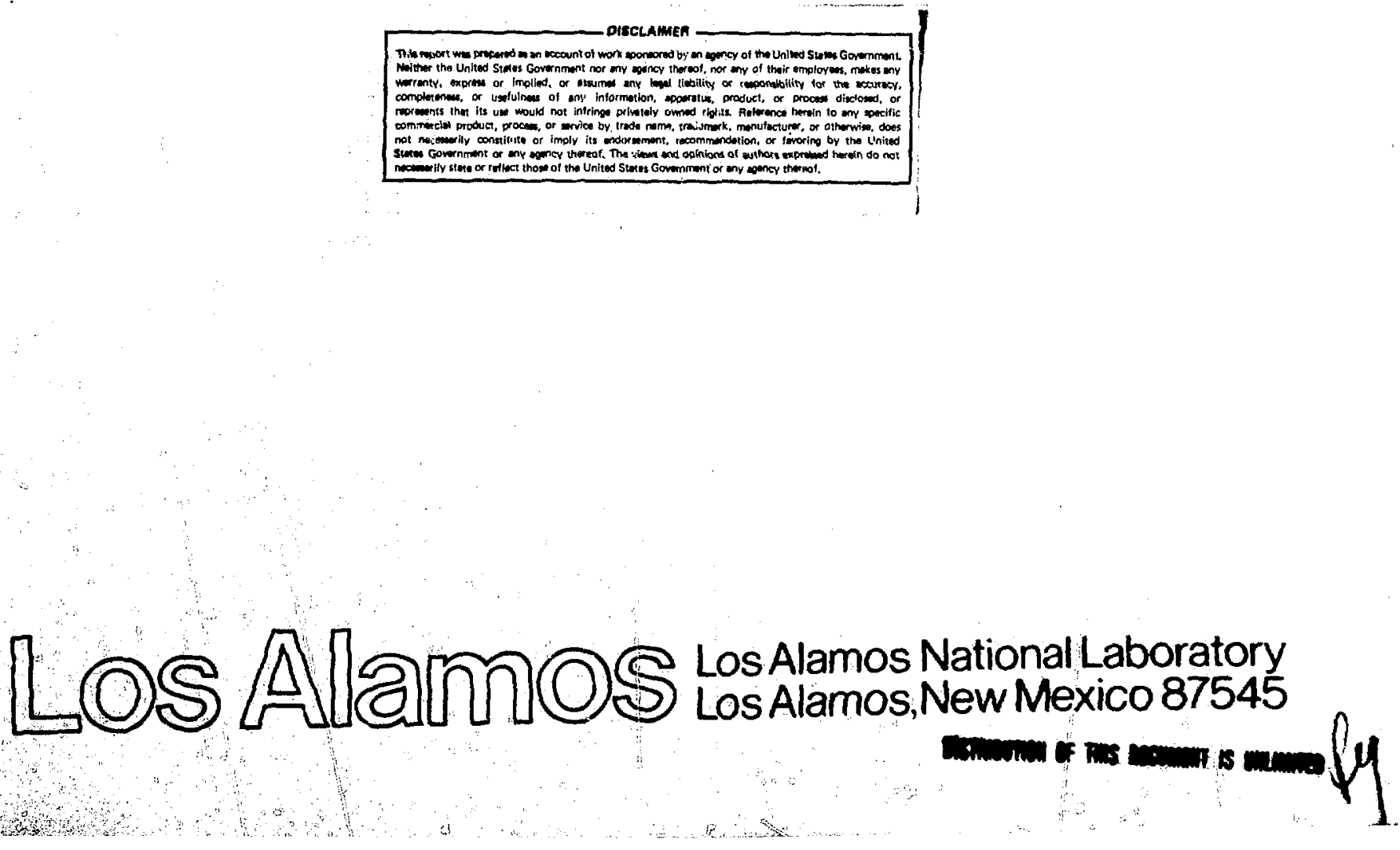


\section{APPLICATION OF ADJUSTED DATA IN CALCULATING FISSION-PRODUCT DECAY ENERGIES AND SPECTRA}

by

D. C. George, R. J. LaBauve, and T. R. England

\section{ABSTRACT}

The code ADENA, which approximately calculates fission-product beta and gamma decay energies and spectra in 19 or fewer energy groups from a mixture of ${ }^{235} \mathrm{U}$ and ${ }^{239} \mathrm{Pu}$ fuels, is described. The calculation uses aggregate, adjusted data derived from a combination of several experiments and sumation results based on the ENDF/B-V fission-product file. The method used to obtain these adjusted data and the method used by ADENA to calculate fission-product decay energy with an absorption correction are described, and an estimate of the uncertainty of the ADENA results is given.

Comparisons of this approximate method are made to experimental measurements, to the ANSI/ANS 5.11979 standard, and to other calculational methods. A listing of the complete computer code (ADENA) is contained in an appendix. Included in the listing are data statements containing the adjusted data in the form of parameters to be used in simple analytic functions. These fitted parameters can be abstracted for other uses such as in spatial neutron depletion or thermal hydraulics codes.

\section{INTRODUCTION}

Sumation calculations of fission product decay energy based on four different fission-product data files were compared with several experiments, 1,2,3,4,5 and the results were reported in Ref. 6. The conclusions dram from these comparisons include

(1) The experimental spectral data are consistent. 
(2) Aggregate beta and gamma spectral decay energies calculated from any of the four fission product files do not agree we11 with experiment for short irradiation and cooling times below $\sim 1000 \mathrm{~s}$.

(3) It is likely that some data in ENDF/B-V (Mod 0$)^{7}$, probably the experimental decay energies for some individual, high-Q nuclides, are deficient.

These conclusions imply that better estimates of decay spectra will result from calculations that use aggregate data derived, where possible, from experiments rather than data derived entirely from the fission-product files. However, because such experimental data are available only in the region $2.2 \mathrm{~s}-2 \mathrm{x}$ $10^{5} \mathrm{~s}$ for gamma decay energy and $2.2 \mathrm{~s}-10^{4} \mathrm{~s}$ for beta decay energy, information based on ENDF/B-V summation calculations was incorporated to extend the range of the calculated decay-energy cooling times from $10^{4}-10^{9} \mathrm{~s}$. The method used to prepare the adjusted data base is described in Section II.

The code ADENA uses these adjusted data to calculate fission-product decayenergy spectra from any mixture of thermally irradiated ${ }^{235} \mathrm{U}$ and ${ }^{239} \mathrm{Pu}$ fuels at user specified cooling times. A correction for neutron absorption in the fission products is included. Section III contains a description of the code, and Appendix A contains a full listing and a sample problem input and output. The spectra, but not the absorption correction, should be almost as accurate for the fissions induced by fast energy neutrons as it is for thermal neutrons.

Section IV contains the results of three applications of the code ADENA. First, the ADENA results for a finite irradiation problem including the effects of neutron absorption are compared with CINDER-10 (Ref. 8) calculations. Second, sample calculations of both the Oak Ridge and Los Alamos experiments are compared with the experiments. Third, a calculational comparison of the summed spectra with the ANSI/ANS-5.1-1979 standard 9 is made.

Finally, Section $V$ contains the authors' estimate of the reliability of ADENA results. Appendix $B$ clarifies the procedure used to obtain this estimate and includes in tabular form the detailed data upon which the estimate is based.

II. PREPARATION OF ADJUSTED DATA

The adjusted data base used by ADENA is derived from a combination of experimental data and the ENDF/B-V fission-product data file. The experiments, whose results were incorporated into the data base, were conducted at Los Alanos and Oak Ridge; nuclear fuel samples were irradiated with thernal neutrons and 
the decay-energy and beta-ray and/or ganma-ray spectra of the resulting fission products measured. Results of these experiments were included in formulating the ANSI/ANS-5.1 Decay Power Standard. A brief sumary of the experimental range of data follows.

- Oak Ridge spectral experiments ${ }^{1,2}$ in which ${ }^{235 \mathrm{U}}$ and ${ }^{239} \mathrm{Pu}$ fuels were irradiated with thermal neutrons for times of 1, 10, (5 for ${ }^{239} \mathrm{Pu}$ ), and $100 \mathrm{~s}$, and both aggregate fission-product gasma-ray and beta-ray decay-energy spectra were measured for a range of average cooling times from 2.2 (for the 1-s irradiation time) to $12000 \mathrm{~s}$ (for the 100-s irradiation time). There were similar measurements for ${ }^{241} \mathrm{Pu}$.

- Los Alamos calorimetric experiments ${ }^{394}$ in which ${ }^{233} \mathrm{U},{ }^{235} \mathrm{U}$, and ${ }^{239} \mathrm{Pu}$ were irradiated with thermal neutrons for $20000 \mathrm{~s}$ and total decay heat (gamma plus beta) measured for a range of cooling times from 29-190 $000 \mathrm{~s}$.

- Los Alamos spectral experiments ${ }^{5}$ in which fuels, irradiation time, and cooling time ranges were the same as for the calorimetric experiments, but aggregate fission-product gama-ray decay-energy spectra were measured.

The ENDF/B-V fission-product data file contains data for 877 nuclides, of which 264 have spectra, and there are 20 yield sets. These data for individual nuclides were input to the summation code CINDER-10,8 and the associated code system described in Refs. 10 and 11 was used to produce calculated decay energies and spectra. The aggregate experimental and sumation code results were combined to produce the adjusted data base using the procedure described below.

Step 1: Use $E N D F / B-V$ based summation results to calculate points from $10^{4}$ $s$ (beta) or $2 \times 10^{5} \mathrm{~s}$ (gamma) to $10^{9} \mathrm{~s}$ following a fission pulse. (As noted below, caclulations for shorter times were included in the detailed procedure.)

Step 2: Use the shape of the ENDF/B-V derived decay-energy curves below 2 s cooling time shifted to coincide with the experimental data having the shortest cooling times to calculate points below $2 \mathrm{~s}$.

Step 3: Combine points from steps one and two with experimental data points. Use this set of combined points as input to FITPUis ${ }^{11}$ to calculate a set of paranetric pairs, which represents a fit for a combined adjusted equivalent pulse.

A more detailed description of the procedure outlined in these three steps follows. 


\section{ENDF/B-V Calculation - Preliminary}

Methods described in Refs. 10 and 11 were used to create sets of alpha $(\alpha)$, lambda $(\lambda)$ parameter pairs which represent least squares fits to the aggregate ENDF/B-V pulse data by fitting the data with the equation,

$$
f(t)=\sum_{i=1}^{n} \alpha_{i} e^{-\lambda_{i} t} \quad(\text { MeV/fis-s) }
$$

These sets of parameter pairs were obtained for beta- and gama-ray decay, for both ${ }^{235} \mathrm{U}$ and ${ }^{239} \mathrm{Pu}$ fuels, for all decay-energy groups, over the full cooling time range of $10^{-4}-10^{9} \mathrm{~s}$. The generation of these sets was a necessary preliminary step to obtaining the adjusted data base, and the sets are used in each of the three steps previously outlined.

Long Cooling Times - Step 1

The sets of parameters from the ENDF/B-V calculation were used in Eq. (1) to compute the beta- and gamma-ray decay energy at four cooling times per decade from the longest experimental cooling time $\left(10^{4} \mathrm{~s}\right.$ for beta-ray decay and $2 \times 10^{5} \mathrm{~s}$ for gamma-ray decay) up to $10^{9} \mathrm{~s}$ for each decay energy group for both fuels. Because of large experimental errors, ENDF/B-V data were used to calculate points from $10^{3}-10^{9} \mathrm{~s}$ for some of the higher energy groups.

Cooling Times Less Than Two Seconds - Step 2

Because there is often a large difference between the decay energy for short cooling-times calculated from the ENDF/B-V fits and the experimentally measured values, we used the shape of the ENDF/B-V data shifted to coincide with the equivalent experimental short cooling time points. [The derivation of "equivalent experimental points" (pulse values) is discussed below.] The shift was accomplished by first plotting the ENDF/B-V fit and the equivalent experimental pulse points having a cooling time less than $10 \mathrm{~s}$. Figure 1 is an example of one of these plots. The ENDF/B-V curve was then shifted manually to coincide with the equivalent experimental points, and values for cooling times of $0.01,0.03,0.1,0.3$, and 1.0 seconds were read off the graph. (Figure 2 shows the resulting adjusted fit for cooling times less than 10 s after the procedures described in steps 1-3 have been completed.)

It is important to note that the equivalent experimental points plotted on Figs. 1-3 are produced from the original data in the following manner. The original experimental points are measured beta- or gama-ray decay energies at cooling tines of $t$ seconds after an irradiation period of $T$ seconds. In order 
Fig. 1. Beta-ray fission-product decay energy for Group 5 (0.6-0.8 MeV) calculated from ENDF/B-V data and "equivalent pulse" experimental points.
Fig. 2. Beta-ray fission-product decay energy for Group 5 (0.6-0.8 MeV). Adjusted curve shows the result of shifting the ENDF/B-V curve to coincide with the "equivalent pulse" experimental points.
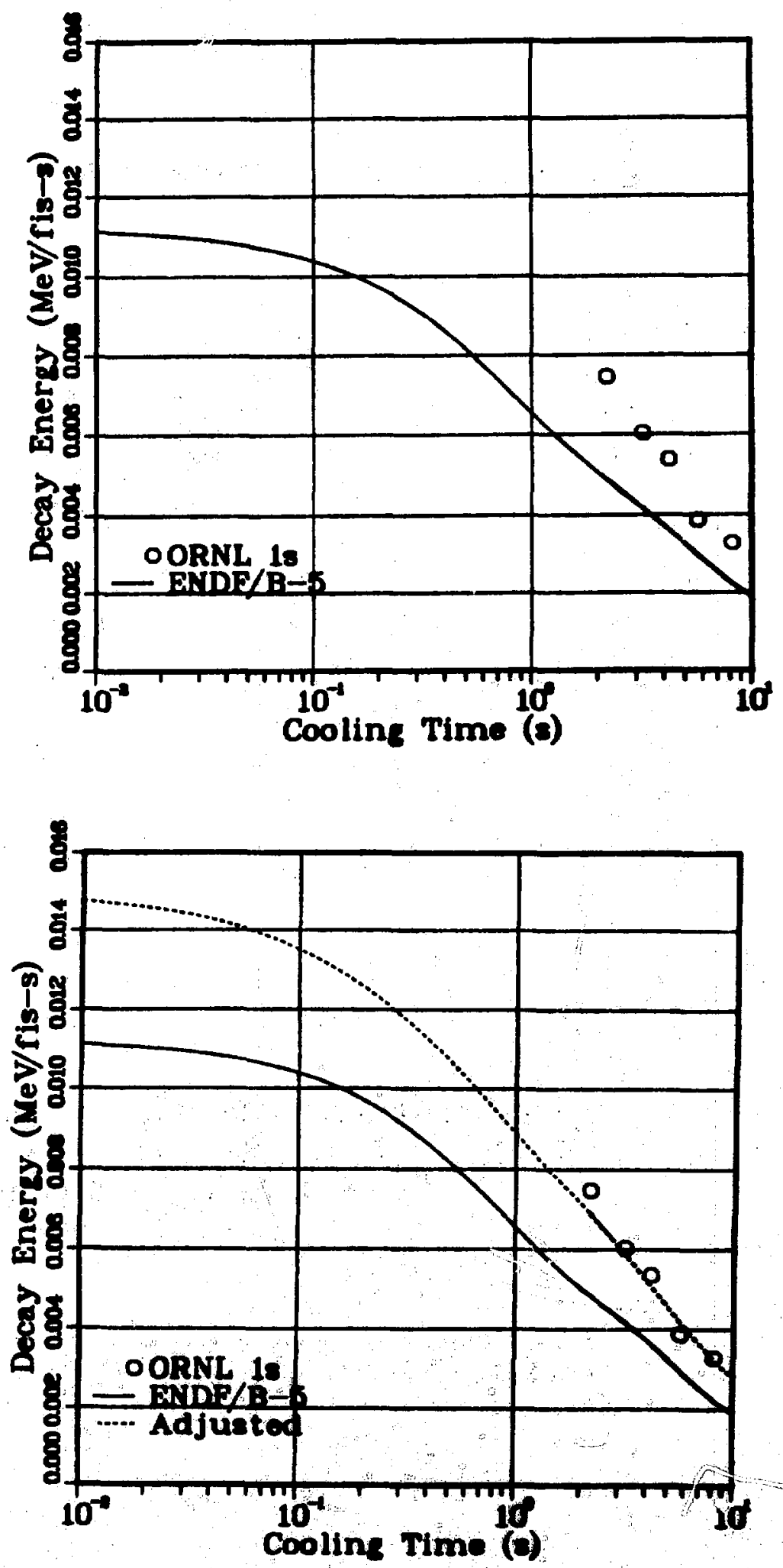


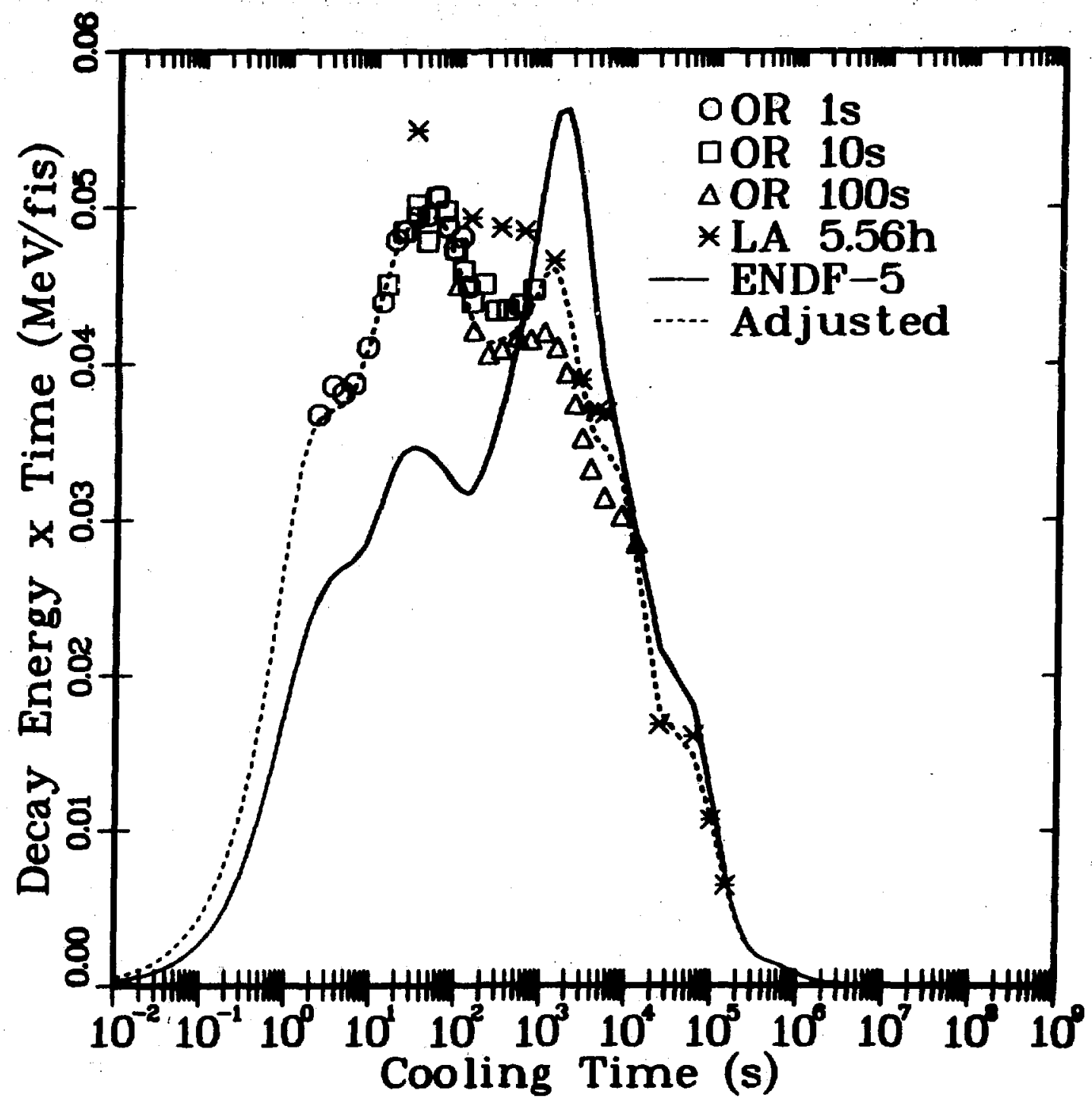

Fig. 3. Gamma-ray decay energy for Group 7 (1.0-1.2 MeV) showing "equivalent pulse" experinental points, ENDF/B-V fit, and adjusted fit. 
to intercompare experiments with different irradiation times and to compare these experiments to calculations, it is desirable to reduce the experimental data to equivalent pulse data that are independent of irradiation time. The code FITPULS can generate a set of alphas and lanbdas by fitting the original experimental data with an integration of $\mathrm{Eq}$. (1) over $\mathrm{T}$ for a unit fission rate producing functions of the form,

$$
F(T, t)=\sum_{i=1}^{n} \frac{\alpha_{i}}{\lambda_{i}} e^{-\lambda_{i} t}\left(1-e^{-\lambda_{i} T}\right)(\text { MeV/fis })
$$

The set of alpha, lambda parameter pairs derived by FITPULS using Eq. (2) fron the combination of all experimental data points constitute the experimental pulse fit. See Appendix D of Ref. 10 for a detailed treatment of this subject. During this fitting process, the percent differences of the original experimental data points to the fitted results as calculated by Eq. (2) are computed and saved. These percent differences can be applied to the experimental pulse by evaluating Eq. (1) at the cooling times of the original experimental points and adding the percent differences to generate equivalent experimental pulse data points. Because the fitting procedure involves a nonlinear least squares algorithm, neither the experimental pulse fits nor the equivalent experimental pulse points are unique. For graphical comparisons, the equivalent experimental pulse values are an excellent representation of the experiments because deviations are emphasized. The actual fitting process of step 3 (below) uses the original experimental data points, not the equivalent points.

Final Paraneters Representing the Adjusted Data - step 3

Points from Step 1 computed directly using the wnF/BV pulse parameters in Eq. (1) for long cooling times, points from step 2 derived from the shifted ENDF/BV pulse fit for short cooling times, and the original experimental data points for the middle cooling time region were combined. The combination was input along, with the ENDF/BV pulse paraneters to FITPULS, which produced set: of alpha, lambda paraneter pairs constituting the adjusted equivalent pulse fits. FITPULS uses a nonlinear least squares procedure to fit the input to sq. (1) (for the pulse points from Steps 1 and 2) and Eq. (2) (for the original experimental finite-irradiation data pointi) using the WDF/D-V pulae perameters as the starting values for the fitting procese. Thus, the adjucted 
fits reflect the basic shape of the ENDF fit, as can be seen in Fig. 3, which shows the original ENDF fit, the final adjusted fit, and the equivalent pulse experimental data points for each experinent. The fitting procedure allows for weights to be assigned to the data points (experimental data or points created by the methods described in Steps 1 and 2). By looking at graphs of the equivalent experimental points and the ENDF fits, it can be determined which points should be given light weights and which should be given heavy weights. Heavy weights will force the final fit to adhere closely to those points, whereas light weights will allow the fit to wander quite far from the data points.

These adjusted fits were obtained in the 19-energy group structure given in Table $I$ for ${ }^{235} \mathrm{U}$ beta- and gamma-ray decay-energy spectra and for ${ }^{239} \mathrm{Pu}$ beta- and gamma-ray decay-energy spectra. Note that for cooling times outside the range of the experiments, only ENDF/B-V data were used for FITPULS input; thus the adjusted fits for cooling times greater than $10^{4} \mathrm{~s}$ for betas and $2 \times 10^{5}$ s for gammas are just fits to the calculated ENDF/B-V sumation data. Because the experimental error at cooling times greater than 1000 s for energies greater than $4 \mathrm{MeV}$ is very large for groups 17 and 18 , those experinental points were ignored and the ENDF/B-V data were used instead. Thus, for these groups the adjusted fits are the ENDF/B-V fits for cooling times greater than $1000 \mathrm{~s}$. There are no experimental data for group 19, therefore the adjusted fit for group 19 is based on the ENDF/B-V fit for all cooling times.

\section{DESCRIPTION OF ADENA}

The program ADENA was written to approximate the fission product decayenergy spectra with an absorption correction for fuel wixtures of ${ }^{235} U$ and ${ }^{23} \mathrm{Pu}$. The adjusted fits produced by the procedure given in Sec. II were incorporated into the code, which uses these paraneters in Eq. (2) to calculate the fission product decay energy for a finite irradiation tine without absorption. A description of the input to ADENA is given in Table II.

For many applications involving long irradiation times, a correction to the adjusted fits is needed to account for the effects of neutron absorption. (Appendix $D$ of Ref. 10 gives the general equations to calculate absorption effects; however, the simplified nethod developed in Ref. 12 was used in ADENA.) Several liniting assumptions are made in order to simplify the absorption calculations. The power history must be reduced to the associated 


\section{TABLE I}

\section{ENERGY GROUP STRUCTURE}

\begin{tabular}{ccc} 
Group & E-Lo(HeV) & E-Hi(MeV) \\
\cline { 2 - 3 } 1 & 0.0 & 0.1 \\
$2 *$ & 0.1 & 0.2 \\
3 & 0.2 & 0.4 \\
4 & 0.4 & 0.6 \\
5 & 0.6 & 0.8 \\
6 & 0.8 & 1.0 \\
7 & 1.0 & 1.2 \\
8 & 1.2 & 1.4 \\
9 & 1.4 & 1.6 \\
10 & 1.6 & 1.8 \\
11 & 1.8 & 2.0 \\
12 & 2.0 & 2.2 \\
13 & 2.2 & 2.4 \\
14 & 2.4 & 2.6 \\
15 & 2.6 & 3.0 \\
16 & 3.0 & 4.0 \\
17 & 4.0 & 5.0 \\
18 & 5.0 & 6.0 \\
19 & 6.0 & 7.5
\end{tabular}

\footnotetext{
* Due to lack of experimental data, Groups 1 and 2 were combined for the beta-ray calculations.
}

average thermal and epithermal fluxes. The requested group structure must be a subset of the group structure given in Table $I$. Only the two most important chains ${ }^{155} \mathrm{Eu}-{ }^{156} \mathrm{Eu}$ and ${ }^{133} \mathrm{Cs}_{\mathrm{B}}-{ }^{134} \mathrm{C}_{\mathrm{s}}$ are considered; these have a net positive effect on heating and spectra." The correction is given by the equation

$$
\Delta F(t, T, \phi)=\Delta W(T, \phi) \lambda w e^{-\lambda t}
$$

where,

$T$ is the irradiation tine

$t$ is the cooling time

1 is the neutron flux (thermal and epithermal)

$\lambda$ is the decay constant of the second nuclide in the chain

$w$ is the average photon decay energy for a given group for the second nuclide

$\Delta \mathrm{N}(\mathrm{T}, \phi)$ is the change in aton density of the nuclide resulting fro its radiative capture and capture in its precureor. 


\section{ADENA INPUT SPECIFICATIONS}

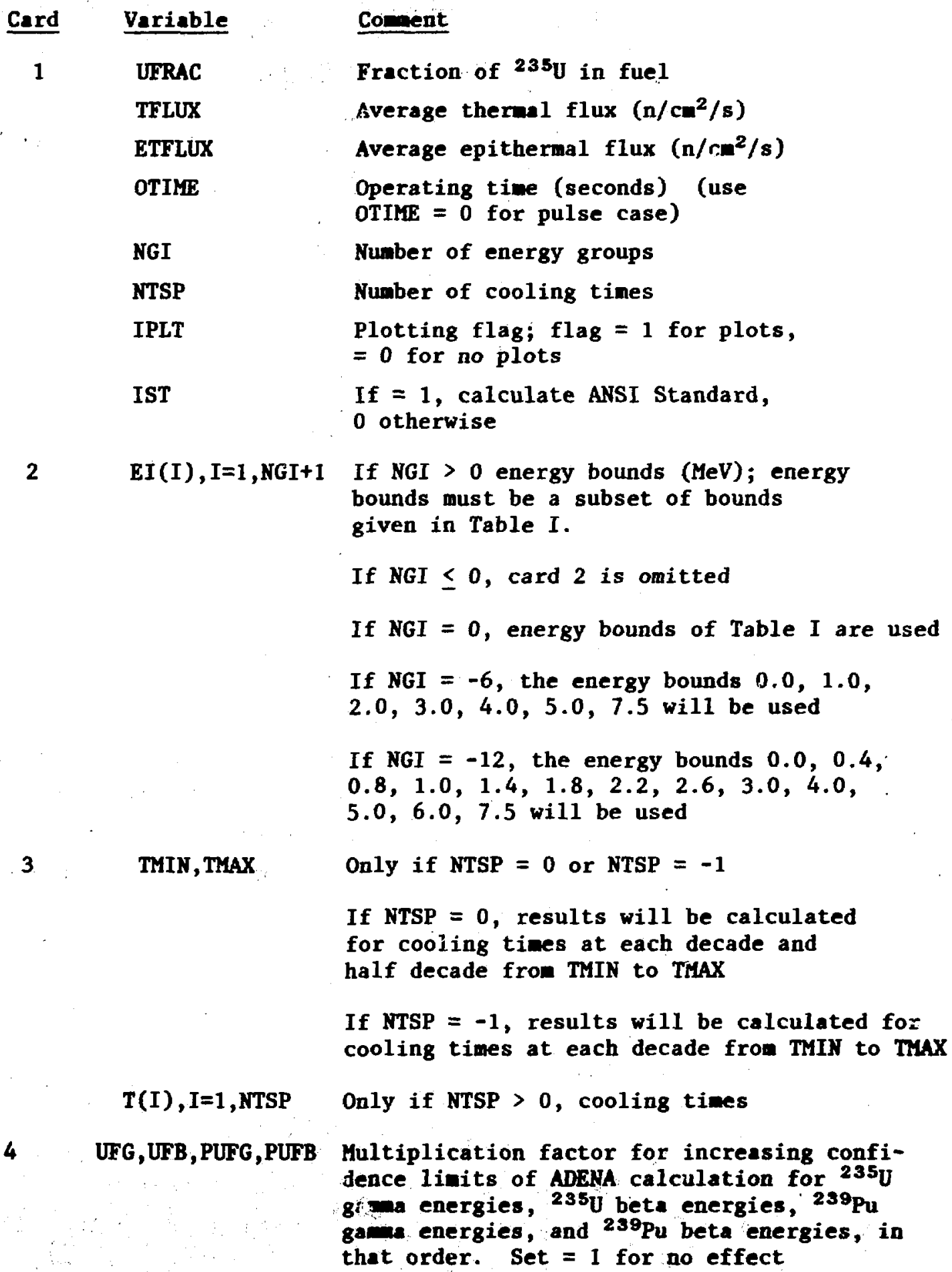

A11 Input is free field; values or comms must be supplied for all variables. 
Results are generated for each requested cooling tine by group and for the sum over all groups. Beta-ray, gama-ray, and the su of beta- and gama-ray decay energies are printed and plotted for each category.

The ADENA code also has "the capability of calculating the decay energy after a pulse (specify OTIME $=0$ ) and of performing calculations of the total beta plus gama decay energy based on the ANSI/ANS-5.1-1979 Standard (specify IST $=1$ ), as described in Table II.

\section{COMPARISONS}

To check the data fits used in ADENA, three types of comparisons were made. The first comparison involved using ADENA to calculate the gamaray decay energy at three cooling times $\left(1,10^{6}\right.$, and $10^{8}$ s) for a ${ }^{235} \mathrm{U}$-fueled thermal reactor with an average thermal $f l u x$ of $10^{14} \mathrm{n} / \mathrm{cm}^{2} / \mathrm{s}$ for an operatins time of 20000 hours. The ADENA results were then compared with the results of a CINDER-10 (Ref. 8) calculation of the sane problea. The ADENA calculation used the adjusted data base derived from experiment and ENDF/B-V, whereas the CINDER calculation used ENDF/B-IV data. The results of both calculations are given in Table III. The biggest differences occur at the shortest coolins time, $1 \mathrm{~s}$, in the lowest energy group. This observation is supported by the results of the data testing study ${ }^{6}$ that indicates that the calculated gana-ray decay energies are relatively high for early cooling times and sall gama-ray energies. Maximum absorption effects in the calculations are at $10^{6}$ cooling time for the europium chain and $10^{8} \mathrm{~s}$ for the cesium chain. As can be seen from the tabulations in Table III, ADENA agrees well with CINDER at these times.

The second type of comparison involved using ADENA to calculate the 0ak Ridge (ORIL) and LOS Alamos experiments, and plotting the calculation with the experimental data. Examples of these graphical comparisons for the 0an 100-s irradiation experiment ${ }^{1}$ are given in Figs. 4-6 for three coolins tines (90, 950, and $11950 \mathrm{~s}$ ) and for the Los Alawos 5.56-h experiment in Figs. 7-9 for three cooling times (128, 1218, and $14650 \mathrm{~s})$. As can be seen in the figures, the calculation agrees quite well with the experiment. Wote that for loas cooling times and high gave-ray energies, the experimental error is very large.

A finsi comparison is ade with the $1979 \mathrm{NSI} / \mathrm{Ns}-5.1$ bealderd. This atandard is believed to provide the best estifite of total (beth plas oud) 
TABLE III

COMPARISON OF GAMMA-RAY ENERGY CALCULATED BY ADENA AND CINDER

\begin{tabular}{|c|c|c|c|c|c|c|c|}
\hline \multirow[b]{2}{*}{$\begin{array}{c}\text { Group } \\
\\
1 \\
2 \\
3 \\
4 \\
5 \\
6\end{array}$} & $\begin{array}{c}\text { Energy Bounds } \\
\text { (MeV) }\end{array}$ & \multicolumn{2}{|c|}{$\begin{array}{c}\text { Cooling Tine } 1 \text { s } \\
\text { Gana Decay Energy } \\
\text { (HeV/fis) }\end{array}$} & \multicolumn{2}{|c|}{$\begin{array}{c}\text { Cooling Tine } 10^{6} \mathrm{~s} \\
\text { Gamm Decay Energy } \\
\text { (HeV/fis) }\end{array}$} & \multicolumn{2}{|c|}{$\begin{array}{c}\text { Cooling Time } 10^{8} \mathrm{~s} \\
\text { Gawa Decay Energy } \\
\text { (MeV/fis) }\end{array}$} \\
\hline & $\begin{array}{l}0.0-1.0 \\
1.0-2.0 \\
2.0-3.0 \\
3.0-4.0 \\
4.0-5.0 \\
5.0-6.0\end{array}$ & $\begin{array}{l}\text { CINDER } \\
2.681 \\
2.061 \\
0.838 \\
0.306 \\
0.166 \\
0.042\end{array}$ & $\begin{array}{l}\text { ADENA } \\
2.258 \\
1.971 \\
0.968 \\
0.421 \\
0.179 \\
0.057\end{array}$ & $\begin{array}{l}\text { CINDER } \\
0.180 \\
0.073 \\
0.008 \\
0.001 \\
0.001 \\
0.000\end{array}$ & $\begin{array}{l}\text { ADENA } \\
0.181 \\
0.067 \\
0.009 \\
0.000 \\
0.000 \\
0.000\end{array}$ & $\begin{array}{l}\text { CINDER } \\
0.0079 \\
0.0003 \\
0.0001 \\
0.0000 \\
0.0000 \\
0.0000\end{array}$ & $\begin{array}{l}\text { ADENA } \\
0.0077 \\
0.0003 \\
0.0000 \\
0.0000 \\
0.0000 \\
0.0000\end{array}$ \\
\hline Total & $\because$ & 6.095 & 5.856 & 0.261 & 0.257 & 0.0083 & 0.0081 \\
\hline $\begin{array}{l}\text { Fuel } \\
\text { Therma } \\
\text { Epithe } \\
\text { Operat }\end{array}$ & $\begin{array}{ll}235 \mathrm{y} \\
\text { Flux } & 10^{14} \\
\text { cmal Flux } & 5 \times \\
\text { ing Time } & 200\end{array}$ & $\begin{array}{l}/ \mathrm{cm}^{2} / \mathrm{s} \\
1 \mathrm{~s}_{\mathrm{hr}} \mathrm{n} / \mathrm{cm}^{2}\end{array}$ & & & & & \\
\hline
\end{tabular}

decay heating, but it does not provide a resolution into the beta and gama components or spectra. The primary intent of this report is to provide a simple code that will calculate our best estimate of these components, particularly their spectra on a few-energy group besis. The total heating closely matches values from the standard, as shown in Fig. 10 for $235 \mathrm{~J}$ and Fig. 11 for ${ }^{239} \mathrm{Pu}$, but is not normalized to the standard. The ADENA results are within $10 \%$ of the standard at all times. In terms of the small assigned uncertainties of the standard, the ADENA results are all within a two-sigma uncertainty of the standard for ${ }^{230} \mathrm{Pu}$. The uncertainties in the standard are much smaller for $235 \mathrm{U}(<2 \%)$, and 14 of the 46 points are further than 2 sigma from the standara (a11 are within 10\%), 4 points are further than 3 sigma, and all are within 4 sigma uncertainty of the standard. Note that the standard is based on five experiments and ENDF/B-IV data, whereas our adjusted fits are based on only three experiments and $E N D F / B-V$ data.

v. ESTIMATE OF ACCURACY OF CALCULATIONS USING THE ADENA CODE

As described in Sec. III, the user has the option of assigning values to four input parameters in ADENA, namely, UFG, UFB, RUFG, and PUFB, which will raise or lower the results of the calculation by a certain percentage and thus increase the level of confifsce in the calculation. It is the intent of this 
F1g. 4. Gamma-ray decay energy after a cooling time of $90 \mathrm{~s}$ from the Oak Ridge National Laboratory 235U 100-s irradiation experiment compared with the ADENA calculation.

Fig. 5. Gamma-ray decay energy after a cooling time of $950 \mathrm{~s}$ from the Oak Ridge National Laboratory $235 \mathrm{U}$ 100-s irradiation experiment compared with the ADENA calculation.
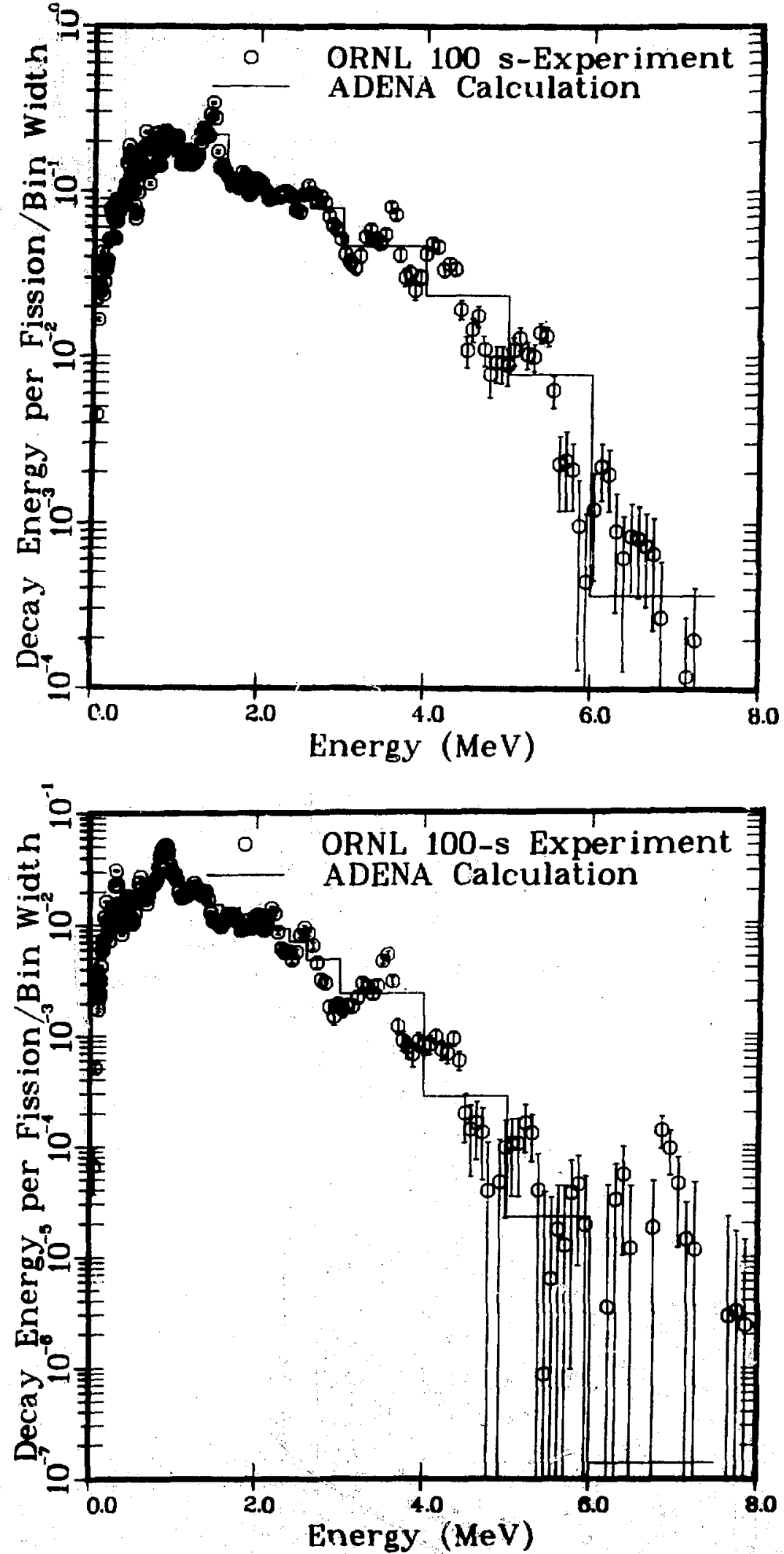
F1g. 6. Gamma-ray decay energy after a cooling time of $11950 \mathrm{~s}$ from the Oak Ridge National Laboratory 235 U 100-a 1rradiation experiment compared with the ADENA calculation.
Fig. 7. Gamma-ray decay energy after a cooling time $8 f_{5} 128$ s from the Los Alamos $235_{\mathrm{U}}$ 5.56-h irradiation experiment compared to the ADENA calculation.
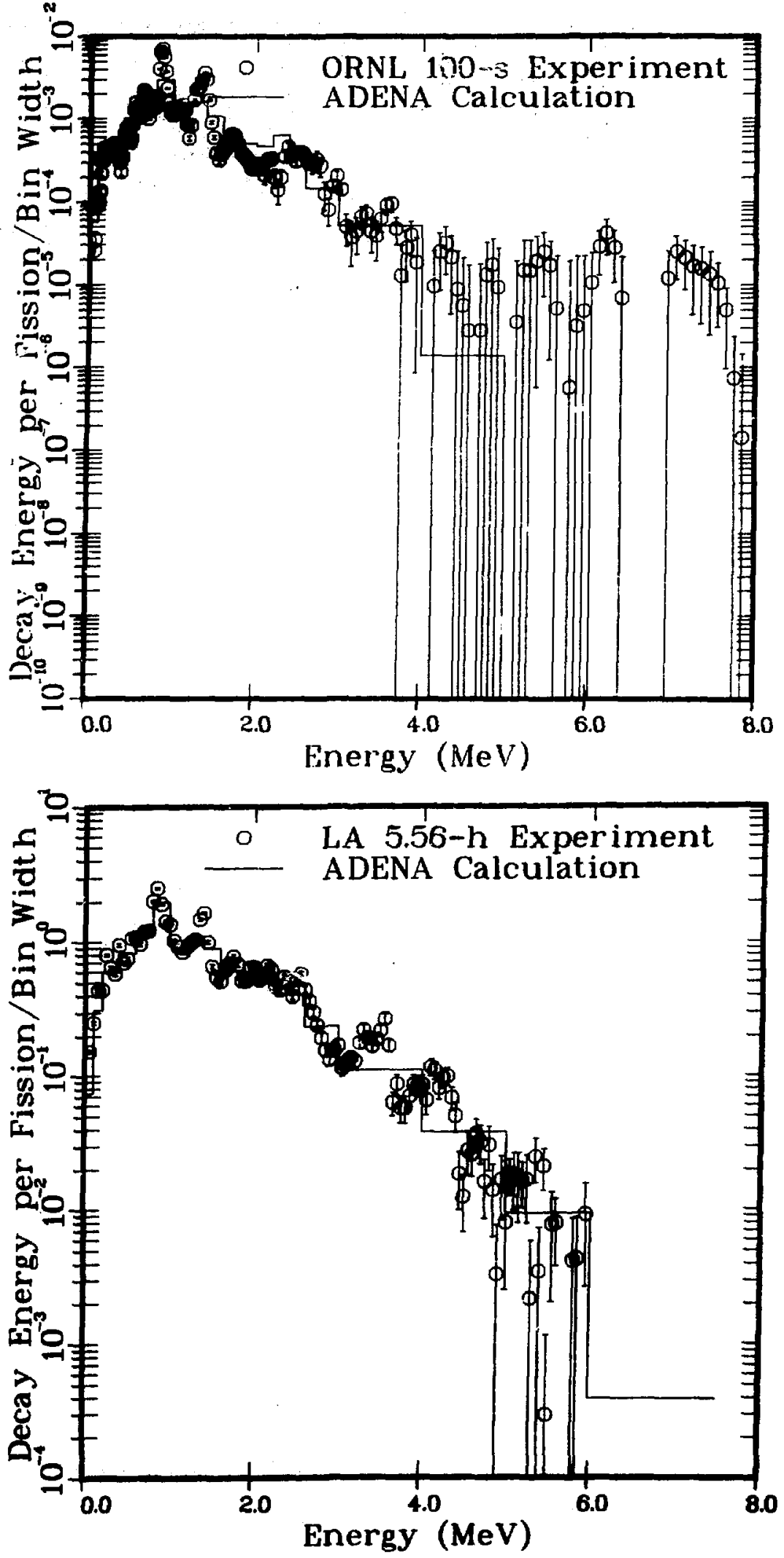
Fig. 8. Gamma-ray decay energy after a cooling time of $1218 \mathrm{~s}$ from the Los Alamos 235U 5.56-h irradiation experiment compared to the ADENA calculation.

Fig. 9, Gamma-ray decay energy after a cooling time of $14650 \mathrm{~s}$ from the Los Alamos 235U 5.56-h irradiation experiment compared to the ADENA calculation.
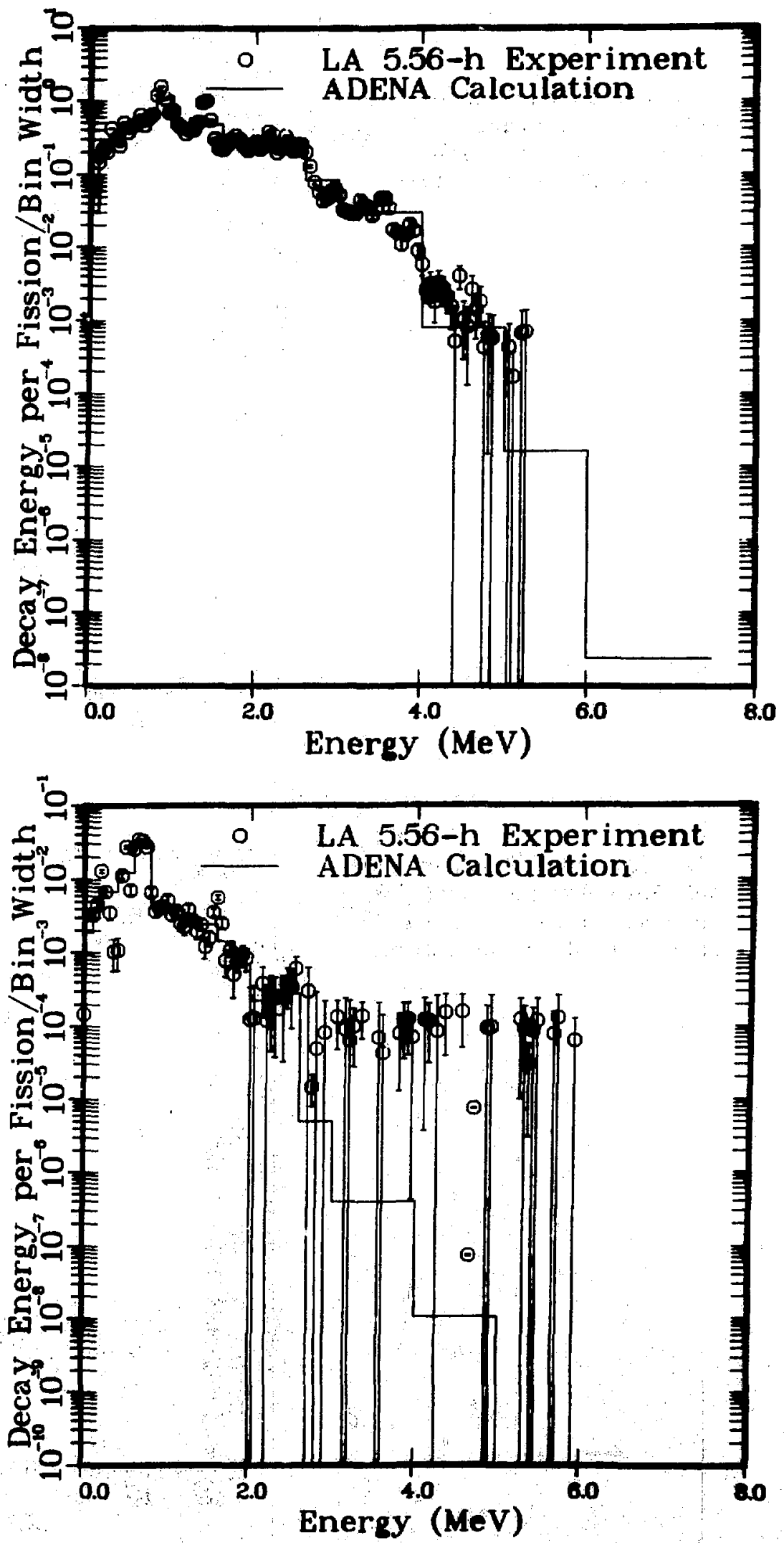
F1g. 10. Comparison of ANSI/ ANS-5.1-1979 standard for 235 therwal pulte fisaton of 235 to the ADEX calculation.

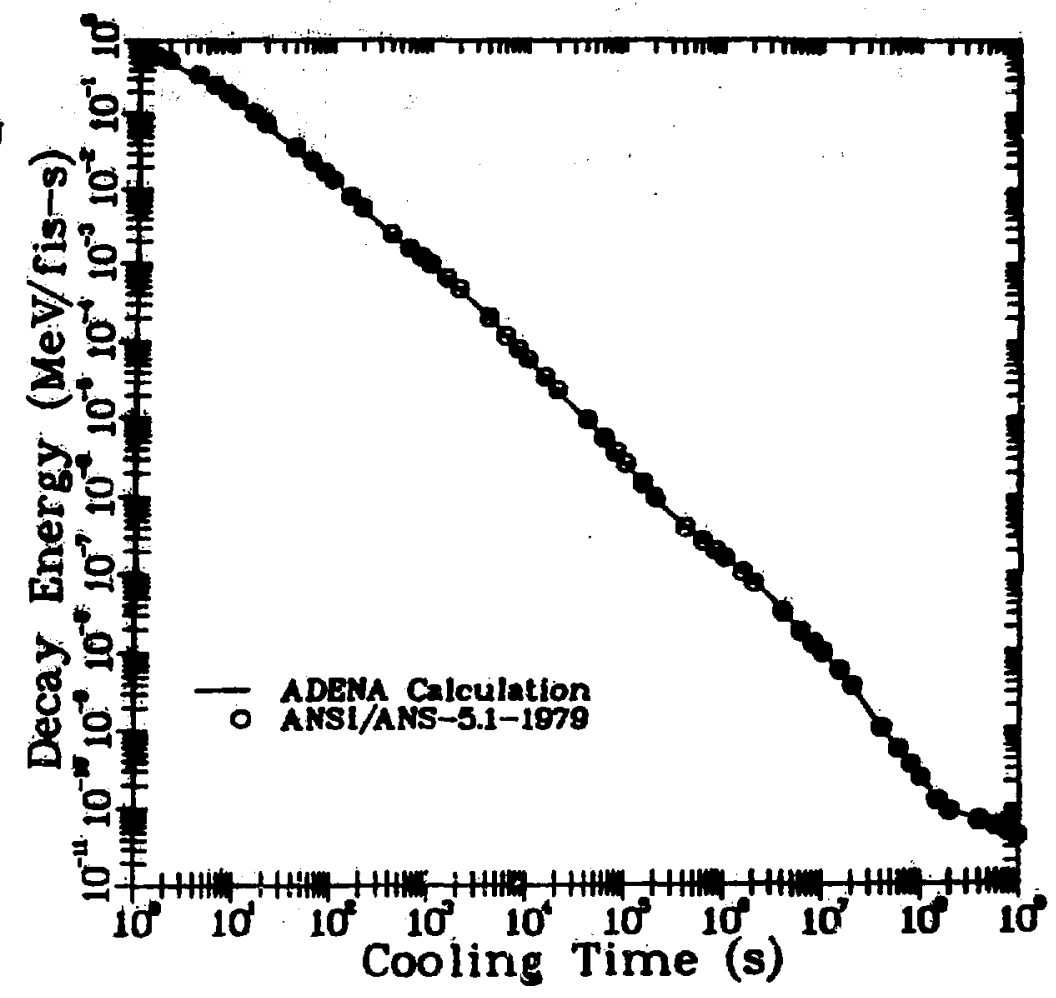

Fig, 11, Comparison of ANST/ ANS $-5.1-1979$ standard for
thermal pulse fission of ${ }^{2} \mathrm{Pu}$ to the ADENA calculation.

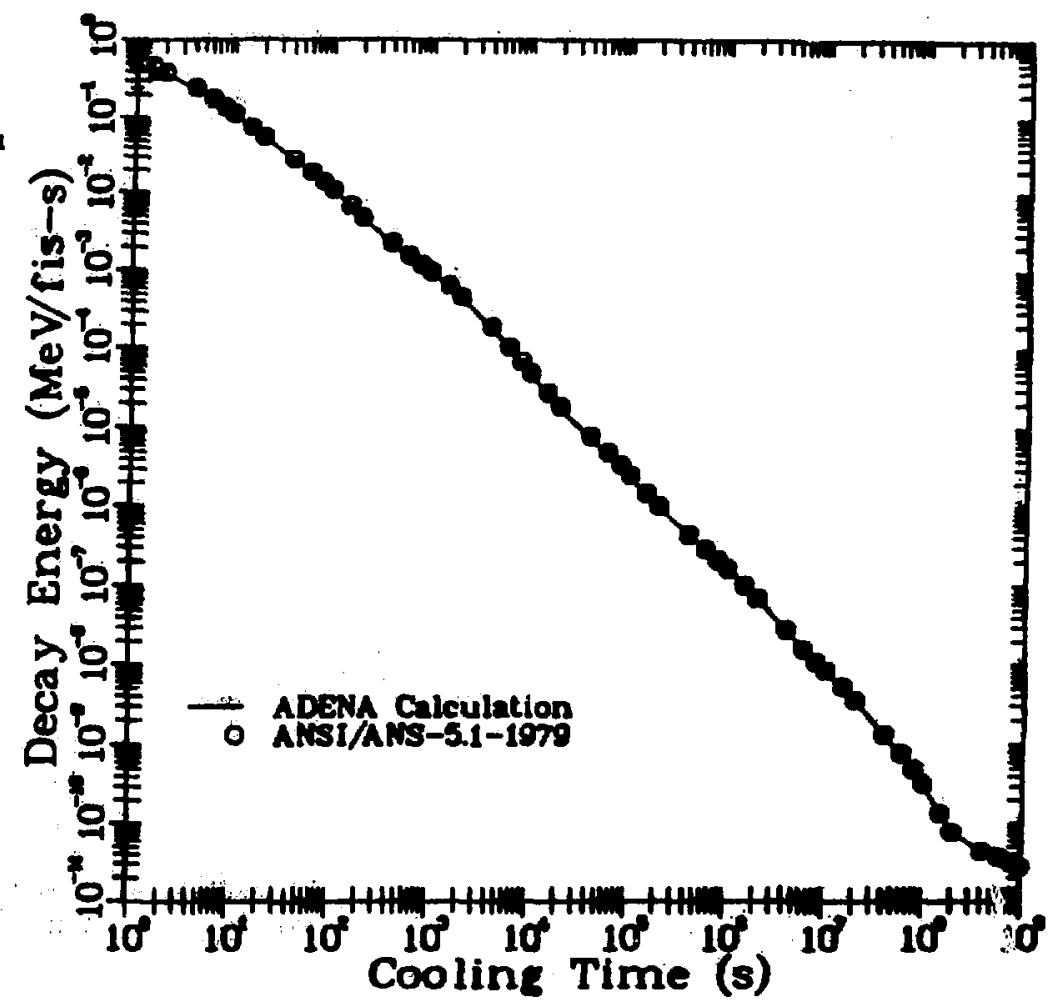


section to provide the user with some guidance in assigning values to these parameters. Overall reliability of calculations with ADENA depends upon (a) the accuracy of the adjusted spectral fits and (b) the accuracy of the neutron absorption approximation. Some indication of the accuracy of the fits is obtained by the calculation of the standard noted in Sec. IV. Note, however, that this is for the decay energy fros combined beta- and gama-ray decay for the aggregate of the fission products and sumed over all. energy groups.

Results given in Sec. IV show that the values calculated by ADENA are all within the 2-sigma error quoted for the ${ }^{239} \mathrm{Pu}$ standard, but that 14 were outside the small 2-signa error quoted for $235 \mathrm{u}$. These 14 values are shown as a function of cooling time in Table IV. Note that 9 of the 14 values occur for cooling times of $100 \mathrm{~s}$ or less. We therefore suggest that for problems involving totals over energy (i.e., nonspectral) and total beta- plus gama-decay energy, an average uncertainty value of $7.5 \%$ be assuned for cooling times of $100 \mathrm{~s}$ or less, and an average of $4.0 \%$ be assuned for longer cooling tines. Uncertainties assigned for spectral calculations using the adjusted fits are considerably greater, however, and are more dependent on cooling-tine ranges as well as being dependent upon spectral energy ranges. A rather detailed discussion of the deviations of calculations with the adjusted fits from aggregate values from sumation calculations using ENDF/B-V and the deviations of adjusted fit calculations from experimental data are given in Appendix $B$. On the basis of the results of the analysis in this appendix, we recomend that the uncertainties given in Table $V$ be used over the energy and cooling-tine ranges given in the table for both beta- and gama-ray decay energies and for both ${ }^{235} \mathrm{U}$ and ${ }^{239} \mathrm{Pu}$ fuels. We further suggest that the user can easily modify this table according to his needs by referring to Appendix $B$.

Finally, Table III is a good example of the accuracy of the two-chain approximation that the ADENA code uses to calculate neutron absorption effects for cooling times above $10^{5} \mathrm{~s}$. As can be seen from the table, deviations from the sumation calculations do not exceed $2 \%$ for those cooling-time ranges where the absorption correction is significant. For shorter cooling tines. uncertainties in spectral values calculated with the adjusted fits are considerably greater than the deviation due to absorption effects. Consequently, as arule of thumb," we suggest that a minimu uncertainty of $10 \%$ be assigned in Table $V$ for ADENA calculations with significant absorption effect, $i$.e., large fluence and long cooling times. Neutron absorption is discussed ore fully in Refs. 10 and 12 . 
TABIE IV

ADENA CaLCULATIONS OUTSIDE 2-SIGMA OF ${ }^{235} \mathrm{U}$ STANDARD

$\begin{array}{ccc}\begin{array}{c}\text { Cooling Time } \\ (\mathrm{s})\end{array} & \begin{array}{c}\text { 1-Sigma Uncertainty } \\ \text { in Standard }(\boldsymbol{q})\end{array} & \begin{array}{c}\text { Calculation fron } \\ \text { Standard }(\boldsymbol{)})\end{array} \\ 4.0 \mathrm{E}+00 & 4.3 & 10.0 \\ 6.0 \mathrm{E}+00 & 3.5 & 8.9 \\ 8.0 \mathrm{E}+00 & 3.1 & 7.6 \\ 1.0 \mathrm{E}+01 & 3.2 & 6.7 \\ 2.0 \mathrm{E}+01 & 2.4 & 6.3 \\ 4.0 \mathrm{E}+01 & 2.0 & 8.1 \\ 6.0 \mathrm{E}+01 & 1.9 & 7.5 \\ 8.0 \mathrm{E}+01 & 1.9 & 6.5 \\ 1.0 \mathrm{E}+02 & 1.8 & 5.7 \\ 2.0 \mathrm{E}+02 & 1.9 & 4.8 \\ 4.0 \mathrm{E}+02 & 1.9 & 4.5 \\ 2.0 \mathrm{E}+03 & 1.8 & 3.9 \\ 2.0 \mathrm{E}+04 & 1.4 & 4.2 \\ 4.0 \mathrm{E}+04 & 1.4 & 3.3\end{array}$

TABLE $v$

PERCENT ACCURACY OF ADJUSTED SPECTRAL FITS

Energy

Ranges

(MeV).

$0.0-0.6$

$0.6-1.6$

$1.6-3.0$

$3.0-7.5$
Cooling-Time Ranges (s)

$1.0 \mathrm{E}-02-1.0 \mathrm{E}+00 \quad 1.0 \mathrm{E}+00-1.0 \mathrm{E}+04 \quad 1.0 \mathrm{E}+04-1.0 \mathrm{E}+06 \quad 1.0 \mathrm{E}+06-1.0 \mathrm{E}+09$

22.8

6.9

15.3

5.8

16.5

40.5
8.2

8.4

17.0

7.8
5.0

5.0

5.0

5.0

VI. SUMMARY

$A$ wethod for creating an adjusted fission-product decay-energy data base from combination of experimental data and the ENDF/B-V fission-product data file has been described. The code ADENA, which uses the adjusted data base in calculating fission-product decay-energy spectra (including the najor effects of neutron absorption) for fuel wixtures of ${ }^{235} \mathrm{U}$ and ${ }^{239} \mathrm{Pu}$, has also been described. The code can be used for a wide variety of reactor operational and safety related computations where aggregate fission-product decay spectra are needed. This avoids the need for the large data base and code systens ${ }^{10-12}$ we have used to produce this end product, assuming the user reguires only aggregate, 
rather than individual nuclide, results. We have, in addition, incorporated the important experimental spectra svailable for short cooling times.

Several examples of the application of the ADENA code are also given, including comparison to the ANSI/ANS 5.1-1979 Standard. A section is included from which the user can obtain an indication of the reliability of ADENA calculational results.

The adjusted parameters can be abstracted from the code listing and used directly in Eqs. (1) or (2) or the more general equations in the appendix of Ref. 10 by those users requiring spectral calculations in various spatial codes.

\section{ACKMOWLEDGMENTS}

For two fuels, the pulse functions described in this report and the associated ADENA code are the culmination of a series of related reports and codes. The need for and general utility of such functions was originally suggested by J. Lewellen and P. Hemigig (Department of Energy). Along the way, we have enjoyed discussions contributing to our work with $R$. Schenter, F. Schnittroth, and F. Mann (Hanford Engineering Development Lab); A. Tobias and colleagues (Central Electricity Board, U.K.); and T. Yoshida and colleagues (Nippon Atomic Industry Group Nuclear Research Laboratory, Japan). The experimental data provided by J. K. Dickens (Oak Ridge), and J. Yarnell and E. Jurney (Los Alamos) contributed vitally to this work and to earlier comparisons.

\section{REFERENCES}

1. J. K. Dickens, T. A. Love, J. W. McConnell, J. F. Emery, K. J. Northcutt, and R. W. Peelle, "Delayed Bet ${ }_{\omega}^{-}$and Gama-Ray Production Due to ThermalNeutron Fission of ${ }^{235} \mathrm{U}$, Spectral Distributions for Tines After Fission Between 2 and 14000 sec: Tabular and Graphical Data," Oak Ridge National Laboratory report NUREG/CR-0162, ORNL/NUREG-39 (August 1978).

2. J. K. Dickens, T. R. England, T. A. Love, J. W. MeConne11, J. F. Emery, K. J. Northcutt, and R. W. Peelle, "Delayed Beta- and Gama-Ray Production Due to Thermal-Neutron Fission of ${ }^{239} \mathrm{Pu}$ : Tabular and Graphical Spectral Distributions for Times After Fission Between 2 and 14000 sec," Oak Ridge Nationa1 Laboratory report NUREG/CR-1172, ORNL/NUREG-66 (January 1980).

3. J. L. Yarnell and P. J. Bendt, "Decay Heat from Products of ${ }^{235} \mathrm{U}$ Thermal Fission by Fast-Response Boil-Off Calorimetry," Los Alanos Scientific Iaboratory report LA-NUREG-6713 (September 1977). 
4. J. L. Yarnell and P. J. Bendt, "Calorinetric Fission Decay Heat Heasurements for ${ }^{239} \mathrm{Pu},{ }^{233} \mathrm{U}$, and ${ }^{235} \mathrm{U}$," Los Alamos Scientific Laboratory report NUREG/CR-0349, LA-7542-MS (September 1978).

5. E. T. Jurney, P. J. Bendt, and T. R. England, "Fission Product Gama Spectra," Los Alamos Scientific Laboratory report LA-7620-MS (January 1979).

6. R. J. LaBauve, T. R. England, and D. C. George, "Integral Data Testing of ENDF/B Fissi in Product Data and Comparisons of ENDF/B with Other Fission Product Dat wiles," Los Alamos National Laboratory report LA-9090-MS (December 1981).

7. Fission-Product Decay Library of the Evaluated Nuclear Data File, Version $V(E N D F / B-V)$. [Available from and maintained by the National Nuclear Data Center (NNDC) at Brookhaven National Laboratory]. NOTE: Spectral files in these compilations are based on data evaluated at INEL.

8. T. R. England, R. Wilczynski, and N. L. Whittemore, "CINDER-7: An Interim Report for Users," Los Alamos Scientific Laboratory report LA-5885-MS (April 1976). [CINDER-10, the version used in this report is unpublished. It is described in "Applied Nuclear Data Research and Development, January 1 - March 31, 1976," Los Alamos Scientific Laboratory report LA-6472-PR, p. 60 (1976), and in "Applied Nuclear Data Research and Development, October 1 - December 31, 1975," Los Alamos Scientific Laboratory report LA6266-PR, p. 13 (1976).]

9. "American National Standard for Decay Heat Power in Light Water Reactors," prepared by the American Nuclear Society Standards Comittee Working Group ANS-5.1, ANSI/ANS-5.1 (1979).

10. R. J. LaBauve, T. R. England, D. C. George, and M. G. Stamatelatos, "The Application of a Library of Processed ENDF/B-IV Fission-Product Aggregate Decay Data in the Calculation of Decay-Energy Spectra," Los Alamos Scientific Laboratory report LA-7483-MS (Sentember 1978).

11. R. J. LaBauve, D. C. George, and T. R. England, "FITPULS, A Code for Obtaining Analytic Fits to Aggregate Fission-Product Decay-Energy Spectra," Los Alamos Scientific Laboratory report LA-8277-MS (March 1980).

12. R. J. LaBauve, T. R. England, D. C. George, and C. W. Maynard, "Fission Product Analytic Impulse Source Functions," Nuc1. Tec. 56, 322 (February 1982). 


\section{APPENDIX A}

A full listing of the conputer code ADWM is contained in this appendix. The adjusted fits can be abstracted from the block data routine and used in other applications. The fits are in the order ${ }^{235} \mathrm{U}$ betas, ${ }^{235} \mathrm{U}$ ganas, ${ }^{230} \mathrm{Pu}$ betas, and ${ }^{239} \mathrm{Pu}$ garmas. For the beta fit, groups 1 and 2 have been combined; thus, in order to retrieve the fits for group 8 betas, one must abstract the seventh set of parameters. For nonspectral calculations of the total (beta plus gama) decay energy, the fits to the standard have been included; see subroutine STNDRD.

Input and output listings of a sample problem have been included following the listing of the code. This problen calculates the decay-energy spectra in 12 groups (see Table II), at 2 cooling tiwes $\left(10^{6}\right.$ and $10^{8} \mathrm{~s}$ ), from a reactor whose operating time was $7.2 \times 10^{7} \mathrm{~s}$, with a thermal flux of $10^{14} \mathrm{~m} / \mathrm{cm}^{2} / \mathrm{s}$, an epithermal flux of $5 \times 10^{14} \mathrm{n} / \mathrm{cm}^{2} / \mathrm{s}$, and with a fuel mixture $75 \%$ $23 \mathrm{E}_{\mathrm{U}}$ and $25 \%$ ${ }^{239} \mathbf{P u}$. 
Los Alamos Identification No. LP-1434

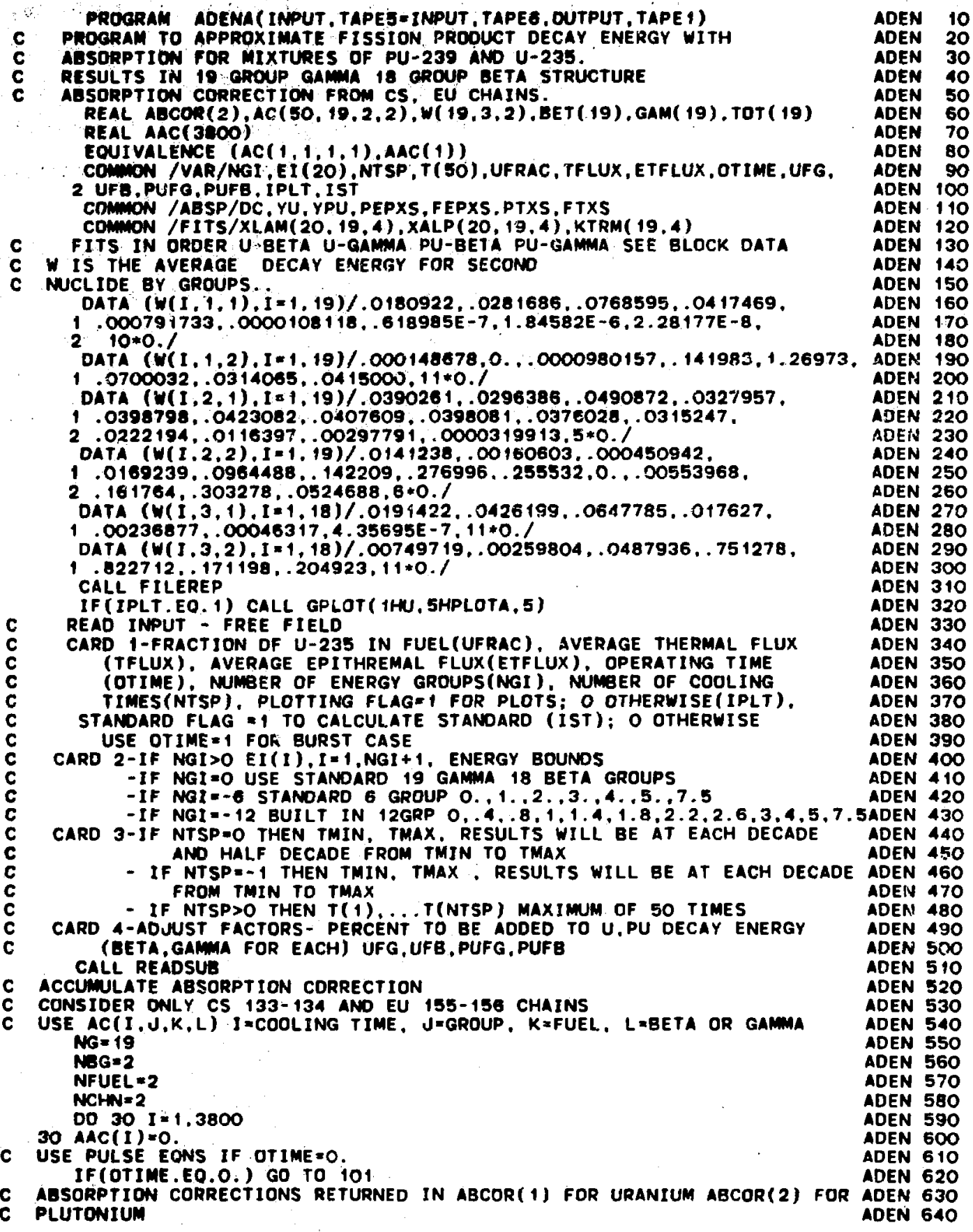




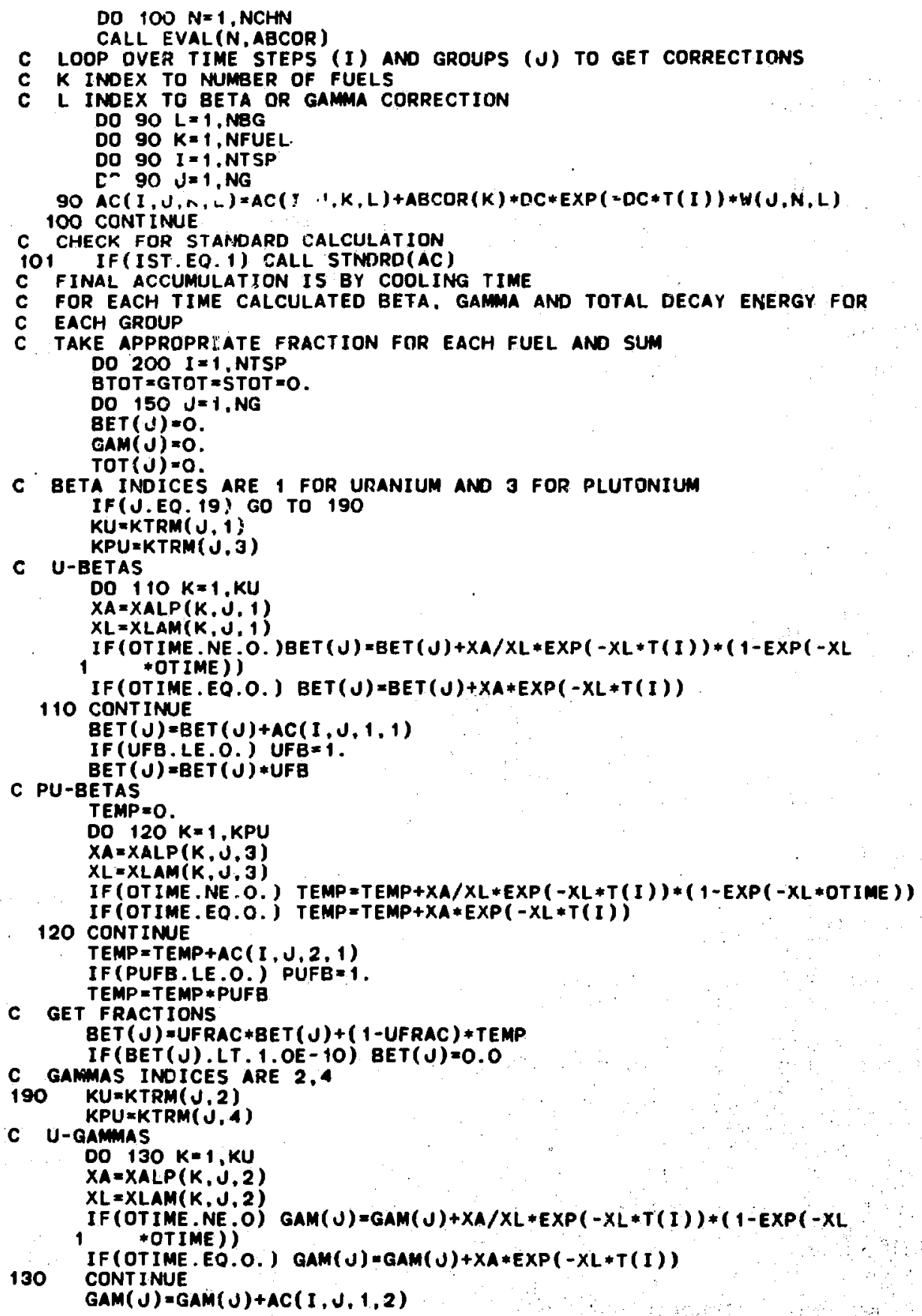

ADEN 650

ADEN 660

ADEN 670

ADEN 680

ADEN 690

ADEN 700

ADEN 710

ADEN 720

ADEN 730

ADEN 740

ADEN 750

ADEN 760

ADEN 770

ADEN 780

ADEN 790

ADEN 800

ADEN 810

ADEN 820

ADEN 830

ADEN B40

ADEN 850

ADEN 860

ADEN B70

ADEN 880

ADEN 890

ADEN 900

ADEN 910

ADEN 920

ADEN 930

ADEN 540

ADEN 950

ADEN 960

ADEN 970

ADEN 980

ADEN 990

ADEN1000

ADEN1O10

ADEN1020

ADEN 1030

ADEN1O4O

ADEN1OSO

ADEN1060

ADEN1O70

ADEN1OSO

ADEN109O

ADEN1 100

ADEN1110

ADEN1 120

ADEN1 130

ADEN1 140

ADEN1 150

ADEN1160

ADEN1 170

ADENI 180

ADEN1 190

ADEN 1200

ADEN1210

ADEN1220

ADEN1230

ADEN 1240

ADEN1250

ADEN1260

ADEN1270

ADEN 1280 
IF (UFG. LE. O.) UEO=1.

ADEN1290 $\operatorname{CAM}(U)=\operatorname{GAM}(U) * U F G$

C PU-GAminas

$\begin{aligned} & \text { TEMP }=0 \\ & \text { DO } 140 \\ & X\end{aligned}=1, K P U$

$X A=X A L P\{(K, j, 4)$

$X L=X L A M(K, J, 4)$

IF (OTIME. NE . O. ) TEMP = TEMP +XA/XL*EXP $(-X L * T(I)) *(1-E X P(-X L * O T$ IME ))

IF (OTIME.EO.O.) TEMP $=T E M P+X A * E X P(-X L * T(I))$

140. CONT INUE

TEMP = TEMP+AC $(1, J, 2,2)$

IF (PUFG. LE.O.) PUFG $=1$.

TEMP $=$ TEMP $* P U F G$

GAM(J) =UFRAC * GAM(J) + ( 1 -UFRAC ) * TEMP

IF(GAM(J).LT.1.E-10) GAM(J) $=0.0$

C Accuimulate TOTALS

$\operatorname{TOT}(J)=\operatorname{GAM}(J)$

IF $(J$.NE. 1) TOT $(J)=\operatorname{TOT}(J)+\operatorname{BET}(J-1)$

STOT =BTOT +BET $(J)$

GTOT = GTOT + GAM $(J)$

STOT = STOT+TOT (J)

150 CONT INUE

C REGRDUP IF NECESSARY

CALL REGROUP (BET, GAM, TOT)

C OUTPUT SECTION

WRI TE $(6,151)$ 151 FORMAT (1H1.T2O. "FISSION PRODUCT DECAY ENERGY FOR A MIXTURE OF U-23A

TEMP $=(1$. UFRAC $) * 100$.

TEMP $1=U F R A C * 100$.

WRITE (G, 152) TEMP 1, TEMP, TFLUX, ETFLUX, OTIME, T(I)

152 FORMAT (T3C. "PERCENT U-235", T50, IPE 11.4/

1 T30, "PERCENT PU-239", T50, IPE 11.4/

2 T3O. "THERMAL FLUX", T5O, IPE 11.4 " N/CM*2-5"/

3 T30; "EPITHERMLL FLUX", T5O, 1PE 1i.4," N/CM**2-5"/

4 T30. "OPERATING TIME", T50, TPE 11.4." SECONDS"/

5 T30, "COOLING, TIME", TSO, IPE 11.4," SECONOS")

WRITE $(6,160)$

160 FORMAT ( 1 HO, T 10.

2 " GRP ELO EHI BETA DEGAY ENERGY GAMMA UECAY ENERGY

1 TOTAL DECAY ENERGY")

WRITE $(6,161)$

161 FORMAT(T 10,7X, "(MEV) (MEV)".7X." (MEV/FIS)", 12X." (MEV/FIS)". 12 1X. "(MEV/FIS)")

WRITE (6,162) (J,EI (J),EI $(J+1), \operatorname{BET}(J), \operatorname{GAM}(J), \operatorname{TOT}(J), J=1, N G I)$

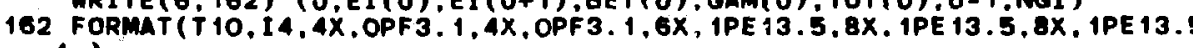
1 )

WRITE (6, 164) BTOT,GTOT, STOT

164 FORMAT(/T10," TOTALS OVER GROUPS ", 3X, 1PE 13.5, BX, IPE 13, 5, 8X, 1 1PE 13.5)

IF (IPLT.EO.O) CO TO 200

CALL PLOTIT(TOT, 1, I)

CALl Plotit (BET,2, I)

call plotit (Gam, 3, i j

200 CONT INUE

IF(IPLT.EO.O) GO TO 250

CALL DONEPL

CALL CDONE

250

STOP

END

SUBROUT INE REGROUP (BET. GAM, TOT)

COMMON /VAR/NGI, EI (20), NTSP. T (5O), UFRAC, TFLUX, ETFLUX, OT IME , UFG,

2 UFE, PUFG, PUFB, IPLT, IST

REAL E(20), BET(2), GAM(2), TOT(2),E1(7),E2(13)

ADEN1300

ADEN13 10

ADEN1320

ADEN 1330

ADEN1340

ADEN1350

ADEN1360

ADEN 1370

ADEN1380

ADEN 1390

ADEN1400

ADEN 1410

ADEN1420

ADEN1430

ADEN1440

ADEN 1450

ADEN1460

ADEN1470

ADEN1480

ADEN1490

ADEN1500

ADEN15 10

ADEN 1520

ADEN 1530

ADEN 1540

ADEN 1550

ADEN 1560

ADEN 1570

ADEN 1580

ADEN 1590

ADEN 1600

ADEN\&Õ to

ADEN 1620

ADEN1630

ADEN1640

ADEN 1650

ADEN1660

ADEN 1670

ADEN 1680

ADEN 1690

ADEN1700

ADEN1710

ADEN1720

ADEN1730

SADEN 1740

ADEN1750

ADEN1760

ADEN 1770

ADEN1780

ADEN1790

ADEN 1800

ADEN18 10

ADEN1820

ADEN 1830

ADEN 1840

ADEN 1850

ADEN 1860

ADEN1870

ADENTE8O

REGR 10

REGR 20

REGR 30

REGR 40 
DATA E/O.,.1,.2,.4,6, 6,1,.1.2,1.4,1.6,1.8,2.,2.2.2.4.

REGR 50 $12.6 .3 .4 .5 .6 .7 .5 /$

DATA E1/0.,1,2, 3, ,4,5.,7.5/

DATA E2/0..4.8,1.1.4, $1.8,2.2,2.6,3.4,5.6 .7 .5 /$

SHIFT BETA GROUPS DOWN SO GROUP 1 IS EMPTY

DO $2 . I=1,18$

$2 \operatorname{BET}(19-I+1)=\operatorname{BET}(19-I)$

$\operatorname{BET}(1)=0$.

C STANDARD 19 GROUPS

IF (NGI. NE. 19) GO TO 5

DO $3 I=1,20$

$3 E I(I)=E(I)$ RETURN

C. LOOK FOR STANDARD 6 AND 12 GROUPS

5 IF(NGI.GT.O) GO TO 9

IF (NGI.NE. -6) GO TO 7

NGI $=6$

Do $6 I=1,7$

$6 \quad E I(I)=E I(I)$

$7 \quad$ GO 709

DO $8 I=1,13$

$B \quad E I(I)=E 2(I)$

$5 \quad N G=20$

USTART $=1$

DO $100 \mathrm{I}=1$, NGI

DO $10 \mathrm{~J}=\mathrm{JSTART}$, NG

C FIND HOW MANY GROUPS TO COMBINE

IF(EI $(I+1) . E Q . E(J))$ GO TO 20

10 CONT INUE

WRITE $(6,15)$

15 FORMAT (" ILLEGAL ENERGY BOUNDS SPECIFIED") STOP

$20 \quad T 1=0$.

$\mathrm{T} 2=0$.

$T 3=0$.

JEND $=J-1$

DO $30 K=$ JSTART , JEND

$T 1=T 1+B E T(K)$

$T 2=T 2+\operatorname{GAM}(K)$

$30 \mathrm{~T} 3=\mathrm{T} 3+\mathrm{TOT}(K)$

$B E T(I)=T 1$

$\operatorname{GAM}(I)=T 2$

$\operatorname{TOT}(I)=13$

USTART $=$ J

100 CONTINUE

RE TURN

END

SUBROUTINE READSUB

COMMON /VAR/NGI, EI (20), NTSP, T(50), UFRAC, TFLUX, ETFLUX, OTIME, UFG,

2 UFB, PUFG, PUFB, IPLT, IST

READ *, UFRAC, TFLUX, ETFLUX, OTIME, NGI , NTSP, IPLT IST

NG1 $=$ NGI + 1

IF (NG1.LT.21) GO TO 10

WRITE $(6,8)$

8 FORMAT (" MAX OF 20 GROUPS") STOP

10 IF (NGI.GT.O) READ *., (EI (I), I =1.NG1)

IF (NGI.EQ.O) NGI $=19$

IF (NTSP.GT.O) GO TO 30

READ * TMIN, TMAX

IF (TMIN.NE. TMAX) GO TO 15 NT SP $=1$

$T(1)=T M I N$

REGR 60

REGR 70

REGR 80

REGR 90

REGR 100

REGR 110

REGR 120

REGR 130

REGR 140

REGR 150

REGR 160

REGR 170

REGR 180

REGR 190

REGR 200

REGR 210

REGR 220

REGK 230

REGR 240

REGR 250

REGR 260

REGR 270

REGR 280

REGR 290

REGR 300

REGR 310

REGR 320

REGR 330

REGR 340

REGR 350

REGR 360

REGR 370

REGR 3EC

REGR 390

REGR 100

RE GR 410

RECR 420

REGR 130

REGR 440

REGR 450

REGR 460

REGR 470

REGR 480

REGR 490

REGR 5CO

REGR 510

REGR 520

READ 10

READ 20

READ 30

READ 40

READ 50

READ 60

READ 70

READ 80

READ 90

READ 100

READ 110

READ 120

READ 130

REEAD 140

REAÓ 150

REAO 160 
60 To 40

READ 170

$15 L=I F I X(A L O G 10(T M I N))$

IF ( $L . L T . O) L=L-1$

$M=$ IFIX (ALOG IO (TMAX)+.99)

IF (NTSP, EO, - 1) GO TO 20

NT $S P=2 *(M-L)+1$

$J=1$

DO $18 I=1$.NTSP, 2

$T(I)=10 . *(L+J-1)$

$T(I+1)=5 * T(I)$

$J=v+1$

18 CONT INUE

$C=$ TO 40

20 NTSP $=(M \cdot L)+1$

DO $22 I=1$. NTSP

$22 T(I)=10 . *(L+1-1)$ Go To 40

30 IF(NTSP.LE.50) GO TO 35

WRITE $(6,32)$

32

FORMAT(" MAX OF 50 TIME STEPS") STOP

35 READ * (T (I), I = + .NTSP )

40 READ *UFG,UFG, PUFG, PUFB

RETURN

END

SUBROUT INE EVAL (NCID, ABCOR)

COMMON /VAR/NGI, EI (20), NTSP, T( 50), UFRAC, TFLUX, ETFLUX, OTIME, UFG.

2 UFB, PUFG, PUFE, IPLT, IST

COMNON /AESP/DC, YU, YPU, PEPXS, FEPXS, PTXS, FTXS

REAL ABCOR (2), K

C MCID - NUCLIDE ID 1 FOR CS 2 FOR EU

C DC DECAY CONSTANT.

C YU CUMULATIVE YIELD FRACTION FROM THERIAL FISSION U-235

C YPU CUMULATIVE YIELD FRACTION FROM THERMAL FISSION PU-239

PEPXS (N.GAM) XS OF PRECURSOR EPI THERMAL

FEPXS(N,GAM) XS OF SECOND NUCLIDE

PTXS(N, GAM) XS OF PRECURSOR THERMAL

FTXS (N,GAM) XS OF SECOND NUCLIDE

K CONSTANT

EVALUATE ADDITIONAL ATOM DENSITY OF NUCLIDE 2 RESULTING

FROM RADIATIVE CAPTURE IN NUCLIDE 1.

THIS TERM IS INOEPENDENT OF GROUP OR COOLING TIME.

ABCOR( 1) FOR $U$ AECOR( 2 ) FOR PU

$Y * A * / K(1 /(A * B)-(\operatorname{EXP}(-A * O T I M E) /(A *(B-A)))+(\operatorname{EXP}(-B * O T I M E) /(B *(B-A))))$

A-PTXS*TFLUX+PEPXS*ETFLUX

$B=D C+F T X S * T F L U X+F E P X S * E T F L U X$

K.1.0

TÉFINID.EO.1) CALL CSCHN

IF (NCIO.EQ.2) CALL EUCHN

$A=(P T X S * T F L U X+P E P X 5 * E T F L U X) * 1, E-24$

$B=D C+(F T X S * T F L U X+F E P X S * E T F L U X) * 1 . E-24$

$A B C O R(1)=A * K *(1 /(A * B)-(\operatorname{EXP}(-A *$ OT $[M E) /(A *(B-A)))+$

1 (EXP $(-B * 0 T 1 M E) /(B *(B-A))))$

ABCOR $(2)=A B C O R(1) * Y P U$

$\operatorname{ABCOR}(1)-A B C O R(1) * Y U$

RETURN

END

SUBROUTINE CSCHN

COMinion /ABSP/X( 7$)$

REAL AR ( 7)

DATA AR/ 1.06523E-8, .06779,0.06957,34, 12,20.454,30,162,140.67/

C FILL ABSP COMMON WITH VALUES FOR CS CHAIN

Do $10 I=1,7$

$10 X(I)=A R(I)$

READ 180

REAO 190

READ 200

READ 210

READ 220

READ 230

READ 240

READ 250

READ 260

READ 270

READ 280

READ 290

READ 300

READ 310

READ 320

READ 330

READ 340

READ 350

READ 360

READ 370

READ 380

READ 390

REAO 400

READ 410

EVAL 10

EVAL 20

EVAL 30

EVAL 40

EVAL 50

EVAL 60

EVAL 70

EVAL 80

EVAL 90

EVAL 100

EVAL 110

EVAL 120

EVAL 130

EVAL 140

EVAL 150

EVAL 160

EVAL 170

EVAL 180

EVAL 190

EVAL 200

EVAL 210

EVAL 220

EVAL 230

EVAL 240

EVAL 250

EVAL 260

EVAL 270

EVAL 280

EVAL 290

EVAL 300

EVAL 310

EVAL 320

CSCH 10

CSCH 20

CSCH 30

CSCH 40

CSCH 50

CSCH 60

CSCH 70 


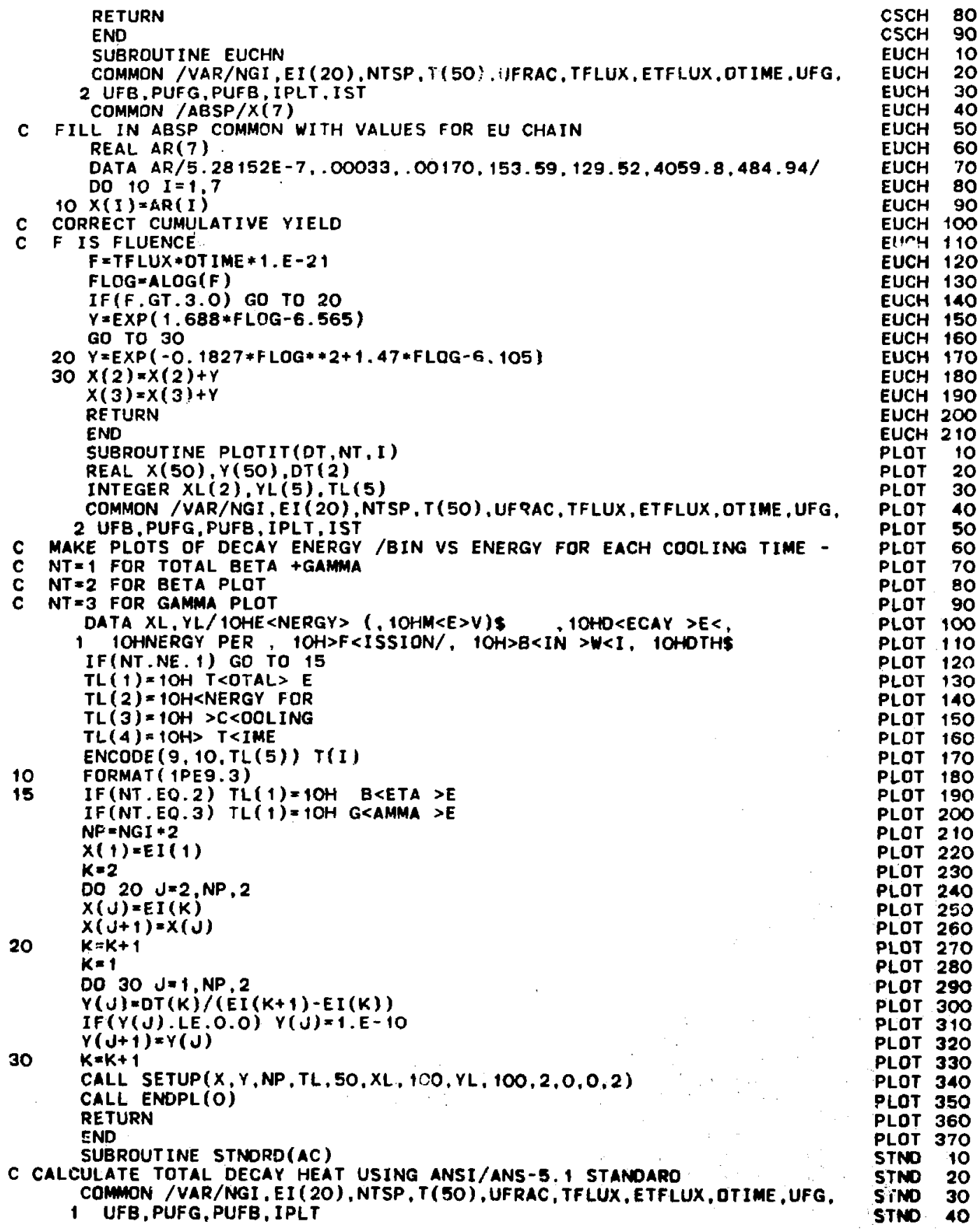


REAL STL $(23,2), \operatorname{STA}(23,2), \operatorname{ST}(50), A C(50,19,2,2) \quad$ STND 50

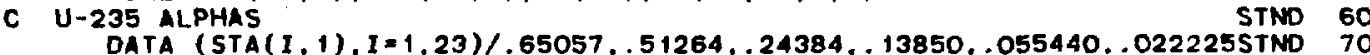

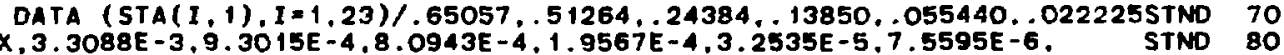

$\mathrm{X} 2.5232 \mathrm{E}-6.4 .9948 E-7.1 .8531 E-7.2 .6608 E-8.2 .2398 E-9.8 .1641 E-12 . \quad$ STND 90

$\times 8.7797 E-11.2 .5131 E-14.3 .2176 E-16.4 .5038 E-17.7 .4791 E-17 / \quad$ STND 100

C U-235 LAMBDAS

STND 410

DATA (STL $(I, 1), 1=1,23) / 22.138, .51587, .19594, .10314, .033656, .011681$ STND 120

$x \quad 0035870,0013930,6.2630 E-4,1.8906 E-4.5 .4988 E-5.2 .0958 E-5, \quad$ STND 130

$\times \quad i .001 E-5,2.5438 E-6,6.6361 E-7,1.2290 E-7.2 .7213 E-8,4.3714 E-9, \quad$ STND 140

$\times 7.5780 E-10,2.4786 E-10$.

$\times 2.2384 E-13,2,46 E-14,1.5699 E-14 /$

C PU-239 ALPHAS

2) $I=1.23)(2093.3853 . .2213,09460.03531 .002292$.

$\times .003946, .001317,7.052 E-4,1.432 E-4,1.765 E-5,7.347 E-6, \quad$ STND 190

$\times 1.747 E-6,5.481 E-7,1.671 E-7.2 .112 E-8,2.996 E-9,5.107 E-11.5 .730 E-11$. STNO 200

X 4.138E-14,1.088E-15.2.454E-17.7.557E-17/
STAD 210

C PU-239 LAMBDAS

DATA $(\operatorname{STL}(I, 2), I=1.23) / 10.02, .6433 \ldots 2186, .1004 \ldots 03728 \ldots 01435$.

$\times .004549, .001328,5,356 E-4,1.73 E-4,4.881 E-5,2.006 E-5$,

$X 8.319 E-6,2.358 E-6,6.450 E-7,1.278 E-7,2.466 E-8,9.378 E-9$.

$\times 7.45 E-10.2 .426 E-10.2 .21 E-13.2 .64 E-14,1.38 E-14 /$

C STANDARD FOR TOTALS ONLY

DO $100 I=1$. NTSP

$\mathrm{ST}(\mathrm{I})=0$.

DO $90 \mathrm{~J}=1.2$

$5=0$.

C LOOP THRU URANIUM THEN PLUTONIUM CALC

C SEE IF BURST OR FINITE IRRADIATION CALC IF (OTIME. NE.O.) GO TO 20 DO $10 K=1,23$

$10 S=S+S T A(K, J) * E X P(-S T L(K, J) * T(I))$ GO TO BO

$200022 K=1.23$

STND 220

STND 230

STND 240

STND 250

STND 260

STND 270

STND 280

STND 290

STND 300

STND 310

STND 320

STND 330

STND 340

STND 350

STND 360

STND 370

STND 380

$22 S=5+S T A(K, J) / S T L(K, J) * \operatorname{EXP}(-S T L(K, J) * T(1)) *(1-\operatorname{EXP}(-S T L(K, J) * 0 T 1 M E)) S T N D 390$

C ADO IN ALL ABSORPTION CORRECTIONS

DO $30 K=1,2$

DO $30 L=1,19$

$30 \mathrm{~S}=S+A C(1, L, U, K)$

C FIGURE-PER CENT FOR U AND PU

80 IF (U.EQ.1) ST(I) =UFRAC*S

IF (U.EQ.2) ST (I) $=5 T(I)+(1-$ UFRAC $) * S$

go CONTINUE

100 CONTINUE

C PRINT RESULTS

TEMP $1=$ UFRAC * 100

$T E M P=100$. - TEMP 1

WRITE $(6$, i5o)

150 FORMAT ( 1 H1, T20, "CALCULATION OF ANSI/ANS-5. 1 STANDARD")

WRITE $(6,152)$ TEMP 1, TEMP, TFLUX, ETFLUX, OT IME

152 FORMAT (T30, "PERCENT U-235". T5O. IPE $11.4 /$

1 T30. "PERCENT PU-239", T5O, 1PE 11.4/

2 T30. "THERMAL FLUX". T5O IPE 11.4" N/CM" $2-5 " /$

3 T30. "EPI THERMAL FLUX", T50, IPE 11.4." N/CM" "2-S"/

4 T30, "OPERATING TIME", T5O, TPE 1 1.4." SECONOS")

WRITE $(6,154)$

154 FoRMAT ( //T 10. "COOLING TIME". T4O. "TOTAL DECAY ENERGY" /

1 T 15, "(SEC)". T45, "(MEV/FIS)" )

WRITE (6,156) (T(i),ST (I), I=1,NTSP)

156 FORMAT (T12, IPE 12.5, T42, IPE 12.5)

STOP

RETURN

END

BLOCK DATA

STND 400

SIND 410

STND 420

STND 430

STND 440

STND 450

STND 460

STND 470

STND 480

STND 490

STND 500

STND 510

STND 520

STND 530

STND 540

STND 550

STND 560

STND $\mathbf{5 7 0}$

STND 580

STND $\mathbf{5 9 0}$

STND 600

STND 610

STND 620

STND 630

STND 640

STND 650

STNO 660

STND 670

Data 10 
COMMON /FITS/ XLAM(20,19,4),XALP(20,19,4),KTRM(19,4) DATA 20

C FITS FOR U-235 BETAS

DATA 25

DATA (XALP(K, 1,1),K=1,20)/

DATA 30

$X .6434 E-11, .1913 E-10, .1578 E-09, .2530 E-08, .1708 E-07, .4206 E-07.0 A T A$ 40

$X .1834 E-06, .5005 E-06, .2179 E-05, .8087 E-05, .2306 E-04 ; .9554 E-04.0 A T A$ 5O

$x .5491 E-03 ; 0.1982 E-02 ; .1696 E-02,-.6758 E-03, .6054 E-03,-.2326 E-03$. DATA 6O
$X 0$.

Xo.

DATA 80

DATA (XLAM(K, 1, 1),K=1,20)/

$x .1780 E-04 ; .7042 E-04, .1454 E-03 ; .4018 E-03, .1345 E-02, .7228 E-02$, 0ATA 100

$X .3395 E-01, .1833 E+\infty 0, .6551 E+00, .8562 E+\infty 0, .8475 E+01 . .4550 E+01$. DATA 110

Xo. $0 . \quad$ DATA 120

DATA (XALP $(K, 2,1), K=1,20) / \quad$ DATA 130

$X .1062 E-10, .1890 E-11, .6351 E-10, .2015 E-08, .1576 E-07, .5459 E-07.0 A T A$ 140

$X .6061 E-06, .9710 E-06, .3984 E-05, .1727 E-04, .5401 E-04, .2774 E-03.0 A T A$ 15O

$X .8341 E-03, .9005 E-03, .5944 E-02,-.2499 E-01, .2153 E-01, .1110 E-01$. DATA 160

$X-.1126 E-01,0$.

DATA (XLAM(K, 2,1$), K=1,20) /$

DATA 170

X 7807E-G9 $1148 E-07,3184 E-07$ 1585E-06, 6529E-06, 3463E-05 DATA 180

$x .1731 E-04, .6245 E-04, .1701 E-03 ; .4936 E-03, .1393 E-02, .9581 E-02.0 A T A 200$

$X .3740 E-01, .1121 E+00, .4593 E+00, .7693 E+00, .8770 E+00, .1010 E+02.0 A T A 210$

$X .9788 E+01.0$.

DATA (XALP(K, 3,1$), K=1,20) /$

DATA 220

$X \quad 4750 E-11,0357 E-11,7888 E-10,2391 E-08,054 E-07,0220 E-07$ DATA 230

X.6783E-06, . 1340E-05, .9950E-05,.6032E-04, .3861E-03,. 1226E-02.0ATA 250

$X .2435 E-02, .2601 E-02, .5654 E-02, .5239 E-02,-.8991 E-02,-.1611 E-05$, DATA 260

Xo. $0 . \quad$ DATA 270

DATA (XLAM(K, 3,1),K=1,20)/

$X .7805 E-09, .1191 E-07, .3140 E-07, .1698 E-06, .7808 E-06, .4453 E-05$. DATA 290

$X .1624 E-04, .6349 E-04, .1951 E-03, .9275 E-03, .7555 E-02, .3455 E-01$. DATA 300

$X .1381 E+00, .3703 E+00, .1792 E+01, .5809 E+01, .3458 E+01, .1178 E-03$. DATA 310

Xo. 0.0 DATA 32O

DATA (XALPIK, 4,1),K=1.20)/ DATA 330

$X .4883 E-11, .1903 E-10, .9872 E-10, .4300 E-08, .1432 E-07, .1350 E-06$, DATA 340

$X .9369 E-06, .5395 E-05, .7592 E-04, .1308 E-02, .267$ 1E-02,.93 15E-02.DATA 350

$X .7551 E-03,-.7696 E-02 ; .9081 E-02,-.5847 E-03,-.7231 E-04, .8844 E-04, D A T A$ 360

$X-1824 E-08,0$.

DATA (XLAM(K, 4,1),K=1,20)/ DATA 380

$X .7499 E-09, .2138 E-07, .3127 E-07, .1626 E-06, .7559 E-06, .5469 E-05$, OATA 390

$X .2151 E-04, .1349 E-03, .5565 E-03, .9635 E-02, .4599 E-01, .3322 E+00, D A T A 400$

$\mathrm{X} .1206 \mathrm{E}+02, .8495 \mathrm{E}+00, .1442 \mathrm{E}+01, .9493 \mathrm{E}-02, .6678 \mathrm{0}-03, .1281 \mathrm{E}-02$, DATA 410

$X .1742 E-06,0$.

DATA (XALP(K, 5,1$), K=1,20) \%$

DATA 420

.6292E-11,.1042E-09,.6666E-10,.2504E-08,.1152E-07, .6957E-06. DATA 440

.644E-04,.635E-04,.1760E-01,.2071E-01,-.2346E-01, -.1872E-01.DATA 450

$\mathrm{X} .3231 \mathrm{E}-02, .6294 \mathrm{E}-02, .1352 \mathrm{E}-01,-.5361 \mathrm{E}-06, .1231 \mathrm{E}-05,0 . \quad$, DATA 460

Xo. O.

DATA (XLAMIK, 3,1$), K=1,20)$ /

DATA 470

$X .7685 E-09, .2614 E-07, .3809 E-07, .1591 E-06, .1020 E-05, .8643 E-05.0 A T A$ 490

$X .1924 E-03 ; .9142 E-03 ; .1071 E-01 ; .4203 E+01, .3437 E+01, .1116 E-01$, DATA SOO

$\mathrm{X} .1991 \mathrm{E}-01, .1267 \mathrm{E}+00, .1096 \mathrm{E}+01, .9227 \mathrm{E}-05, .3095 \mathrm{E}-04,0$. ,DATA 510

Xo. 0 .

DATA (XALP(K, 6,1$), K=1,20) /$

DATA 520

$X .7112 E-11 . .1540 E-09, .6938 E-10, .2156 E-08, .5464 E-08, .6190 E-07.0 A T A$ 530

$X .4927 E-06, .2253 E-05, .1857 E-04, .6168 E-04, .2965 E-03, .1574 E-02$. DATA S5O

$X .357 R E+00,-.3686 E+00 ; .2491 E-01, .7847 E-02,-.2409 E-02,-.4072 E-09$. DATA S6O

Xo. 0 .

DATA (XLAM(K, 6,1),K=1,20)/ $\quad$ DATA 580

$X .7706 E-09, .2641 E-07, .4577 E-07, .1623 E-06, .9591 E-06, .7049 E-05.0 A T A$ S90

$X .2044 E-04, .6548 E-04, .3281 E-03, .1229 E-02, .6261 E-02, .1967 E-01$, DATA .600

$x .1663 E+00 ; .1708 E+00 ; .2903 E+00 ; .3439 E+01 ; .1017 E+02 ; .1777 E-06.0 A T A$ G10

DÁTA (XALP $(K, 7,1), K=1,20) /$

DATA 630 
$X .1339 E-05, .2520 E-05, .1466 E-04, .4227 E-04, .1311 E-03, .8081 E-03.0 A T A 650$ $x .1912 E-02, .5576 E-02 ; .3570 E-02, .2204 E-01,-.1461 E-01, .8132 E-02$. DATA 660
$X-.4068 E-10,0$.

DATA (XLAM(K, 7.1),K=1.20)

DATA 670

$X .7709 E-09, .2619 E-07, .3811 E-07, .1811 E-06, .1336 E-05, .1290 E-04$, DATA 690

$X .3986 E-04, .1399 E-03, .3666 E-03, .1039 E-02, .4132 E-02 ; .1239 E-01$. DATA 700

$X .3473 E-01, .1258 E+00 ; .1675 E+00, .9831 E+00, .1783 E+01, .4127 E+01$. DATA 710

$\times$.2837E-05.0.

DATA (XALP(K, 8,1$), K=1,20) /$

DATA 720

$X .6463 E-11, .1825 E-09, .8885 E-10, .3097 E-09, .8137 E-09, .2905 E-08$, DATA 740

$X .1905 E-06, .1107 E-05, .1110 E-05, .1731 E-04, .4208 E-04, .9869 E-04$.DATA 750

$\mathrm{X} .8968 \mathrm{E}-03, .2568 \mathrm{E}-02, .5091 \mathrm{E}-02, .1612 \mathrm{E}-01, .3812 \mathrm{E}-01,-.3097 \mathrm{O}-01$, DATA 760

Xo.

$(k, 8,1), k=1,20) /$

DATA 770

DATA (XLAM(K, 8,1$), K=1,20) / \quad$ DATA 780

$X .7583 E-09, .2675 E-07 . .3901 E-07, .3983 E-06, .9840 E-06, .3216 E-05$, DATA 790

$X .1406 E-04, .4059 E-04, .8726 E-04, .3789 E-03, .1079 E-02, .3565 E-02$, DATA 800

$x .1219 E-01, .4522 E-01 ; .1086 E+00, .3837 E+00, .3853 E+01, .4879 E+01$. DATA B 10

Xo 0 .

DATA 820

DATA (XALP(K,, 1$), K=1,20) /$

DATA 830

$X .4849 E-11, .2136 E-09, .6448 E-10, .1028 E-08, .6465 E-07, .1052 E-05 . D A T A$ B4O

$X .2872 E-05, .2114 E-02, .1843 E-01,-.7584 E-02, .2210 E-01,-.2356 E-02,0 A T A$ B5O

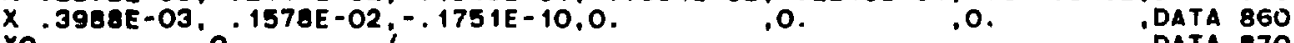

DATA B70

DATA (XLAM(K, 9,1$), K=1,20) /$

DATA 880

$\times .7314 E-09, .2655 E-07.5161 E-07, .1138 E-05, .1148 E-04 . .3500 E-04$, DATA B9O

$X .1536 E-03, .1078 E-02, .1263 E+00, .1502 E+00, .7820 E+00, .1131 E-02$, DATA 900

$X .1823 E-02, .1764 E-01 ; .4980 E-07.0 .0 .0 .0 .040$

XO.

DATA (XALP(K, 10,1), K=1,20)/ DATA 930

$X .2949 E-11, .1980 E-09, .6559 E-10, .5338 E-09, .8019 E-07 . .1207 E-05 . D A T A$ 940

$\mathrm{X} .2281 \mathrm{E}-04, .5828 \mathrm{E}-04, .4859 \mathrm{E}-03, .9572 \mathrm{E}-02, .1438 \mathrm{E}-01, .2216 \mathrm{E}-01$. DATA 950

$X-.6902 E-02,-.9159 E-07,-.2522 E-10.0 . \quad .0 . \quad 0 . \quad$,DATA 960

Xo. $0 . \quad ; \quad$ DATA 970

DATA (XLAM(K, 10, 1), K=1,20)/

DATA 980

$X .7714 E-09, .2751 E-07, .3731 E-07, .1322 E-05, .1422 E-04, .4057 E-04.0 A T A 990$

$X .4868 E-03, .1670 E-02, .8908 E-02, .4377 E-01, .1733 E+00, .1193 E+01$, DATA 1000

$X .4579 E-01, .2355 E-02, .4412 E-07,0 . \quad .0 . \quad 0 . \quad$.0ATA1010

Xo. $0 . \quad$ DATA1020

DATA (XALP(K, 11,1),K=1,20)/ DATA1030

$X .1020 E-11, .1452 E-09, .7995 E-10, .2193 E-09, .2169 E-06, .1707 E-05.0 A T A 1040$

$X .2067 E-04$, .7755E-04;.6905E-03,.25/7E-02,. 1233E-01,.2177E-01, DATA 1050

$X .4179 E-02,-.1870 E-03,-.8830 E-06, .1946 E-10,0 . \quad .0 . \quad$ DATA 1060

Xo. $0 . \quad$ DATA1070

DATA (XLAM(K, 11,1),K=1,20)/ DATA1080

$X .7529 E-09, .2621 E-07, .3554 E-07, .2239 E-05, .2054 E-04, .5446 E-04$, DATA 1090 $X .5119 E-03, .2029 E-02, .1264 E-01 ; .4546 E-01, .1633 E+00 ; .7341 E+00,0 A T A 1100$ $\mathrm{X} .8797 E+01 ; .6310 \mathrm{E}-01 ; .6605 E-04, .3456 \mathrm{E}-07.0 . \quad .0$.
$\mathrm{X0.}$

DATA $(X A L P(K, 12,1), K=1,20) /$

DATA 1130

$X .1903 E-12, .1072 E-09 . .5483 E-10 . .1553 E-09, .1454 E-06 . .1201 E-05$. DATA 1140 $X .1695 E-04, .5651 E-04$. 3952E-03,.4120E-02,.7799E-02,.1116E-01.DATA1150

$X .2257 E-01,-.2236 E-02 ;-.1368 E-05,-.6888 E-11,0$.
$X 0$.

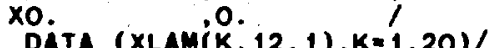

DATA1180

$X .7383 E-09, .2529 E-07, .3435 E-07, .2998 E-05, .2031 E-04, .5467 E-04$, DATA 190

$X .4881 E-03, .1594 E-02, .9282 E-02, .4844 E-01, .1677 E+00, .2477 E+00 . D A T A 1200$ $\mathrm{X} .1370 E+01 ; .8750 E-01 ; .1320 E-03, .2175 E-07.0$.
$\mathrm{XO} .0$.

xo.

DAtA (XALP $(K, 13,1), K=1,20) /$

DATA 1230

$X .1537 E-12, .8363 E-10, .2375 E-10, .4104 E-07, .6387 E-06, .2477 E-04$, DATA 1240

$X .1345 E-02, .7314 E-03, .4027 E-02, .3552 E-01,-.2066 E-01, .2439 E-01$. DATA 1250

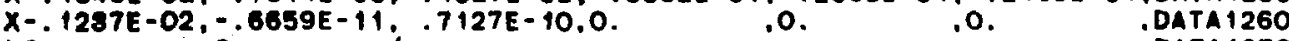

DATA $(X \operatorname{LAM}(K, 13,1), K m 1,20) /$

DATA1270

DATA 1280 
$X .7277 E-09, .2623 E-07, .4445 E-07, .1752 E-04, .4300 E-04, .6432 E-03, D A T A 1290$ $X .3340 E-02, .1420 E-01, .6944 E-01, .2643 E+00, .2914 E+00, .1245 E+01$, DATA 13O0 $X .3420 E-02, .4485 E-07 ; .2409 E-05.0$, , $0.0 . \quad$.DATA1310 XO. 0 . $\quad$ O DATA 1320 DATA (XALP $(K, 14,1), K=1,20) /$ DATA 1330 $X .2727 E-12, .4138 E-10, .1706 E-10, .1045 E-10, .1495 E-09, .1240 E-07$, DATA1340 $X .1340 E-05, .2917 E-04, .5145 E-04, .4355 E-03, .1236 E-01 . .4517 E-01$. DATA 1350 $X .457 O E-01,-.2425 E-01,-.2114 E-01, .2968 E-01,-.4287 E-06,0 . \quad$,DATA1360 XO. 0.

DATA $(X \operatorname{LAM}(K, 14,1), K=1,20) /$

DATA 1370

DATA 1380

$X .7243 E-09, .2441 E-07, .3570 E-07, .2328 E-06, .3553 E-05, .1636 E-04$, DATA 1390 $X .4797 E-04, .5905 E-03 . .1357 E-02, .6814 E-02, .4479 E-01, .6053 E+00 . D A T A 1400$ $X .3446 E+01, .4152 E+01 ; .6306 E-01, .1055 E+00, .8224 E-04,0 . \quad$,DATA 1410 Xo. 0.

DATA (XALP(K.15,1) $K=1,20)$ )

DATA 1420

DATA1430

$X .4153 E-12, .3579 E-11, .1346 E-11, .1425 E-10, .3418 E-09, .8996 E-06$, DATA1440

$X .2150 E-05, .4837 E-04, .9929 E-04, .3157 E-03, .2987 E-02, .3174 E-01$, DATA 1450 $X .1002 E+00, .4835 E-03,-.3577 E-01, .9453 E-01,-.1804 E-05,0 . \quad$,OATA1460 Xo. 0.

DATA 1470

DATA (XLAM(K, 15, 1),K=1,20)/

DATA 1480

$X .7098 E-09, .2083 E-07, .6657 E-07, .2455 E-06, .5361 E-05, .3918 E-04$, DATA 1490

$X .5184 E-04, .6238 E-03, .1948 E-02, .6540 E-02, .2109 E-01, .1171 E+00$, DATA 1500 $X .4571 E+\infty 0, .3940 E+01, .4790 E+00, .1663 E+01, .4392 E-04,0$. ,DATA1510 XO

DATA 1520 DATA 1530

DATA (XALP $(K, 16,1), K=1,20)$ /

$X .1360 E-12, .2872 E-11, .3918 E-09, .1941 E-06, .5200 E-05, .4277 E-04$,DATA 15AO $X .3132 E-03, .1951 E+00,-.4793 E+00, .3067 E+00, .7505 E-01, .4923 E-01$. DATA 1550 $X-.2039 E-01,-.8067 E-12, .5015 E-11,0 . \quad, 0 . \quad 0 . \quad$ DATA1560 Xo. $0 . \quad$ O DATA1570 DATA (XLAM $(K, 16,1), K=1,20) /$ DATA 1580

$X .7006 E-09, .2105 E-06 . .9443 E-05, .4775 E-04, .5230 E-03 . .3298 E-02$. DATA 1590 $X .9846 E-02, .7072 E-01, .7851 E-01, .8639 E-01, .6381 E+O 0 . .1712 E+01$, DATA 1600 $X .7458 E+00, .4439 E-06, .1375 E-05,0 . \quad .0 . \quad 0 . \quad$.DATA1610 Xo. 0 . ; $1375 E-05,0$

DATA 1620

DATA (XALP $(K ; 17,1), K=1,20) /$

DATA 1630

$X .3393 E-13, .5026 E-13, .1891 E-12, .1251 E-09, .7343 E-09, .2219 E-07$, DATA 16AO $X .1196 E-05, .2789 E-04, .1550 E-03, .1477 E-01, .2050 E-01,-.1423 E-01$, DATA 1650 $X .2480 E-01,-.1599 E-01,-.5153 E-08.0$, , $0 . \quad 0 . \quad$ DATA 1660 Xo. .0 .

DATA 1670

DATA (XLAM(K, 17,1$), K=1,20)$ )

DATA1680

$X .6959 E-09, .2317 E-06, .1891 E-05, .1121 E-04, .3562 E-04, .7610 E-04,04 T A 1690$ $X .4750 E-02, .4628 E-02, .2096 E-01, .1399 E+00, .6032 E+00, .5545 E+00$. DATA 1700 $X .2415 E+01, .3674 E+01 ; .7165 E-04.0$. . $0.0 . \quad$.DATA1710 XO. XALP (K. 18,1$), K=1,20) /$

$X 1.90934 E-15,5.36465 E-14,2.33: 81 E-11,2.77998 E-10,4.54551 E-8$ DATA 1720 DATA 1730

$\times \quad 1.93885 E-6,8.71837 E-5,2.22677 E-3,5.98149 E-3,8.85817 E-3$

$X 1.61727 E-3,-2.36961 E-10,-4.45492 E-14,7 * 0.1$

DATA $(X \operatorname{LAM}(K, 18,1), K=1,20) /$.

$X 7.06343 E-10,8.33849 E-7,1.16847 E-5,6.74779 E-5,1.36779 E-3$.

$X 4.87554 E-3,2.05905 E-2,7.64181 E-2,1.98977 E-1,1.04123$,

$\times 5.21095,7.60620 E-5,9.28661 E-7,7 * 0.1$ DATA (XALP $(K, 19,1), K=1,20) /$

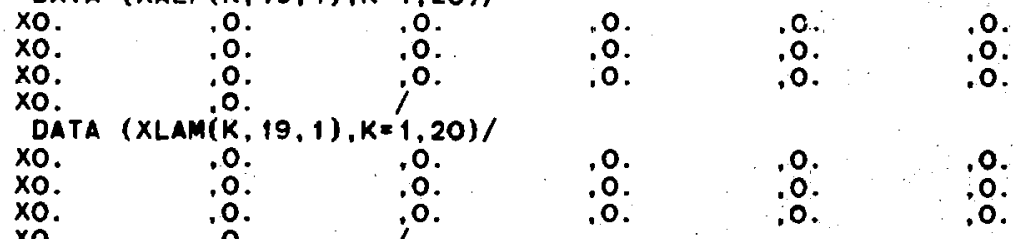

0

$i^{\circ}$

. 0 .

0

DATA 1740

DATA 1750

DATA 1760

DATA 1770

DATA 1780

DATA 1790

DATA1800

DATA 1810

DATA 820

DATA 1830

DATA 1840

DATA 1850

DATA 1860

DATA1870

DATA 1880

DATA 1890

DATA 1900

DATA 1905

DATA1910 
$X .1228 E-12, .4447 E-12, .9405 E-11, .3022 E-09, .6528 E-08, .3318 E-07$, DATA1920 $X .9476 E-06, .7533 E-05, .6507 E-04, .4 B 18 E-03, .8863 E-03,-.2132 E-03$, DATA1930 $X-.3858 E-05,-.3226 E-07, .4742 E-07,0 . \quad .0 . \quad 0 . \quad$,DATA 1940 Xo. $0 . \quad$ DATA1950 DATA (XLAM(K, 1,2),K=1,20)/ DATA 1960 $X .8579 E-09, .1275 E-07, .3172 E-07, .3403 E-06, .1314 E-05, .5054 E-05, D A T A 1970$ $X .3283 E-03, .1426 E-02, .1041 E-01, .4514 E-01, .3187 E+00, .4561 E-01.0 A T A 1980$ $X .1425 E-02, .6742 E-05 ; .3695 E-04,0.0 .0 . \quad$,DATA 1990 Xo. 0 .

DATA2000

DATA2010

DATA (XALP(K, 2,2$), K=1,20) /$

$X .4090 E-13, .2485 E-11, .2304 E-10, .1295 E-08, .2774 E-07, .4529 E-07.0 A T A 2020$ $X .6728 E-06, .5744 E-05, .9158 E-05, .1127 E-04, .1192 E-03, .7323 E-03$, DATA 2030 $X .1619 E-02, .9440 E-02, .2279 E-03,-.42 B 8 E-08,0 . \quad 0 . \quad$ DATA2O4O xo. 0 . i

DATA2050

DATA (XLAM(K, 2,2),K=4,20)/
$X .4802 E-03, .2080 E-07, .3117 E-07, .2722 E-06, .2963 E-05, .1461 E-04$. DATA2O60

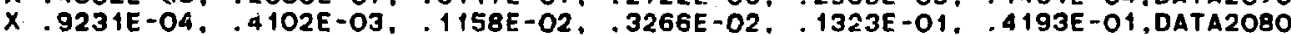
$X .1259 E+00 ; .7269 E+00 ; .1045 E+02, .4334 E-05.0$.
$X 0$. Xo.

DATA (XALP(K, 3, 2),K=1,20)/
$X .4262 E-14, .1991 E-13, .3188 E-15, .6465 E-11, .5067 E-08, .1193 E-07$. DATA2120 $X .5075 E-07, .1227 E-06, .1918 E-06, .1984 E-04, .5503 E-04, .1187 E-03.0 A T A 2130$ $\mathrm{X} .9778 E-03,0.3131 E-02 ; .1475 E-01, .2059 E-03,-.757 .4 E-02, .1270 E-01$.DATA2 140
$\mathrm{X} 0$. Xo. 0 .

DATA (KLAM(K, 3,2), K=1,20)/

DATA2 160

$X .6144 E-08, .9623 E-08, .1542 E-06, .1262 E-06, .6485 E-06, .1179 E-05.0 A T A 2170$ $X .3726 E-05, .2662 E-04, .1312 E-04, .3288 E-03, .1102 E-02, .7011 E-02,0 A T A 2180$ $X .1899 E-01 ; .9157 E-01 ; .3912 E+00, .1463 E+02, .4043 E+00 . .1026 E+01$. DATA2190 xo. 0 .

DATA2200

DATA (XALPIK, 4,2),K=1,20)/

DATA2210

$X .2064 E-13, .8636 E-12, .9578 E-11, .3139 E-08, .3769 E-07, .5032 E-06$. DATA2220

$X .1039 E-05, .2068 E-04, .8651 E-04, .1204 E-02, .7462 E-02, .1001 E+00$. DATA2230

$X-8124 E-01, .1455 E+00,-.1232 E+00 .-.1243 E-07.0 . \quad$. $0 . \quad$.DATA2240

Xo. $0 . \quad$ DATA2250

DATA (XLAMIK, 4,2), K=1,20)/

$X .2121 E-08,9932 E-08, .2363 E-07, .2110 E-06, .7255 E-06, .1045 E-04$ DATA2270

$X .4414 E-04, .2598 E-03, .1443 E-02,$. S3SE-01, .7951E-01, .4185E+00.DATA2280

$X .4654 E+00, .2092 E+01, .2209 E+01, .7486 E-06.0 . \quad 0 . \quad$.DATA2290

Xo. $0 . \quad$. DATA230O

DATA (XALP(K, 5.2), K=1,20) DATA2310

$X .2574 E-10, .7418 E-11, .2867 E-10, .1984 E-07, .2611 E-06, .1350 E-05$, DATA2320

$X .1972 E-04, .7089 E-04, .3109 E-03, .2301 E-02, .8318 E-02, .185$ 1E +01. DATA2330

$X-.1908 E+01, .1591 E+00,-.6489 E-01 ; .1156 E-07,0 . \quad 0 . \quad$.DATA2340

Xo. $0 . \quad$ OATA235O

DATA ( $X L A M(K, 5,2), K=1,20) /$

DATA2360

$X .7376 E-09, .1713 E-07 . .487$ IE-07, . 1228E-06, . 2894E-05, . 1485E-04.DATA2370

$\chi .1964 E-03, .1152 E-02, .4265 E-02, .2561 E-01, .1481 E+00 . .1395 E+01$. DATA2380

$X .1437 E+01, .3249 E+01, .4622 E+01, .1979 E-06,0 . \quad 0 . \quad$ DATA2390

Xo. 0 . $\%$ DATA2400

DATA (XALP(K, 6,2$), K=1,20) /$

DATA2410

$X .1259 E-16, .2938 E-12, .9255 E-13, .7414 E-08, .1273 E-07, .4374 E-07.0 A T A 2420$

$X .1052 E-05, .2150 E-04, .2559 E-04, .2235 E-03, .8837 E-03 . .34$ 1OE-O2, DATA2430

$X .1101 E-01, .4631 E-01, .2531 E-01.0 .0 .0 .0 .04 T 2440$

Xo. 0.0 DATA2450

DATA ( $X$ LAM(K, 6,2$), K=1,20) /$ DATA2460

$X .5695 E-09, .2122 E-07 . .3302 E-07, .5605 E-06, .8313 E-06, .4035 E-05.04 T A 2470$

$X .3179 E-04, .1608 E-03, .2116 E-03, .1521 E-02, .1108 E-0 ;, .4970 E-01.0 A T A 2480$

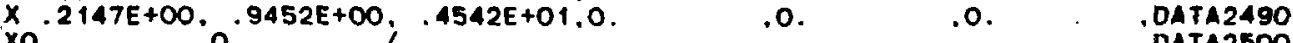

Xo. 0 . 0 DATA250O

DírA 'xalo(K, 7,2), K=1,20)/ DATA25 10

X.9554E-17. . 18Uut-11,.2464E-13. .1910E-10. .3362E-09. . 1093E-07.DATA2520

$X .2442 E-06 . .7977 E-06, .1458 E-04, .1037 E-03, .397$ IE-03,.2181E-02.0ATA2530

$X .5714 E-02, .1870 E-01, .3736 E-01,, .6221 E-01, .4891 E-01.0 . \quad .0 A T A 2540$

xo.

0 .

DATA2550 
DATA $(X \operatorname{LAM}(K, 7,2), K=1,20) /$

DATA.2560

$X .7047 E-09, .2167 E-07, .2207 E-07, .3623 E-06, .8302 E-06, .28$ 15E-05. DATA2570

$\mathrm{X} .1497 \mathrm{E}-04, .2924 \mathrm{E}-04, .1891 \mathrm{E}-03, .1162 \mathrm{E}-02, .6912 \mathrm{E}-02, .2700 \mathrm{0}-01, \mathrm{DATA2580}$ $X .1068 E+00, .4804 E+00, .1129 E+01, .2848 E+01, .4440 E+01,0$. ,DATA2590 XO. 0

$$
k=1,20) /
$$

DATA2600

DATA (XALP(K, 8,2$), K=1,20) /$

$X .2433 E-16, .3457 E-13, .1720 E-12, .3215 E-10, .3094 E-08, .2218 E-07.0 A T A 2620$

$X .8842 E-07, .3442 E-05, .1351 E-04, .1040 E-03, .2014 E-02, .7000 E-02,0 A T A 2630$

$X .1128 E+00 .-.3390 E-01 ; .5434 E+00 .-5846 E+00 .-1887 E-03,0 . \quad$.0ATA264O

$X 0.1128 E+00,0.3390 E-01, .5434 E+00 .-5846 E+00 .-.1887$

DATA (XLAM(K, $B, 2), K=1,20) /$

DATA2660

$X .1824 E-08, .2029 E-07, .3403 E-07, .1420 E-06, .1775 E-05, .2891 E-05 . D A T A 2670$

$X .1268 E-04, .5066 E-04, .2003 E-03, .1186 E-02, .1179 E-01, .9864 E-01.0 A T A 2680$

$X .8032 E+00, .7183 E+00, .2027 E+01, .1897 E+01, .1082 E-01.0 . \quad$ DATA2690

Xo.

DATA (XALP $(K, 9,2), K=1,20) /$

DATA2700

DATA2710

$X .2657 E-17, .4743 E-11, .2442 E-11, .1499 E-06, .1019 E-04, .1913 E-03,04 T A 2720$

$x, 1784 E-02, .8929 E-02, .1433 E-01,-.6409 E-02, .8391 E-02,-.2153 E-03,0 A T A 2730$

$X .1150 E-03,-.2028 E-06, .1274 E-06,-.1911 E-06, .7048 E-06,0 . \quad$ DATA2740

DATA (XLAM(K, 9,2$), K=1,20) / \quad$ DATA2760

$X .4328 E-09, .2620 E-07, .3741 E-07, .6916 E-06, .1507 E-03, .6732 E-03.04 T A 2770$

$X .1162 E-01, .2082 E+00, .1520 E+01, .3379 E-01, .3717 E-01, .8070 E-03, D A T A 2780$

$X .1756 E-02, .8980 E-06, .1065 E-05, .8004 E-05, .2568 E-04,0$, ,DATA2790

XO. 0 . 0 DATA28OO

DATA (XALP $(K, 10,2), K=1,20) / \quad$ DATA28 10

$X .2671 E-18, .1056 E-12, .4417 E-13, .1072 E-11, .1159 E-08, .1388 E-08.04 T A 2820$

$X .4823 E-06, .3281 E-05, .1291 E-04, .4864 E-03, .3784 E-02, .1598 E-01$. DATA283O

$X-.1040 E+00, .1049 E+00,-.2317 E-05,-.4039 E-03, .5889 E-03, .2100 E-02,041 \wedge 2840$

Xo. $0 . \quad$ DATA2850

DATA $(X \operatorname{LAM}(K, 10,2), K=1,20) /$

DATA2860

$X .6182 E-10, .2175 E-07, .2117 E-06, .8332 E-06, .2418 E-05, .5228 E-05 . D A T A 2870$

$X .2323 E-04, .1063 E-03, .4402 E-03, .1501 E-02, .1246 E+00 ; .4091 E+00,0 A T A 2880$

$X .1520 E+01, .1693 E+01, .4745 E+02, .1485 E-02, .1012 E-01, .3991 E-01 . D A T A 2890$

$X 0$.
$X$.

DATA (XALP $(K, 11,2), K=1,20) /$ DATA2910

$X .2024 E-18, .5844 E-13, .6540 E-10, .1507 E-08, .2069 E-08, .2169 E-07.0 A T A 2920$

$X .2723 E-06, .2706 E-05, .1521 E-04, .5338 E-04, .1569 E-03, .6287 E-03$, DATA2930

$X .1160 E-02, .6638 E-02 ; .1869 E-01, .2948 E-01,0$.
$X 0$.

XO. DATA (XLAMI $(K, 11,2), K=1,20) /$

DATA2960

$X .9974 E-10, .2208 E-07, .5904 E-06, .2846 E-05, .2872 E-05, .2110 E-04$, DATA2970 $X .2734 E-04, .1085 E-03, .4563 E-03, .1452 E-02, .6788 E-02, .1403 E-01.0 A T A 2980$ $X .3474 E-01.01469 E+00 ; .6451 E+00$. $1739 E+01.0$. .0. DATA2990 XATA (XALPi $(K, 12,2), K=1,20) /$

DATA3OOO

DATA3010

$X .2671 E-12, .3418 E-10, .2562 E-08, .3248 E-09, .3678 E-06, .2663 E-05$, DATA3O2O $X .2568 E-04, .8103 E-04, .1192 E-02, .4033 E-02, .8527 E-02, .1476 E+00,04 T A 3030$

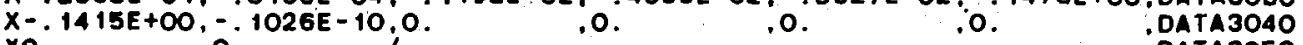
Xo. (

DATA (XLAM(K, 12,2),K=1,20)/ $X$.2200E-02,.1736E-01,.9531E-01,.3754E+00,. $4271 E+01$, DATA3080

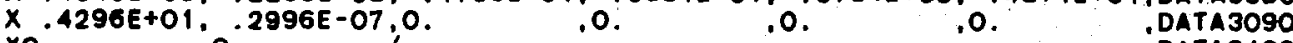
XO. O.<smiles>C1CCC(C2CCC2)C1</smiles>

DATA (XALP $(K, 13,2), K=1,20) /$ $X .8784 E-13,1050 E-16,1016 E-15,4116 E-11,9380 E-09,4627 E-09, D A T A 3120$ $X .1670 E-06, .2679 E-05, .1391 E-04, .6762 E-04, .1035 E-02, .5141 E-02$, DATA3130

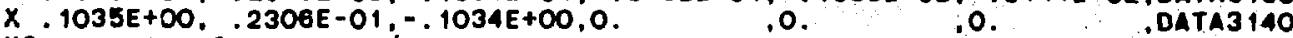
xo. 0 .

DATA3150 DATA3160

DATA (XLAM(K, 13,2), K=1,20)/

$X .2174 E-07, .1889 E-07, .3614 E-06, .4137 E-06, .6352 E-06, .2476 E-05$, DATA3170 $X .4117 E-04, .8088 E-04, .3708 E-03, .2403 E-02, .1483 E-01, .1085 E+00, D A T A 3180$ X.1296E+01,.3206E+01,.1609E+01,0. .0. .0. .DATA3190 
xo.

XO. DATA (XALPiK, 14,2$), K=1,20)$ )

DATA3200

DATA3210

$X .4550 E-13, .2803 E-14, .3774 E-09, .4319 E-08, .4902 E-06, .2073 E-05$. DATA3220

$X .5159 E-04, .1950 E-03, .1111 E-02, .3164 E-02, .6179 E-02, .1502 E-04$.OATA3230

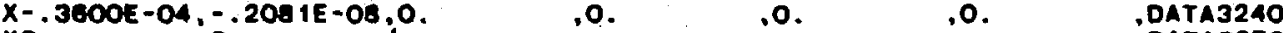
0 .

DATA3250

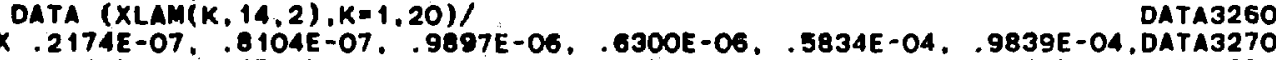

$x .0065 E-03, .4739 E-02, .2031 E-01, .9459 E-01 ; .3370 E+\infty 0 ; .1394 E+02$. DATA3280

$X .6874 E-03, .2182 E-05.0 . \quad$. $0 . \quad 0 . \quad .0 . \quad$.DATA3290

Xo. 0. DATA3300

DATA (XALP $(K, 15,2), K=1,20)$ )

DATA3310

$X .2495 E-13, .7280 E-14, .7767 E-10, .3281 E-06, .3060 E-05, .7557 E-05$, DATA3320

$X .1$ t6 1E-03,.1366E-02, 3349E-02, . 1577E-01, .1207E-02,-.3044E-01, DATA3330

$X .3383 E-01,-.5577 E-04 ; 0 . .0 .0 . .0 . . \quad$ DATA3340

xo. 0 .

DATA3350

DATA (XLAM(K, 15,2),K=1,20)/

DATA3360

$X .2173 E-07, .2794 E-07$. .6.198E-06. .5951E-04,.2018E-03, .3211E-03,DATA3370

$X .2357 E-02, .1367 E-01, .5757 E-01, .2826 E+\infty 0, .4177 E+01, .1392 E+01$, DATA3380

$x .1657 E+01, .3513 E+01,0 . \because 0 ., 0 ., 0 ., \quad$ DATA339O

xo. 0 . ,

DATA3400

DATA (XALP(K, 16,2),K=1,20)/

$x$.6504E-14, $2.669 E-18,5.395 E-13,4.068 E-11,2.719 E-07,3.819 E-06$. DATA3420

$X$ 5.467E-05, 2.832E-05, 1.781E-03, 5.359E-03, 1.624E-02, 2.948E-02,DATA3430

$X-1.603 E-02,7.184 E-03,-2.691 E-12,-3.153 E-11,0 . .0 \ldots$ DATA3440

xo. 0.

$f(20)$

DATA (XLAMIK, 16,2), K=1,20)/

DATA3460

$\times .2173 E-07,2.188 E-08,4.266 E-07,6.412 E-07,7.441 E-05,1.887 E-04$, DATA3470

$\times 1.244 E-03.1 .767 E-03,1.124 E-02$. 5.813E-02, 1.825E-01, 6.914E-01.DATA3480

$X 1.777 E+\infty 0,4.474 E+\infty 0,6.246 E-07,3.729 E-06,0 ., 0 ., \quad$ DATA349O

xo. 0 .

DATA3500

DATA (XALPiK, 17,2),K=1.20)/

DATA35 10

$X 2.168 E-19,6.995 E-09,2.267 E-08,1.791 E-07,5.588 E-05,2.432 E-04$.DATA3520

$X 1.063 E-03,6.520 E-03,2.135 E-02,3.497 E-03,-1.341 E-03,-6.313 E-09$, DATA3530

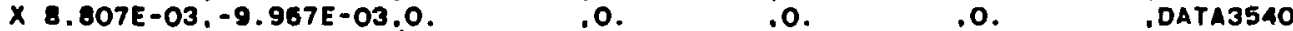

Xo. $0 . \quad$ DATA3550

DATA (XLAMY(K, 17,2), K=1,20)/ DATA3560

$X 4.141 E-06,6.319 E-05,7.671 E-05,4.034 E-04,3.650 E-03,5.320 E-03 . D A T A 3570$

$X 1.907 E-02,9.780 E-02,5.200 E-01,1.119 E+01,1.931 E-01,3.985 E-04 . D A T A 3580$

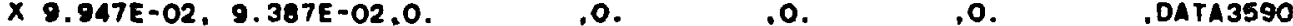

Xo. 0 . DATA3600

DATA (XALP(K, 18.2), K=1.20)/ DATA3610

$X 8.816 E-20,2.179 E-15,2.465 E-13,1.821 E-07,3.242 E-05,2.858 E-04$.DAT 43620

$X 2.039 E-01,-2.012 E-01,4.292 E-03,-2.908 E-03,9.703 E-04,-5.322 E-04, D A T A 3630$

$X-6.367 E-20,2.267 E-03,-1.939 E-03, .1000 E-20.0 . \quad$. $0 . \quad$.DATA3640

Xo. 0 . D DATA3650

DATA (XLAMIK, 18,2),K=1.20)/ DATA366O

$\times$ 5.758E-06, 2.011E-しd, 6.372E-04, 2.754E-03, 5.036E-03, 1.138E-02, DATA3670

$\times 2.014 E-01,2.022 E-01,4.014 E-01,4.045 E-01,1.201 E+00,1.824 E+00$.DATA3680

$\times 7.043 E-06,4.107 E-02,4.044 E-02, .1000 E-05,0 . \quad .0 . \quad$.DATA3690

Xo. 0.04 DATA370O

DATA $(X A L P(K, 19,2), K=1,20) /$

$\times 3.44040 E-21,8.39004 E-21,3.62256 E-19,4.63223 E-17,4.49234 E-9$.

$X 1.07130 E-5,1.31924 E-5,2.51500 E-4,-1.57334 E-4,8.27934 E-5$.

$X-1.63636 E-5,-9.44376 E-21,8 * 0.1$

DATA (XLAM(K, 19,2),K=1,20)i

$X$ 4.26703E-6, 2. $10226 \varepsilon-5,2.05656 E-4,6.05335 E-4,5.32009 E-3$.

$\times 8.59635 E-3,1.35035 E-2, .187459, .325807, .956266,1.13647$,

$\times 2,15828 E-5,8+0.1$

C FITS FOR PU-239 BETAS GROUPS 1 AND 2 COMBINED INTO 1

DATA3710

DATA3720

DATA3730

DATA3740

DATA3750

DATA3760

DATAB7ZO

DATA3780

DATA37QS

DATA (XALP(K,, 3$), K=1,20 \mathrm{~W}$, DATA3790

$X .3119 E-11, .5897 E-10, .7550 E-09, .3890 E-08, .3120 E-07, .0983 E-07$. DATA3800

$X .2280 E-06, .4645 E-05, .2900 E-04, .4929 E-04, .2540 E-03, .8475 E-03.0 A T A 3810$

$X .4021 E-04, .3211 E-04, .3215 E-05.0 . \quad$, $0 . \quad 0 . \quad$.DATA3B20 
xo.

( $\mathrm{K}$.

$k=1,20) /$

DATA3830

DATA (XLAM(K, 1,3) $K=1,20) /$ DATA3B4O

$X .8882 E-09, .4583 E-07, .8552 E-07, .2910 E-06, .1431 E-05, .6194 E-05.04 T A 3850$ $X .2573 E-04, .1800 E-03, .8218 E-03, .4258 E-02, .1513 E-01, .8165 E-01.0 A T A 3860$

$X .1433 E+00 . .4597 E+\infty 0 . .9689 E+01.0 . \quad 0.0$. .DATA3870 Xo. 0 .

DATA3880

DATA (XALP(K, 2.3) K=1.20)/.
$X .6815 E-11, .5074 E-40, .8639 E-09, .6740 E-08, .2546 E-07, .2057 E-06.0 A T A 3900$ $\mathrm{X} .1088 \mathrm{E}-05, .7335 \mathrm{E}-05, .6070 \mathrm{E}-04, .1277 \mathrm{E}-03, .5421 \mathrm{E}-03, .1033 E-02$, DATA3910 $X .8253 E-03, .5389 E-03, .6076 E-04,0 . \quad .0 . \quad 0 . \quad$.DATA3920 XO. 0 .

DATA3930

DATA (XLAM(K, 2,3$), K=1,20)$ /

DATA3940

$X .7591 E-09, .2618 E-07, .1405 E-06, .4525 E-06, .1392 E-05, .7443 E-05.0 A T A 3950$ $X .3189 E-04, .1980 E-03, .8686 E-03, .5066 E-02, .1791 E-01 ; .5746 E-01.0 A T A 3960$ $X .2753 E+\infty 0, .9173 E+00 ; .4599 E+01.0 .0 .0 .0$.DATA3970 Xo. $0 . \quad$ D DATA3980 DATA (XALP(K, 3,3),K=1,20)/

DATA3990

$X .2331 E-11, .6773 E-10, .4448 E-09, .6469 E-08, .2265 E-07, .1876 E-06.0 A T A 4000$ $X .9492 E-06, .2378 E-05, .2535 E-04, .8596 E-04, .4259 E-03, .1130 E-02.0 A T A 4010$ $\mathrm{X} .1605 \mathrm{E}-02 ; .2016 \mathrm{E}-02 ; .4068 \mathrm{E}-03.0$.
$\mathrm{X} 0$. $(K, 3,3), K=1,20) /$

DATA ( XLAMI $(K, 3,3), K=1,20) /$

DATA4040

$X .7847 E-09, .2413 E-07, .1174 E-06, .4460 E-06, .1391 E-05, .7015 E-05$, DATA4OSO $X .2461 E-04, .1012 E-03, .3937 E-03, .1494 E-02, .8519 E-02, .3219 E-01$. DATA4060 $X .8691 E-01,0.5182 E+00 ; .2517 E+01.0$.
$X 0$. $(K, 4,3), K=1,20)$

DATA (XALP (K, 4,3$), K=1,20) /$

DATA4090

$X .2049 E-11, .1108 E-09, .5264 E-09, .4655 E-08, .2002 E-07, .1850 E-06,0 A T A 4100$ $X .8825 E-06, .3569 E-05, .3391 E-04, .6498 E-04, .4854 E-03, .1538 E-02.0 A T A 4110$ $\mathrm{X} .3867 \mathrm{E}-02 ; .1255 \mathrm{E}-02 ; .7133 \mathrm{E}-05,0 ., 0 ., 0 .$, ,DATA4120 Xo. 0 .

DATA (XLAM(K, 4.3) $, K=1,20)$ ) 1156E-06,.4219E-06, . 1460E-05, .7192E-05.0ATA4150 $X .1339 E+000.4870 E-03, .1587 E-02, .7846 E-02, .2731 E-01$, DATA4160

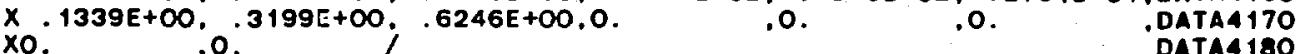

DATA (XALP'(K, 5.3),K=1,20)/

DATA4180

DATA4 190

$X .2439 E-11, .1568 E-09, .4441 E-09, .2337 E-08, .1279 E-07, .1047 E-06$. DATA4200

$X .4656 E-06, .2064 E-05, .2006 E-04, .7338 E-04, .5369 E-03, .1655 E-02$, DATA42 10

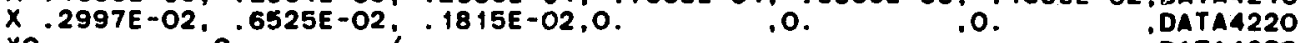
xo. 0

DATA (XLAM $(K, 5,3), K=1,20) /$

DATA4230

$X .7504 E-09, .2410 E-07, .1074 E-06, .3520 E-06, .1432 E-05,6781 E-05$ DATA4250

$X .1957 E-04, .7239 E-04, .3514 E-03 ; .1307 E-02$, .7554E-02,.2869E-01, DATA4260

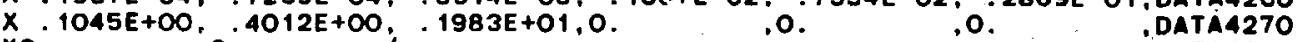

Xo. 0 .

DATA (XALP(K, 6,3), K=1,20)/ DATA4280 DATA4290

$X .2630 E-11, .2006 E-09, .2809 E-09, .1345 E-08, .6247 E-08, .4608 E-07.0 A T A 4300$ $X .3230 E-06, .1579 E-05, .1667 E-04, .6838 E-04, .5111 E-03, .1826 E-02$, DATA4310 $X .3938 E-02, .6417 E-02, .1507 E-02,0 . \quad 0.0$. .DATA4320 Xo.

DATA (XLAMí(K, 6,3$), K=1,20)$ )

DATA4330 DATA4340

$X .7524 E-09, .2402 E-07, .9519 E-07, .3138 E-06, .1343 E-05$, 6853E-05.DATA4350 $X .1819 E-04, .6169 E-04, .3381 E-03, .1311 E-02, .7685 E-02, .2827 E-01$.DATA4360 $X .1140 E+\infty, .4672 E+\infty 0, .2453 E+01,0,0.0$. .DATA4370 Xo. $\quad$ OATA4380 DATA (XALP(K, T,3),K=1.20)/ DATA4390 $X .2669 E-11, .2368 E-09, .1117 E-09, .6499 E-09, .3244 E-08, .2113 E-07$,DATA4AOO $X .2830 E-06, .1318 E-05, .1476 E-04, .6597 E-04, .4800 E-03, .1977 E-02$, DATA44 10 $X .5113 E-02, .8198 E-02 ; .1718 E-02.0$.
$\times 0$. XO. DATA (XLAM(K, 7,3$), K=1,20)$ $X .7529 E-09, .2395 E-07, .7533 E-07, .3016 E-06, .1357 E-05, .6895 E-05.0 A T A 4450$ $X .1905 E-04, .5995 E-04, .3314 E-03, .1306 E-02, .7605 E-02, .2859 E-01$.DATA4460 


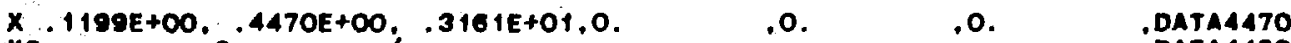

Xo. $0 . \quad$ DATA4480

DATA (XALP $(K, 8,3), K=1,20) / \quad$ DATA4490

$X .2420 E-11, .2604 E-09 . .37$ 15E-10,.3381E-09, . 1968E-08, . 1364E-07, DATA4500

$X .2439 E-06, .1116 E-05, .1347 E-04, .6335 E-04, .4102 E-03, .1905 E-02$. DATA45 10

$X .7426 E-02, .1102 E-01, .2431 E-02,0 ., 0 . \quad 0 . \quad$,DATA4520

Xo. 0 .

DATA (XLAM(K, 8,3), K=1,20)/ DATA4540

$X .7532 E-09 . .2386 E-07 . .5222 E-07, .3842 E-06, .1474 E-05, .7069 E-05.04 T A 4550$

$\mathrm{X} .1943 E-04 ; .5450 E-04 ; .3417 E-03 ; .1313 E-02 ; .7243 E-02, .2757 E-01.0 A T A 4560$

$X .1317 E+00, .5374 E+00, .1742 E+01.0, .0 .0 . \quad$ DATA4570

Xo.

DATA (XALP(K, 9,3), $K=1,20) /$ DATA4590

$X .1870 E-11, .2680 E-09, .3369 E-10, .1866 E-09, .1196 E-08, .6570 E-08,0 A T A 4600$

$X .1778 E-06, .8040 E-06, .1244 E-04, .6067 E-04, .3749 E-03, .1892 E-02.0 A T A 4610$

$\mathrm{X} .7177 \mathrm{E}-02,0.1117 \mathrm{E}-01 ; .2441 \mathrm{E}-02.0$.
$\mathrm{X0}$

DATA (XLAM(K, 9,3$), K=1,20) /$ DATA4640

$X .7537 E-09 . .2377 E-07 . .5089 E-07, .4034 E-06, .1600 E-05, .6887 E-05.0 A T A 4650$

$X .1972 E-04, .4704 E-04, .3563 E-03, .1310 E-02, .7270 E-02, .2882 E-01.0 A T A 4660$

$X .1275 E+00 ; .4606 E+00 ; .2457 E+01.0$.
$X 0$.

nATA (XALP $(K, 10,3), K=1,20) / \quad$ OATA4690

$X .1$ 15AE-11, .2582E-09, .3229E-10,.9044E-10, .5239E-09, . 3076E-OB, DATA4700

$X .16(14 E-06, .6059 E-06, .1017 E-04, .5633 E-04, .3146 E-03, .1642 E-02.04 T A 4710$

$x .78$ YOE-02,.1461E-0\%,.4786E-02.0. ,0. 0. .0ATA4720

Xo. 0 .

DATA (XLAM(K, 10,3), K= 1,20$) /$ DATA 1740

$X .7549 E-09, .2362 E-07, .4904 E-07, .3593 E-06, .1570 E-05, .6889 E-05$, DATA4750

$X .2091 E-04, .4810 E-04, .3897 E-03, .1238 E-02, .7345 E-02, .2806 E-01.04 T A 4760$

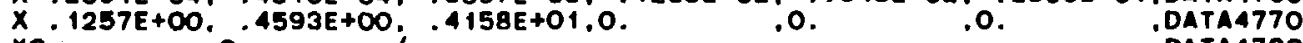

Xo. 0.0 DATA4780

DATA (XALP(K, 11,3),K=1,20)/

$X .4893 E-12, .2324 E-09, .2848 E-10, .3249 E-10, .1059 E-09, .2470 E-O B, D A T A 4800$

$X .1398 E-06, .4286 E-06, .9132 E-05, .5456 E-04, .2526 E-03 . .1547 E-02$. DATA48 10

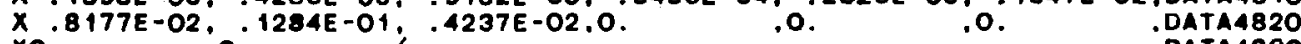

Xo. $0 . \quad$ DATA4830

DATA (XLAM(K, 11,3), K=1,20)/ DATA4840

$X .7587 E-09, .2344 E-07, .4668 E-07, .2829 E-06, .1664 E-05, .8128 E-05 . D A T A 4850$

$X .2152 E-04, .4695 E-04, .4246 E-03, .1236 E-02, .7192 E-02, .27 B 9 E-01$. DATA4860

$X .1323 E+\infty 0, .443 E+\infty 0, .4122 E+01,0 . \quad 0.0 . \quad$.DATA4870

Xo. $0 . \quad$ DATA4880

DATA (XALP $(K, 12,3), K=1,20) / \quad$ DATA4B9O

$X .1915 E-12, .1950 E-09, .2300 E-10, .2236 E-10, .2488 E-09, .3744 E-07$, DATA4900

$X .3702 E-08, .8964 E-05, .5010 E-04, .1958 E-03, .1476 E-02, .7887 E-02.0 A T A 4910$

$X .1339 E-01, .4395 E-02,0 . \quad .0 .0 . . \quad .0 . \quad$ DATA4920

Xo. 0.0 DATA4930

DATA (XLAM(K, 12,3),K=1.20)/ DATA4940

$X .7652 E-09, .2320 E-07, .5096 E-07, .3528 E-06, .3786 E-05 . .1540 E-04.04 T A 4950$

$X .3900 E-04, .4644 E-03 ; .1274 E-02, .6800 E-02, .2764 E-01, .1371 E+00.0 A T A 4960$

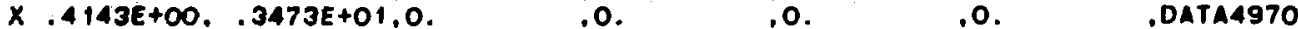

Xo. 0 .

DATA (XALP(K, 13,3),K=1,20)/ DATA4990

$X .1704 E-12$. 1474E-09. .1561E-10,.1592E-10,.1887E-09,.2041E-07.DATA5000 $X$.3010E-06,.9338E-05, .4301E-04,.1146E-03,.1512E-02,.7586E-02,DATA5010 $X .1418 E-01 . .4085 E-02.0 .0 .0 .0 .0 .04 T$. 0.020 Xo. $0.0 \%$ DATA5O3O DATA (XLAMIK, 13,3) $K=1,20) /$ DATA5040 $X .7606 E-09, .2289 E-07, .4910 E-07, .2551 E-06, .4225 E-05, .1492 E-04$, DATA5OSO $X .4040 E-04, .5009 E-03, .1338 E-02, .5592 E-02, .2749 E-01, .1422 E+00.0 A T A 5060$ $X .4999 E+00, .3028 E+01.0 .0 .0 .0 .0 .0 .040 .0075070$ Xo. (XALP(K, 14,3$), k=1,20) /$

$X .3008 E-12, .1551 E-09, .1037 E-10, .2433 E-10, .2786 E-09, .9032 E-08$, DATAS 100 
$X .4663 E-06, .1672 E-04, .6538 E-04, .2303 E-03, .2097 E-02, .1452 E-01$, DATA51 10

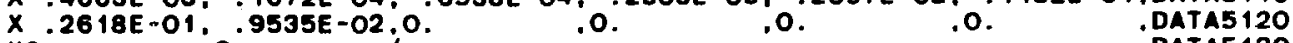
Xo.

DATA (XLAM(K, 14,3),K=1,20)/

DATA5130 UATAS 140

$X .7514 E-09, .2224 E-07, .4785 E-07, .2477 E-06, .4257 E-05, .1270 E-04$, DATA5 150 $X .4199 E-04, .5579 E-03, .1284 E-02, .6216 E-02, .2640 E-01, .1376 E+00$. DATA5 160

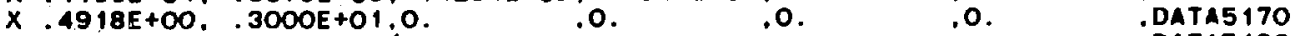
xo.

DATA (XALP $(K, 15,3), K=1,20) /$

DATA5 180

(XALP(K,15,3) $K=1,20) /$ DATA5 190

$X .4543 E-12, .3983 E-10, .8334 E-11, .2808 E-10, .1169 E-09, .6576 E-08$, DATA52OO

$X .5397 E-06, .2329 E-04, .8706 E-04, .4345 E-03, .3394 E-02, .3286 E-01$. DATA52 10

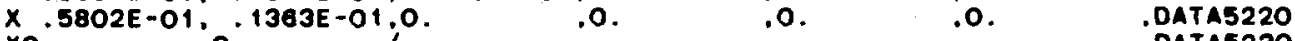

Xo: 0 .

DATA (XLAM(K, 15,3),K=1,20)/ DATA5240

$X .737$ TE-09, .212 TE-07, .9686E-07, .2722E-06, .3451E-05, . 1211E-04, DATA5250 $X .5192 E-04, .5555 E-03, .1414 E-02, .7911 E-02, .3107 E-01, .1565 E+00,0 A T A 5260$ $X .7539 E+00.01432 E+01.0 .0 .0 .0 .0 .00145270$ Xo. 0.0 DATA5280

DATA (XALP $(K, 16,3), K=1,20) /$

DATA5290

$x$ 1.453E-13,6.189E-12,6.941E-12,3.335E-10,3.960E-09, 2.7 16E-07, DATA53OO $X 1.927 E-06,4.636 E-06,7.133 E-05,5.462 E-04,1.437 E-02$, DATA5310 $\times 4.122 \mathrm{E}-02,1.510 \mathrm{E}-02,0.0 ., 0.0 .00 \%$ Xo. $0 . \quad ; \quad 0.0$ DATA5330

DATA (XLAM(K, 16,3), K=1,20)/

DATA5340

$\times 6.892 E-10,2.079 E-07,1.675 E-06,8.784 E-06,2.069 E-05,7.051 E-05.0 A T A 5350$ $\times 4.956 E-04,1.579 E-03,7.532 E-03,2.272 E-02,1.325 E-01,6.950 E-01$, DATA5360

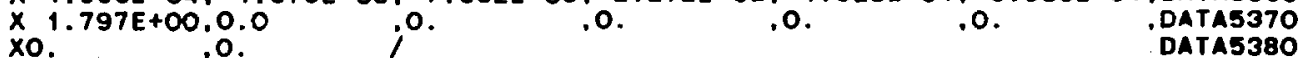

DATA (XALPI $K, 17,3), K=1,20) /$ DATA5390

$\times 3.722 E-14, B .313 E-13,4.292 E-10,1.992 E-08,1.150 E-06,2.139 E-05$, DATA5400 $\times 9.618 E-05,1.830 E-03,1.120 E-02,3.234 E-02,-1.431 E-02,7.555 E-03$, DATA54 10 $X-4.665 E-03,-1.395 E-08,-1.568 E-12,1.454 E-12.0$. . $0 . \quad$.DATA542O XO O. $1,20)$

DATA5440

DATA (XLAM(K,17,3),K=1,20)/

$\times 7.203 E-10,3.787 E-07,1.307 E-05,6.274 E-05,1.595 E-03,8.367 E-03.0 A T A 5450$

$\times 3.223 E-02,9.089 E-02,2.464 E-0,1.048 E+00,6.151 E-01,5.771 E+00.04 T A 5460$

$\times 2.811 E+00,6.510 E-05,5.143 E-0,0.850 E-07,0$. . 0.040

XO. DATA (XALP $(K, 18,3), K=1,20)$

$\times 2.02247 E-15,4.26636 E-13,8.99431 E-10,5.76412 E-8,2.47780 E-6$.

$\times$ 5.85628E-5, 1.0375 tE-3, 3. 19925E-3, $1.15261 E-2,-6.31111 E-3$.

DATAB480

MATA5490

DATA5EOO

$\times 2.854 \% 9 E-3,-1.53200 E-3,-9.53371 E-10,4.09241 E-10,-2.67547 E-13$. DATA55 10

$x \mathbf{5} \neq 0.1$

DATA ( $X$ LAM $(K, 18,3), K=1,20)$ )

$X 6.81882 E-10,6.30735 E-7,1.63083 E-5,1.52073 E-3,6.16627 E-3$,

$X 1.97103 E-2,7.39598 E-2,1.74214 E-1, .793894, .890645$

$X 2.87581,3.57001,1.75916 E-5,3.26377 E-5,6.3666 E-7,5 * 0 . /$ DATA (XALP(K, 19,3), K=1,20)/

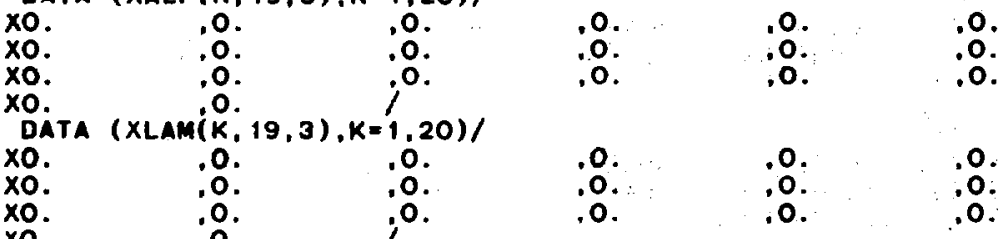

XO. FOR PU-239 GAMMAS GROUPS 1 THROUGH 19

DATA5520

DATA5530

DATA5540

DATA5550

DATA5560

DATA5570

DATA5580

DATA5590

DATA5600

DATAS6 10

DATA5620

DATA5630

DATA5640

DATA5650

Datas660

DATA5670

DATA5675

DATA (XALP $(K, 1,4), K=1,20) /$
$X .1675 E-12, .5868 E-12, .6533 E-11, .1563 E-09, .2942 E-08, .1025 E-07.0 A T A 5690$ $X .7436 E-08, .3483 E-07, .9955 E-06, .4669 E-05, .2706 E-04, .1908 E-03,0 A T A 5700$ $X .5654 E-03, .6856 E-01, .7029 E-01, .4682 E-02,-.2124 E-02,0 . \quad$.DATA5710 xo. 0 . $=1,20)$

DATA5720

DATA $(X \operatorname{LAn}(K, 1,4), K=1,20) /$

DATA5730 
$X .1053 E-08, .7491 E-08, .3193 E-07, .2533 E-06, .9501 E-06, .2326 E-05$. DATA574O $X .9554 E-05, .9956 E-04, .3260 E-03, .1420 E-02, .6438 E-02, .1786 E-01$, DATA5750 $X .9085 E-01, .1582 E+01 ; .1659 E+01, .4954 E+01 . .7501 E+01.0 . \quad$.DATA5760 Xo. DATA (XALP(K, 2,4$), K=1,20) /$ DATA5780 $X: 1895 E-12, .7624 E-12, .1603 E-10, .1104 E-08, .2778 E-07, .5213 E-07$, DATA5790 $X .7963 E-06, .5556 E-05, .1684 E-04, .8240 E-04, .8442 E-03, .2436 E-02$, DATA58OO $X .2005 E-01,-.8527 E-02 ;-.4437 E-08,0.0 .0 .000$ Xo. $0.1 \%$ DATA5820 DATA (XLAM(K, 2,4$), K=1,20) /$

$X$.457EE-08,. 1647E-07,.2997E-07,.2627E-06, .2903E-05, . 1534E-04, DATA5840 $X .1357 E-03, .4019 E-03, .1534 E-02, .7615 E-02, .4544 E-01, .2401 E+00$, DATA5850

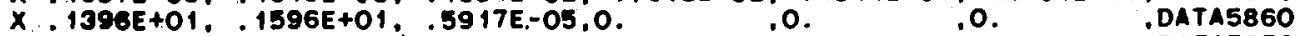
$\times 0$ . $=1.20) /$ DATA5B70 DATA (XALP(K, 3,4),K=1,20)/ DATA5880 $X .1904 E-13,8134 E-13,7531 E-13, .1019 E-10, .5421 E-08, .1798 E-07$. DATA5890 $X$.6339E-07,.3285E-06,.2898E-06,.2682E-04,.4978E-04,.5306E-03.DATA5900 $X .1899 E-01, .1015 E+00,-.1124 E+00,-.231$ 1E-01, .1205E-01. . 1644E-01. DATA5910 xo 0. $1,20)$

DATA5920 DATA5930

$X .6099 E-08,1012 E-07,3884 E-07, .1499 E-06, .6605 E-06, .1235 E-05$.DATA5940 $X .1014 E-05, .1366 E-04, .3175 E-04, .4061 E-03, .8420 E-03, .9422 E-02$, DATA5950 $X .8051 E-01, .7403 E+01, .6864 E+01, .9396 E-01, .1646 E+00, .2363 E+01$. DATA5960 xo. 0 . DATA5970 DATA (XALP $(K, 4,4), K=1.20) /$ DATA5980

$\times$ 158OE-12, 1981E-10,.1081E-09,.7883E-08,.3127E-07,.4183E-06.DATA5990 $X .1132 E-05, .1938 E-04, .4860 E-04, .1030 E-03, .5790 E-03, .6223 E-03$, DATA6O0O $\times$ 102BE-01, 3843E-01,-.1548E-01,.4805E-02,-.2382E-02,-.5746E-08,DATA6010 xo. 0 . 1 DATAGO2O

DATA ( $X L A M(K, 4,4), K=1,20) /$

DATAGO30

$X .4501 E-08, .1410 E-07, .2817 E-07, .2249 E-06, .8420 E-06, .1010 E-04$, DATA6O4O

$X$. AS6OE-04; .2835E-03,.943 TE-03;.3519E-02,. 1204E-01. .2756E-01.DATA6O5O $X .1147 E+\infty 0, .5050 E+00, .5633 E+00, .2563 E+01, .4641 E+01, .7773 E-06, D A T A 6060$ xo 0 . (.20) DATA6070

DATA (XALP(K, 5,4) K=1,20!/
X.2743E-10, . 1232E-10, .7873E-10. . 1462E-07. .3050E-06, .8594E-06.DATA6090 $X .2487 E-05 ; .2047 E-04 ; .3840 E-04, .1538 E-03, .4903 E-03, .2285 E-02 . D A T A 6100$ $X .1239 E-01, .5805 E-01,-.1241 E+\infty, .7718 E-01,-.4958 E-03,-.7299 E-08$, DATA6110

xo. 0 . i DATA6120

DATA (XLAM(K, 5,4$), K=1,20) /$

$X .7278 E-09, .1087 E-07, .2966 E-07, .1240 E-06, .2826 E-05, .1520 E-04 . D A T A 6140$ $X .6046 E-04, .2516 E-03, .9024 E-03, .2905 E-02, .1092 E-01, .4344 E-01,0 A T A 6150$ $x .2716 E+00 ; .2337 E+01 ; .4393 E+01 ; .5731 E+01, .2642 E+00, .1969 E-06$. DATA6160
$X_{0} .0$.

DATA (XALP(K, 6,4),K=1.20)/
$X .6121 E-15, .3415 E-11, .3061 E-11, .1473 E-07, .4877 E-07, .4367 E-06$, DATA6190

DATA (XALP(K, 6,4),K=1.20)/
$X .6121 E-15, .3415 E-11, .3061 E-11, .1473 E-07, .4877 E-07, .4367 E-06$, DATA6190 $X .2233 E-04, .3467 E-04, .1332 E-03, .3924 E-03, .7549 E-02, .2176 E-01$. DATA6200

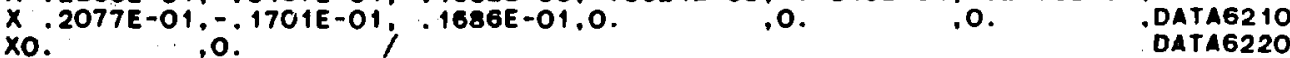

DATA (XLAM(K, 6,4$), K=1,20) /$

DATA6230

$X .2209 E-08, .2162 E-07, .1876 E-06, .6308 E-06, .2943 E-05, .2015 E-04$, DATA6240 $X .1508 E-03, .342$ IE-03,. $1381 E-02, .6926 E-02, .4757 E-01, .5772 E+00,0 A T A 6250$ $X .3649 E+01.0936 E-01 ; .9324 E-01.0 .0 .0 .00 \%$ DATA6270

DATA (XALP $(K, 7,4), K=1,20) /$ $X .3386 E-15, .1891 E-10, .1015 E-11, .5966 E-09, .1488 E-08, .1405 E-07 . D A T A 6290$ $X .9484 E-07, .9313 E-06, .1396 E-04, .7$ I8OE-04,.1907E-03,. 1327E-02.DATA6300 $X .4988 E-02, .2417 E-01,-.1155 E-01, .3024 E-02,-.6802 E-04.0 . \quad$.DATA6310 $\times 0$.

DATA (XLAMI $(K, 7,4), K=1,20)$ / DATA6320 DATA6330 $X .1872 E-08, .2159 E-07 . .3497 E-07, .5608 E-06, .1414 E-05, .3147 E-05$, DATA6340 $X .1136 E-04, .3355 E-04, .2250 E-03, .9838 E-03, .4337 E-02, .2133 E-01$, DATA6350 $\times .1000 E+00, .5331 E+00, .1364 E+01, .2902 E+01, .3011 E-01.0$. , DATA6360 xo. .0 .

DATA6370 
DATA (XALP(K, B, 4),K=1,20)/

DATA6380 $\mathrm{X} .2566 \mathrm{E}-07, .1673 \mathrm{E}-05, .9010 \mathrm{E}-05, .4957 \mathrm{E}-04, .1260 \mathrm{E}-03, .1326 \mathrm{G}-02.0 \mathrm{DATA6400}$ $X .2244 E-02, .9506 E-02, .1301 E-01, .1261 E-03,0 . \quad 0 . \quad$ DATAGA10

XO.

DATAG420

DATAG430

$X .2421 E-08, .1670 E-07, .3130 E-07, .1495 E-06, .6177 E-06, .2425 E-05.0 A T A 6440$ $x .5153 E-05, .4161 E-04, .1913 E-03, .7425 E-03, .2879 E-02, .1422 E-01.0 A T A 6450$ $\mathrm{X} .6297 E-01, .2560 E+00 ; .8232 E+00, .1514 E+02.0 . \quad .0 . \quad$.0ATA6460 xo.

DATAG470

DATA (XALP(K, 9,4$), K=1,20) /$

DATA6480

$X .6011 E-16, .3490 E-11, .3876 E-11, .6253 E-0 B,, 7373 E-04,, 1356 E-02$, DATA649N $\mathrm{X} .1885 E-02,6279 E-02, .1381 E-01,-.7309 E-02, .2441 E-02,-.4071 E-02.0 A T A 6500$ $X-.2959 E-04, .1626 E-03,-.1617 E-05, .9953 E-06, .0030 E-06, .2093 E-06$, DATAG5 10 $X-.1985 E-06,0$.

DATA $(X L A M(K, 9,4), K=1,20)$ (

DATA6520

$X .1414 E-08, .2207 E-07, .3063 E-07, .7440 E-06, .3949 E-03, .1522 E-01$, DATA6540

$X .7589 E-01, .2278 E+00, .7323 E+00, .5955 E+00, .1533 E+01, .2331 E+01,0 A T A 6550$

$X .4123 E-03, .3979 E-02, .8158 E-06, .8456 E-06, .4587 E-04, .1344 E-05.0 A T A 6560$

$\mathrm{X} .1894 \mathrm{E}-0 \mathrm{5}, 0$.

DATA (XALP $(K, 10,4), K=1,20) /$

DATA6570

$X .1420 E-17, .1126 E-11, .2791 E-12, .8436 E-11, .1764 E-08, .2082 E-07.0 A T A 6590$

$X .4911 E-06, .4814 E-05, .1441 E-04, .5855 E-04, .2359 E-03, .1169 E-02.0 A T A 6600$

$X .1270 E-01,-.8152 E-02, .8864 E-02,-.5294 E-02, .2934 E-02,-.1143 E-02$. DATA66 10

Xo: 0 .

DATA6620

DATA (XLAM $(K, 10,4), K=1,20) /$

DATA6830

$X .1549 E-09, .2180 E-07 . .2003 E-06, .7512 E-06, .2556 E-05, .1121 E-04$. DATA6640

$X .2763 E-04, .1758 E-03, .5645 E-03, .1182 E-02, .7098 E-02, .2469 E-01$. DATA6650

$X .1117 E+00, .1193 E+00, .3794 E+C O, .1297 E+01, .4467 E+01, .3307 E+01$, DATA6660

xo. 0 .

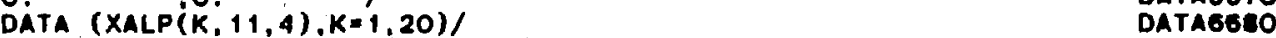

DATA6670

$X .9696 E-18, .6832 E-12, .1720 E-09, .3996 E-08, .4361 E-07, .6644 E-06.0 A T A 6690$

$X .7149 E-05, .4754 E-04, .1041 E-03, .7792 E-03, .1092 E-02, .4851 E-02$, DATA6700

$x .1016 E-01 ; 0.0740 E-02 ; .3880 E-02.0$
$X 0$.

DATA (XLAM(K, 11,4),K=1,20)/ DATA6730

$X .9565 E-10, .2180 E-07, .5658 E-06, .2702 E-05, .1756 E-04, .6416 E-04.0 A T A 6740$ $X .2874 E-03, .1009 E-02, .4623 E-02, .1667 E-01, .3861 E-01, .1740 E+00, D A T A 6750$ $\mathrm{X} .5496 \mathrm{E}+00 ; 0.1163 E+01 ; .2173 E+01.0$.
$\mathrm{X} 0$.

DATA (XALP' $K, 12,4), K=1,20) i$

.9985E-10,.5056E-11,. 3573E-09, DATA6790

$X .3964 E-06, .9300 E-05, .4799 E-04, .1216 E-03, .1386 E-02, .5089 E-02.0 A T A 6800$ $X .3932 E-02, .4930 E-02,-.6688 E-02,-.3144 E-05,0 \%$, $0 .$, DATAGE 10 Xo. $0 . \quad$ DATAG820

DATA (XLAM $(K, 12,4), K=1,20) /$ DATAGE30

$X .2465 E-07, .3071 E-07, .9914 E-07, .3122 E-06, .1260 E-05, .2072 E-05,0 A T A 6040$ $X .5483 E-04, .2280 E-03, .8095 E-03, .4200 E-02, .2219 E-01, .1682 E+00.0 A T A 6530$ $X .5132 E+00, .3755 E+01, .2049 E+01, .1940 E-03.0$. . 0. .DATAGESO Xo. 0 . $\quad$ DATAGOTO

DATA (XALP(K, 13,4),K=1.20)/ DATABSEO

$X .9396 E-12, .2560 E-15, .1045 E-11, .8002 E-09, .2344 E-09, .1830 E-08$.DATA6B90 $X .7367 E-06, .9824 E-05, .3303 E-04, .2582 E-03, .1420 E-02, .5593 E-02.0 A T A 6900$ $X .4160 E-02, .4263 E-02,-.5712 E-02,-.4260 E-05,0 ., 0 .$, DATAG910 Xo. $0 . \quad j$ DATAG920 DATA ( $X L A M(K, 13,4), K=1,20) / \quad$ DATAG930 $X .2179 E-07, .3071 E-07, .3544 E-06, .6192 E-06, .1211 E-05, .5411 E-05.04 T A 6940$ $\mathrm{X} .5950 E-04 ; .2018 E-03 ; .9124 E-03, .7439 E-02, .2534 E-01, .2142 E+00$. DATAG950 $X .6752 E+00, .4459 E+01, .2545 E+01, .1683 E-03,0, .0 . \quad$ DATA6960 XO. 0 .

DATA (XALP(K, 14,4),K=1,20)/

DATAG970

$X \quad-4848 E-12,0,1252 E-14,0.36, x$

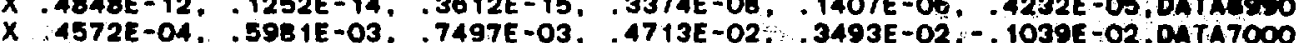
X .6771E-03,-.5645E-03,-. 1469E-04, . I990E-06,-.2004E-06.0. ,DATA7010 
xo.

0 .

DATA7020

DATA (XLAM(K.14,4),K=1,20)/ DATA7030

$X .2178 E-0 ?, 2263 E-07, .2264 E-07, .6139 E-06, .4253 E-04, .1602 E-03$. DATA7040

$X .8998 E-03, .1206 E-01, .4243 E-01, .2018 E+\infty 0, .7063 E+\infty 0 . .1277 E+01$. DATA7050

$X .2275 E+01, .3738 E+01, .7934 E-03, .1692 E-05, .1705 E-05.0 . \quad$.DATA7000

xo 0.

DATA7070

DATA (XALP $(K, 15,4), K=1,20)$ )

DATA7080

$X .2680 E-12,4346 E-14, .7312 E-10, .2684 E-12, .6776 E-03, .1388 E-05$, DATA7090

$X$.68B6E-05, .4367E-04, . 107OE-01, - . 1098E-01, .2709E-02, . S04OE-02, DATA7 100

$X .1087 E-01,-.5809 E-02, .1904 E-03,0.0 ., 0 . \quad$.DATA71 10

xo.

0

DATA7130

DATA ( $X L A M(K, 15,4), K=1,20) /$

DATA7 130

$\times$ 2170E-07, 2553E-07,6240E-06, 6835E-05, 1993E-04, $1158 E-03, D A T A 7140$

$X .3099 E-03, .1240 E-02, .1038 E-01, .1074 E-01, .2116 E-01$. 1682E+00. DATA7 150

$X .5767 E+\infty, .1301 E+01, .1162 E+02,0.0 .0 .0 .0 .0407160$

XO. $0 . \quad / \quad$ DATA7170

DATA (XALPi $K, 16,4), K=1,20) /$

DATA7180

$X .6978 E-13, .6465 E-16, .3321 E-10, .1827 E-06, .1733 E-05, .3355 E-04.0 A T A 7190$

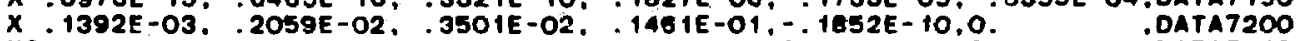

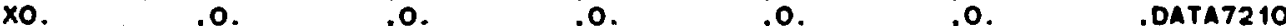

Xo. $0 . \quad \%$ Data7220

DATA (XLAM(K, 16,4),K=1,20)/

DATA7230

$X .2179 E-07, .3507 E-07, .6308 E-06, .9524 E-04, .1474 E-03, .7336 E-03,04 T A 7240$

$X: 3629 E-02 ; .1808 E-01 ; .8523 E-01 ; .2323 E+00 ; .3354 E-05.0 . \quad$.0ATA7250

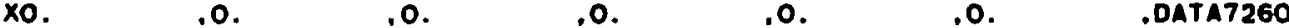

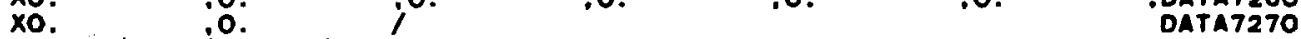

DATA (XALP $(K, 17,4), K=1,20) /$

$\times 7.781 E-19,8.311 E-09,2.357 E-08,1.956 E-07,1.681 E-06,1.591 E-04.0 A T A 7290$

$\times 7.875 E-04,1.763 E-03,4.693 E-03,4.702 E-05,2.463 E-05,0.0$.0ATA7300

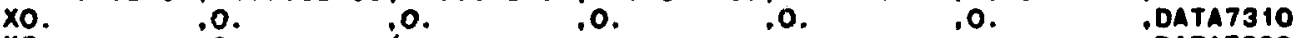

Xo. $\quad 0$. $\quad ; \quad$ DATA7320

DATA (XLAM(K, 17,4),K=1,20)/

$\times$ 4.142E-06. 6.800E-05, 1.478E-04, 6.896E-04, 5.208E-03, 5.207E-03.DATA7340

$\times 2.489 E-02,1.236 E-01, .2862 E+00, .9943 E+00, .1620 E+02.0 . \quad$ DATA7350

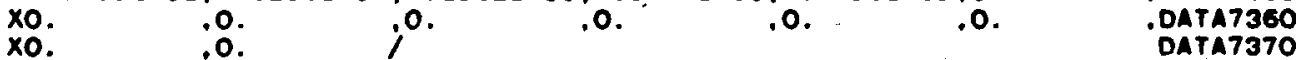

DATA (XALP $(K, 18,4), K=1,20) /$

$X$ 1.200E-19,3.004E-15,1.191E-12,2.959E-7,2.493E-5,2.012E-4,

$\times$ 9.907E-6.1.325E-3,1.418E-3.-1.811E-20.0.0.0.0.

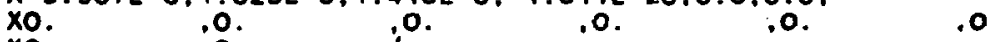

Xo. 0 .

DATA7380

DATA7390

DATA7400

DA7A $(X L A K(K, 18,4), K=1,20) /$

X 4.209E-06, \&. 022E-04, 6.552E-04, 3.008E-03, 6.336E-03, $1.921 E$ 02 nATA7440

$X 3.258 E-02,1.539 E-01,9.212 E-01,5.945 E-06,0.0 \quad 0.0$.DATA7450

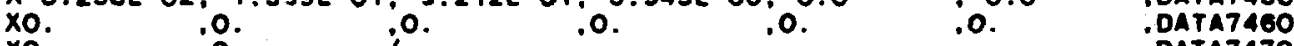

Xo.

DATA (XALP $(K, 19,4), K=1,20) /$

$\times 1.85545 E-20,3.305$ 19E-17,9,72023E-16,3,86898E-6, 1.02786E-5.

$x \quad 23228 E-4,-4.42133 E-5,2.57210 E-5,-6.98988 E-6,-2.50522 E-6$,

$x-7.29476 E-21.2 .15247 E-20,8 * 0.1$

DATA (XLAM(K, 19,4),K=1,20)/

$\times 4.57245 E-6,4.41793 E-4,8.97811 E-4,8.03833 E-3,1.22545 E-2$.

$\times 1.90914 E-1,2,53015 E-1,1,11151,2.78967,1.33733 E-2$.

$\times 6.83542 E-6,6.22339 E-3.8 * 0.1$

DATA KTRM/

$x 18,19,18,19,17,18,19,18,15,15,16,16,15,17,17,15,15,13,0,15,16$,

$\times 18,16,16,15,17,17,17,16,18,14,15,14,14,16,14,16,12,15,15,15,15$,

$\times 15,15,15,15,15,15,15,14,14,13,14,13,18,15,0,17,15,18,18,18,15$

$\times 17,16,19,18,15,16,16,17,15,11,11,10,121$

ten

SUDNOUTINE SETUP (X1,Y1,L1,TL,NT, XL, NX, YL, NY, ITYPE, IMK, ICON, IGRD) REAL XI(LI), Y I LI

INTEOER TL(2), XL(2), YL(2)

DATS XPAGE, YPAOE /6.,6.J

DATA 7480

DATA7490

DATA7500

DATA75 10

DATA7520

DATA7530

DATA7540

DATA7550

DATA7560

DATA7570

DATA7SEO

DATA7590

DATA7600

DATA7610

SETU 10

SETU 20

SETU 30

SETU 10 
CALL BGNPL (-1)

CALL NOBRDR

CALl PAGE (XPAGe, YPAGE)

$X G=X P A G E-1.7$

YG $=Y P A G E-1.7$

HITE $=.03 * Y G$

CALL HEIGHT (HITE)

CALL SCMPLX

CALL MX 1ALF (5HSTAND, 1H>)

CALL MX2ALF (5HL/CST, 1H<)

CALL MX3ALF (5HINSTR, 1HW)

$X L E F T=X R I G H T=X 1(1)$

$Y T O P=Y B O T=Y 1(1)$

DO $5 \quad I=2, L 1$

IF $(X I(I)$.LT.XLEFT) XLEFT $=X 1(I)$

IF $(X 1(I) . G T . X R I G H T) \quad X R I G H T=X 1(I)$

IF.(YI I ).LT.YBOT) YBOT=YI(I)

5

IF(YT(I) GT YTOP) YTOP=YI(I)

CONT INUE

IF (ITYPE,LT.3) GO TO 6

TOP $=A L O G I O$ (XRIEHT)

IF (TOP.GE.0.) XRIGHT =10.*\#IFIX(TOP+.99)

IF (TOP.LT.O. ) XRIGHT $=10 . * * I F I X(T O P)$

IF(XLEFT.NE.O.O) GO TO 6

$X L E F T=10$ * * IFIX(TOP-15

6

IF (ITYPE.NE.2.AND. ITYPE. NE.4) GO TO 7

$T O P=A L O G I O(Y T O P)$

IF (TOP.GE.O.) YTOP=10.*\#IFIX(TOP+.99)

IF (TOP.LT..O.) YTOP $=10 . *$ IFIX (TOP)

IF (YBOT . GT:0.0) CO TO 7

YBOT $\approx 10 . *$ IF IX (TOP-15.)

7 IF (ITYPE.NE . 1) GO TO 10

CALL AXSPLT (YBOT, YTOP, YG, YORIG, YSTEP, YAXIS)

$Y T O P=I F I X($ (YTOP+YSTEP )/YSTEP ) * YSTEP

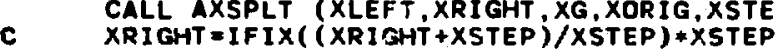

CALL TITLE $(0,0, X L, N X, Y L, N Y, X G, Y G)$

CALL GRAF (XORIG,XSTEP, XRIGHT, YORIG, YSTEP, YTOP) GO TO 40

10 IF (ITYPE.NE. 2) GO TO 20

CALL AXSPLT (XLEFT, XRIGHT XG, XORIG, XSTEP, XAXIS)

XRI CHT = IF IX ( (XRIGHT+XSTEP)/XSTEP ) *XSTEP

CALL ALGPLT (YBOT, YTOP, YG, YORIG, YCYCLE)

CALL TITLE $(0,0,0,0, Y L, N Y, X G, Y G)$

CALL YLOG (0.,1., YORIG, YCYCLE)

CALL XGRAXS (XORIG, XSTEP, XRIGHT, XG, XL,NX,O, O.) CO TO 40

20 CALL ALGPLT (XLEFT, XRIGHT, XG, XORIG, XCYCLE)

IF (ITYPE.NE.3) GO TO 30

CALL AXSPLT (YBOT, YTOP, YG, YORIG, YSTEP, YAXIS)

$Y T O P=I F I X($ ( YTOP+YSTEP $) / Y S T E P) * Y S T E P$

CALL TITLE (0,0.XL,NX,0,0,XG,YG)

CALL XLOG (XORIG,XCYCLE.O. 1.)

CALL YGRAXS (YORIG,YSTEP, YTOP, YG, YL,NY,O, O.) GO TO 40

30 CALL ALGPLT (YBOT, YTOP, YG, YORIG, YCYCLE)

CALL TITLE (O,O,XL,NX,YL,NY,XG,YG)

CALL LOGLOG (XORIG, XCYCLE, YORIG, YCYCLE)

10 CALL FRAME

IF (IGRD.EO. 1) CALL GRID $(1,1)$

IF ( ICRD.NE. 2 ) GO TO 41

CALL BLNK1(. 13,XG-.13,0., YG,0)

CALL GRID $(0,1)$

CALL RESET(SHBLAK1)

CALL BLNK1(0.,XG,.13,YG-.13,0)

CALL GRID $(1,0)$

CALL RESET (SHBLNK1)

41 IF (ICON. NE. O) CALL MARKER (IMK)

CALL CURVE (X1,Y $1, L 1, I$ CON)

IF (NT .GT . O)CALL MESSÁG(TL,NT, - S, YPAGE-1.)

RETURN

END

SETU 50

SETU 60

SETU 70

SETU 80

SETU 90

SETU 100

SETU 110

SETU 120

SETU 130

SETU 140

SETU 150

SETU 160

SETU 170

SETU 180

SETU 190

SETU 200

SETU 210

SETU 220

SETU 230

SETU 240

SETU 250

SETU 260

SETU 270

SETU 230

SETU 290

SETU 300

SETU 310

SETU 320

SETU 330

SETU 340

SETU 350

SETII 360

SETU 370

SETU 380

SETU 390

SETU 100

SETU 410

SETU 420

SETU 430

SETU 440

SETU 450

SETU 460

SETU 470

SETU 480

SETU 490

SETU 500

SETU 510

SETU 520

SETU 530

SETU 540

SETU 550

SETU 560

SETU 570

SETU 580

SETU 590

SETU 600

SETU 610

SETU 620

SETU 630

SETU 640

SETU 650

SETU 660

SETU 670

SETU 680

SETU 690

SETU 700

SETU 710

SETU 720

SETU 730

SETU 740

SETU 750

SETU 760 
SAMPLE PROBLEM INPUT

$75,1, E+14,5 . E+14,7,2 E+7,-12,2,0,0$

1. E+6.1.E+8

$1,1,1 \ldots 1$.

SAMPLE PROBLEM OUTPUT

FISSION PRODUCT DECAY ENERGY FOR A MIXTURE OF U-235 AND PU-239

PERCENT U-235

PERCENT PU-239

7. $5000 E+01$

THERMAL FLUX

2. $5000 E+01$

EP I THERMAL FLUX

OPERAT ING TIME

COOLING TIME

o

$\begin{array}{rcc}\text { GRP } & \begin{array}{c}\text { ELO } \\ \text { (MEV) }\end{array} & \begin{array}{c}\text { EHI } \\ \text { (MEV) }\end{array} \\ 1 & 0.0 & .4 \\ 2 & .4 & .8 \\ 3 & .8 & 1.0 \\ 4 & 1.0 & 1.4 \\ 5 & 1.4 & 1.8 \\ 6 & 1.8 & 2.2 \\ 7 & 2.2 & 2.6 \\ 8 & 2.6 & 3.0 \\ 9 & 3.0 & 4.0 \\ 10 & 4.0 & 5.0 \\ 11 & 5.0 & 6.0 \\ 12 & 6.0 & 7.5\end{array}$

BETA DECAY ENERGY (MEV/FIS)

5. 40222E-02

5. $05763 E-02$

2. 15015E-O2

3.02747E-02

1. $88445 E-02$

$1.52776 \mathrm{E}-02$

$9.02947 E-03$

$2.82435 E-03$

5.75426E-OA

2. 40575E-O5

2.76628E-06

1. 77 129E - 07

1. $0000 E+14 \mathrm{~N} / \mathrm{CM} * 2-\mathrm{S}$

5. OOOOE+14 N/CM**2-S

$7.2000 E+O T$ SECONDS

1.0000E+06 SECONDS

GAMMA DECAY ENERGY TOTAL DECAY ENERGY (MEV/FIS)

1.50357E-02

1. $48899 E-01$

1. 64012E-02

7.98657E-03

5. 68778E-02

5.67523E-03

$4.91520 E-03$

$6.95132 E-05$

3. 15467E-OS

(AEV/FIS)

6. $90579 E-02$

1.99475E-01

3. 7\$027E-02

$3.82613 E-02$

7.57223E-02

2.09528E-02

1. 39447E-02

2.89386E - 03

$6.06973 E-04$

0 .

2. $40575 E-05$

0.

2. $76628 \mathrm{E}-06$

1. $77129 \mathrm{E}-07$

TOTALS OVER GROUPS

2.02953E-01

2.55891E-O1

4. 58844E-01

FISSION PRODUCT DECAY ENERGY FOR A MIXTURE OF U-235 AND PU-239

PERCENT U-235

PERCENT PU-239

THERMAL FLUX

EPITHERMAL FLUX

OPERATING TIME

$\mathbf{0}$

$\begin{array}{ccc}\text { GRP } & \begin{array}{c}\text { ELO } \\ \text { (MEV) }\end{array} & \begin{array}{c}\text { EHI } \\ \text { (MEV) }\end{array} \\ 1 & 0.0 & .4 \\ 2 & .4 & .8 \\ 3 & .8 & 1.0 \\ 4 & 1.0 & 1.4 \\ 5 & 1.4 & 1.8 \\ 6 & 1.8 & 2.2 \\ 7 & 2.2 & 2.6 \\ 8 & 2.6 & 3.0 \\ 9 & 3.0 & 4.0 \\ 10 & 4.0 & 5.0 \\ 11 & 5.0 & 6.0 \\ 12 & 6.0 & 7.5\end{array}$

TOTALS OVER GROUPS
COOLING TIME

BETA DECAY ENERGY

(MEV/FIS)

1. .55958E-03

1. $40169 E-03$

6. 82085E-04

1.72243E-03

1. $74267 \mathrm{E}-03$

1. 237 15E-03

$7.00417 \mathrm{E}-04$

2. $70177 \mathrm{E}-04$

8.43223E-05

9. 05890E-06

2.27445E-06

1. 26854E-07

9.71 198E-OS
7. $5000 E+01$

2. $5000 E+01$

1. OOOOE+14 N/CM**2-S

$5,0000 \mathrm{E}+14 \mathrm{~N} / \mathrm{Cm} * 2-5$

$7.2000 E+07$ SECONDS

1. OOOOE + OB SECONDS

GANMA DECAY ENERGY (MEV/FIS)

7.00323E-OS

$7.59423 E-03$

2.83293E-04

3. 16643E-04

$1.57313 E-05$

$4.07158 E-05$

1. $88010 E-06$

3.66 127E-07

$9.19320 E-08$

o.

o.

๑.32299E-03
TOTAL DECAY ENERGY (MEV/FIS)

$1.92961 E-03$

- . 99592E-03

$9.65378 E-04$

2. $03907 E-03$

1. $75840 E-03$

1.27787E-O3

$7.02297 E-04$

2.70543E-O4

$8.44143 E-05$

9. OTB9OE-OS

2.27445E-0.

1. 26854E-O7

1. 80350E-02 


\section{APPENDIX B}

\section{ACCURACY ESTIMATE OF ADJUSTED SPECTRAL FITS}

As an aid in estimating the accuracy of the adjusted spectral fits, calculations made with the adjusted fits were separately compared to both aggregate spectral summation results calculated using the CINDER code and ENDF/B-V data and also with the experimental results. Examples of these comparisons are shown graphically in Figs. B-1 through B-5 for the aggregate ganma-ray decay energy from fission products resulting from a pulse irradiation $\left(1 \times 10^{-4} \mathrm{~s}\right)$ of ${ }^{239} \mathrm{Pu}$ with thermal neutrons. (Note that although in figures comparing calculated decay energies with experiment, the experimental points have been reduced to a pulse; those figures showing the deviations take into consideration the actual irradiation times used in the experiments.) The comparisons shown in these figures are for a low-energy group $(0.1-0.2 \mathrm{MeV})$ in Fig. B-1, for two intermediate-energy groups $(0.8-1.0 \mathrm{MeV}$ and $1.4-1.6 \mathrm{MeV})$ in Figs. B-2 and B-3, respectively, and two high-energy groups (2.2-2.4 MeV and 4.0-5.0 MeV) in Figs. B-4 and B-5, respectively. As demonstrated by these figures, the adjusted fits most closely follow the experimental data in the cooling-time range of the experiments, the ENDF/B-V aggregate data for cooling times greater than the range of the experiments, and are extrapolations of the experimental data with and ENDF/B-V "shape" for very early cooling times. Note, however, as can be seen in Fig. B-4, that for high beta- and gama-ray energies, and for long cooling times, the dispersion of the experimental data is so great that the adjusted fits have been forced to fit the ENDF/B-V aggregate data. Also, for the highest energy group $(6.0-7.5 \mathrm{MeV})$, no experimental data were available and the adjusted fits are entirely ENDF/B-V.

As a first step in estimating the reliability of the adjusted fits, we divide the cooling-time range into bins having widths of one decade, except that the last bin is understood to extend to the end of the cooling-tine range $\left(\begin{array}{ll}1 \times 10^{9} & \mathrm{~s}\end{array}\right)$. Averages of deviations of points calculated using the adjusted fits from the aggregate $E N D F / B-V$ pulse points and from the experinental points are next tabulated separately for each energy group and cooling-tine decade for the aggregate betas and gammas from each fissioning nuclide $\left({ }^{235} \mathrm{U}\right.$ and $\left.230 \mathrm{Pu}\right)$ as shown in Tables B-I through B-IV. Combined "accuracy" estinates are then made as follows. 
- For cooling times less than 1 s (i.e., below the experimental range), the combined estimate is taken to be one-half the average deviation for ENDF/B-V.

- For cooling times in the range of $1 \mathrm{~s}-1 \times 10^{4} \mathrm{~s}$, where it is believed that the experimental data are the most accurate, the combined estimate is taken as one-fourth the absolute value of the average deviation for ENDF/B-V plus the average deviation for the experinent.

- For cooling times above $1.0 \mathrm{E}+04 \mathrm{~s}$, but within the range of the experiments, it is assumed that $E N D F / B-V$ data are as valid as the experimental data so the combined estimate is the absolute value of the average deviation for $E N D F / B-V$ plus the absolute value of the experinental deviation. Exceptions to this are the highest energy groups for which the deviations for the experimental data are essentially ignored above $1000 \mathrm{~s}$. (See Fig. B-4.)

- For cooling times above the experimental range, the comp" ned estimate is just the absolute value of the average deviations fron the ENDF/B-V data, as the adjusted fits in this time domain are just fits to the aggregate ENDF/B-V pulse data.

- The minimum combined estimate is taken to be $5 \%$, as this is judged to be the "accuracy" of the ENDF/B-V fits, i.e., no point calculated with the ENDF/B-V fits deviates more than $5 \%$ from an aggregate EHDF/B-V data point.

- The multigroup energy can be rebinned into broader groups For purposes of making uncertainty estimates, as it is generally noted that the experimental gamma-ray decay energy data are lower than the aggregate sumation calculations using ENDF/B-V data for low energies, are in fair agreenent for intermediate energies, and are high for high energies. (The opposite is more or less the case for the betas.) Similarly, wider cooling-time bins can also be assigned.

In accordance with the above, absolute averages of the average deviations were taken over four energy and four cooling-time ranges. The results are displayed in Tables B-V through B-VIII, which also give the bounds of energy and cooling-tine ranges as well as the absolute averages of the deviations.

First, note in compring Tables B-V through B-VIII that the deviations for the gamas are generally higher than those for the betas. This is not surprising since only one experiment (the ORNL) was included in obtaining the adjusted 
beta fits. The deviations in Table B-V and B-VII, therefore, are considered somewhat optimistic.

On the other hand, the large deviations seen in Tables B-VI and B-VIII for the gamas for short cooling times and high energies seem overly pessinistic when examining the numbers in Tables B-II and B-IV. Note in these latter tables that the adjusted fits are extrapolations of closely followed experimental data. As discussed in Ref. 6, we believe that the experimental data are correct for this energy-cooling time domain, and that the data in the ENDF/B-V file are deficient for those nuclides contributing to the decay energy in this donain.

Because of the foregoing, it does not seem unreasonable to make a single estimate of "one-sigma" uncertainties for the adjusted fits for both the betaand gamma-ray decay energies resulting from the thermal pulse irradiation of both ${ }^{235} \mathrm{U}$ and ${ }^{239} \mathrm{Pu}$. This is done by taking simple averages of the deviations in each energy and cooling-time range in Tables $\mathrm{B}-\mathrm{V}$ through $\mathrm{B}-\mathrm{VIII}$. The resulting table appears in Sec. $V$ of the main body of this report. Note that a user may be dissatisfied with the final result of this analysis and may wish to reestimate the uncertainties according to his own needs. If so, Tables B-I through B-IV are available for this task. 
Comparison with ENDF/B-V aggregate data.
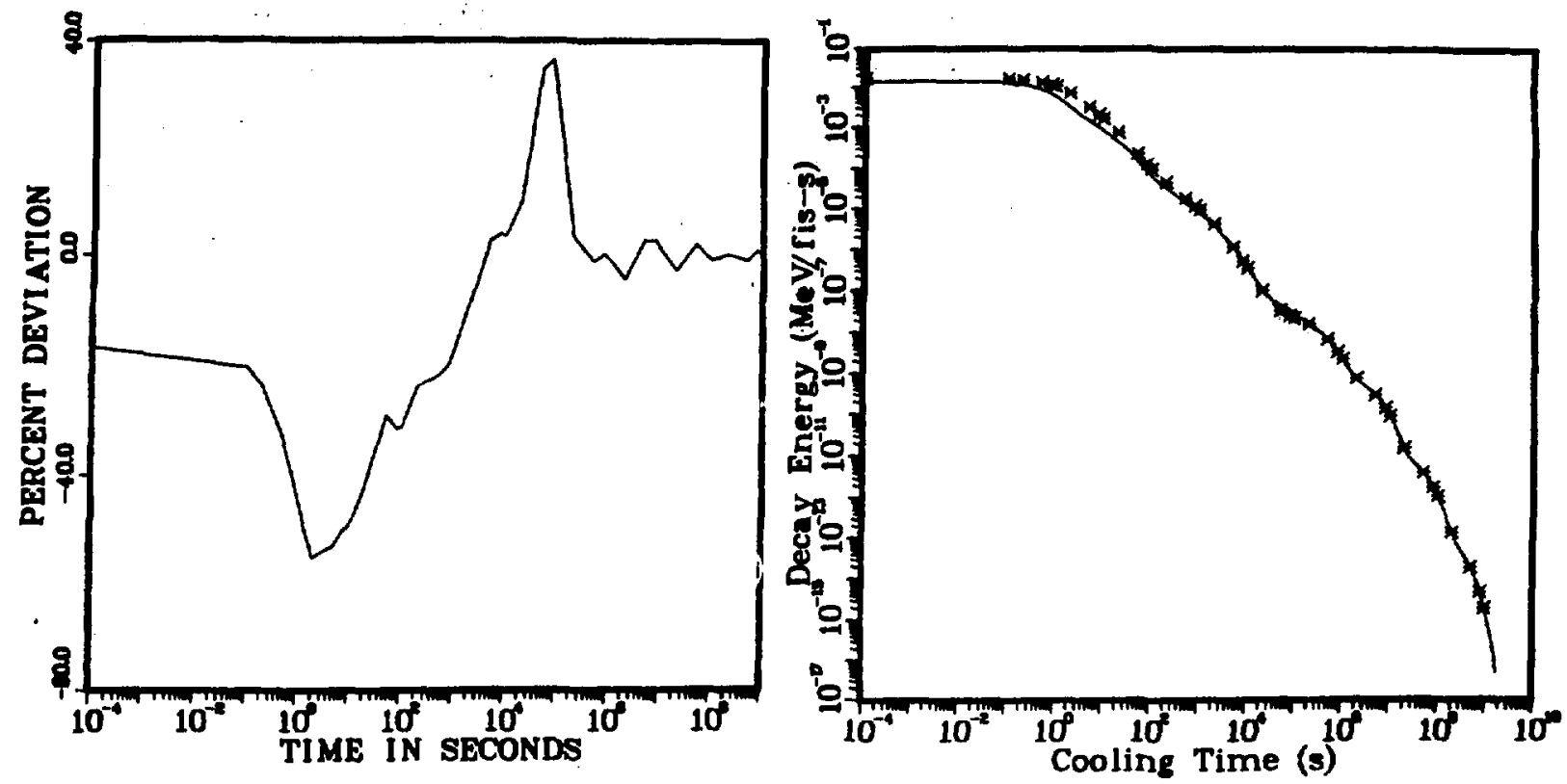

Comparison with experimental data.
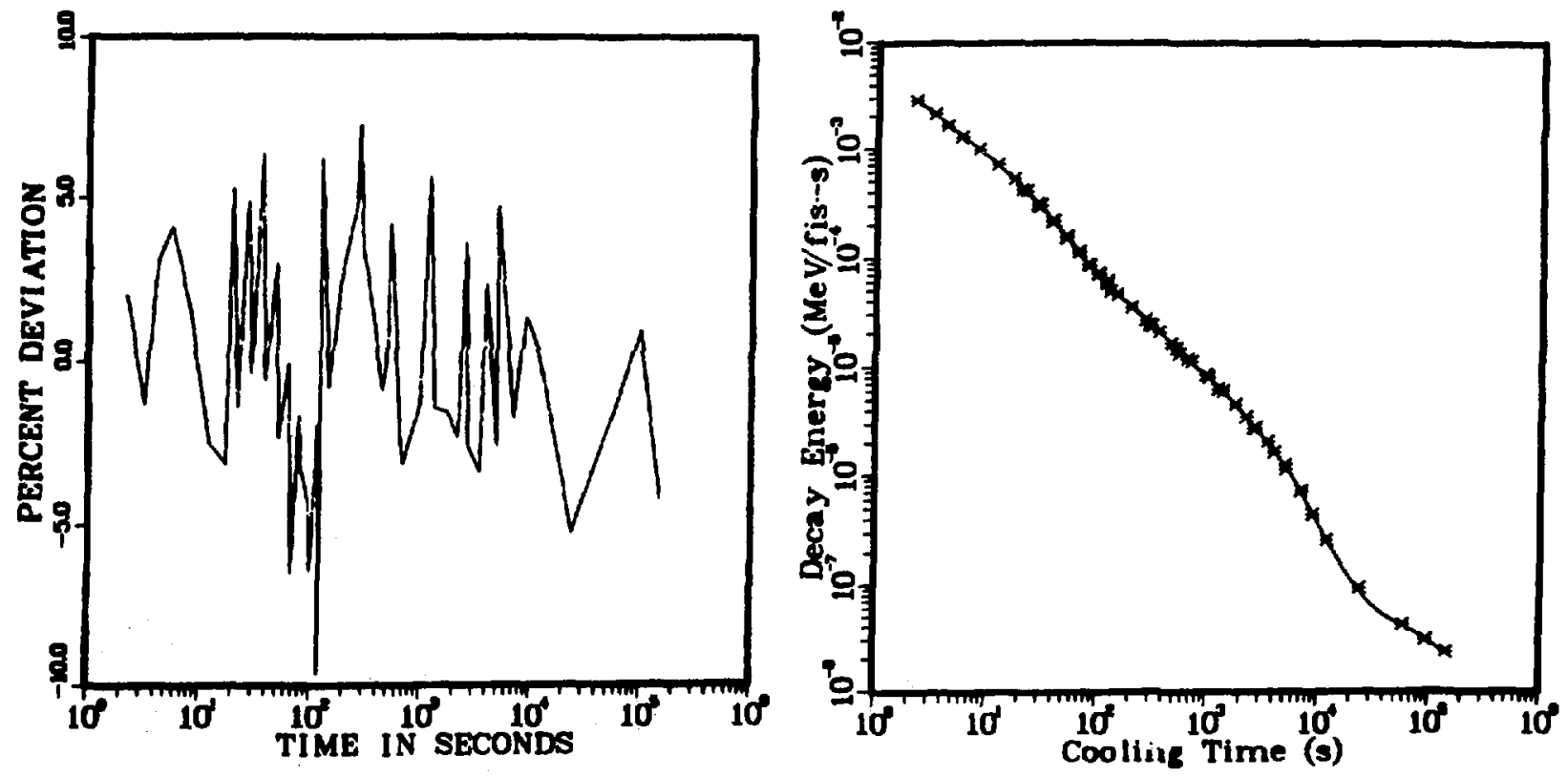

F18. B-1. Comparisons of adjusted fits for Group 2 (0.1-0.2 MeV) 
Comparison with ENDF/B-V aggregate data.
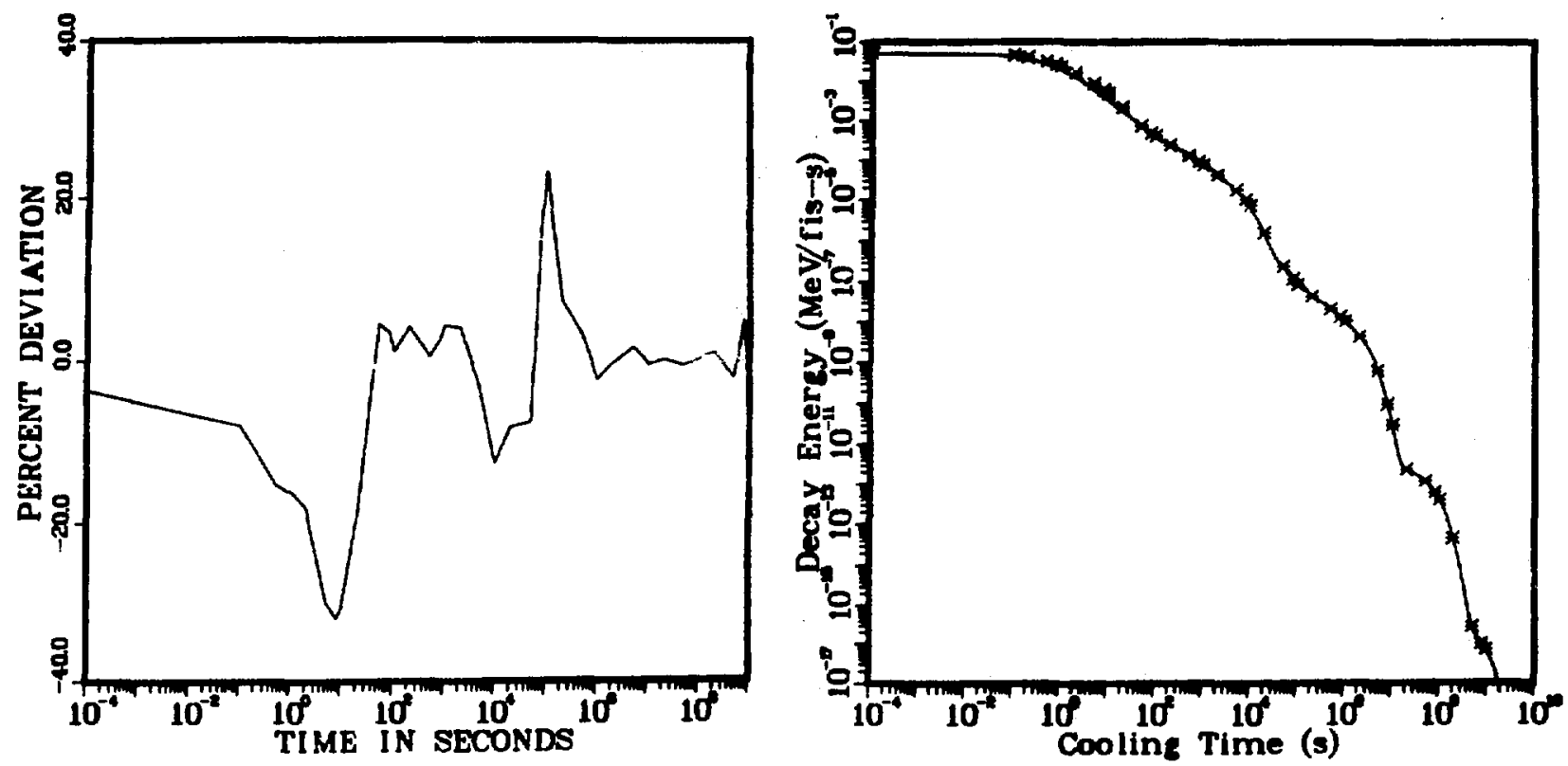

Comparison with experimental data.
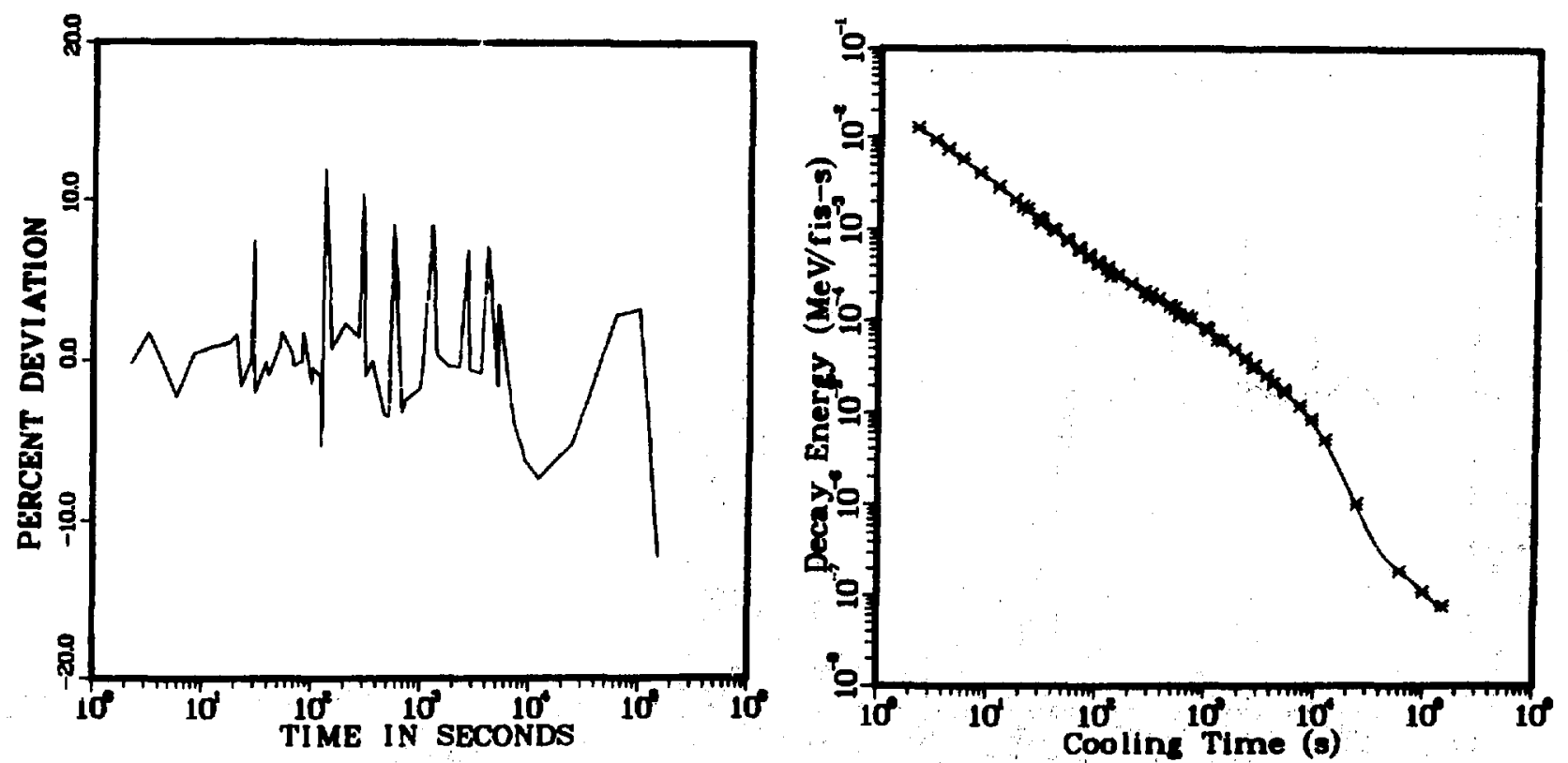

Fig, B-2, Comparisons of adjusted fits for Group $6(0.8-1.0 \mathrm{MeV})$. 
Comparison with ENDF/B-V aggregate data.
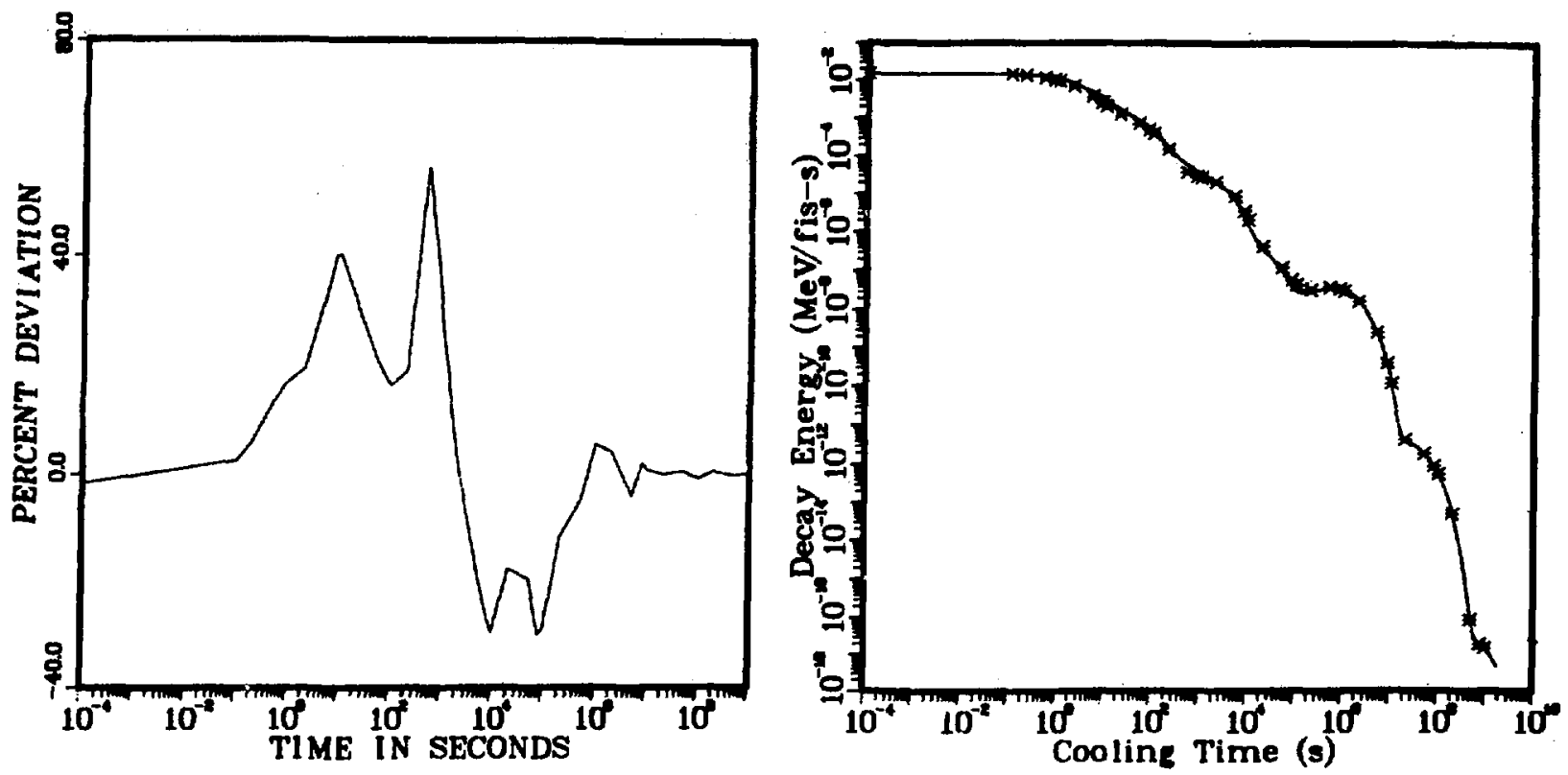

Comparison with experimental data.
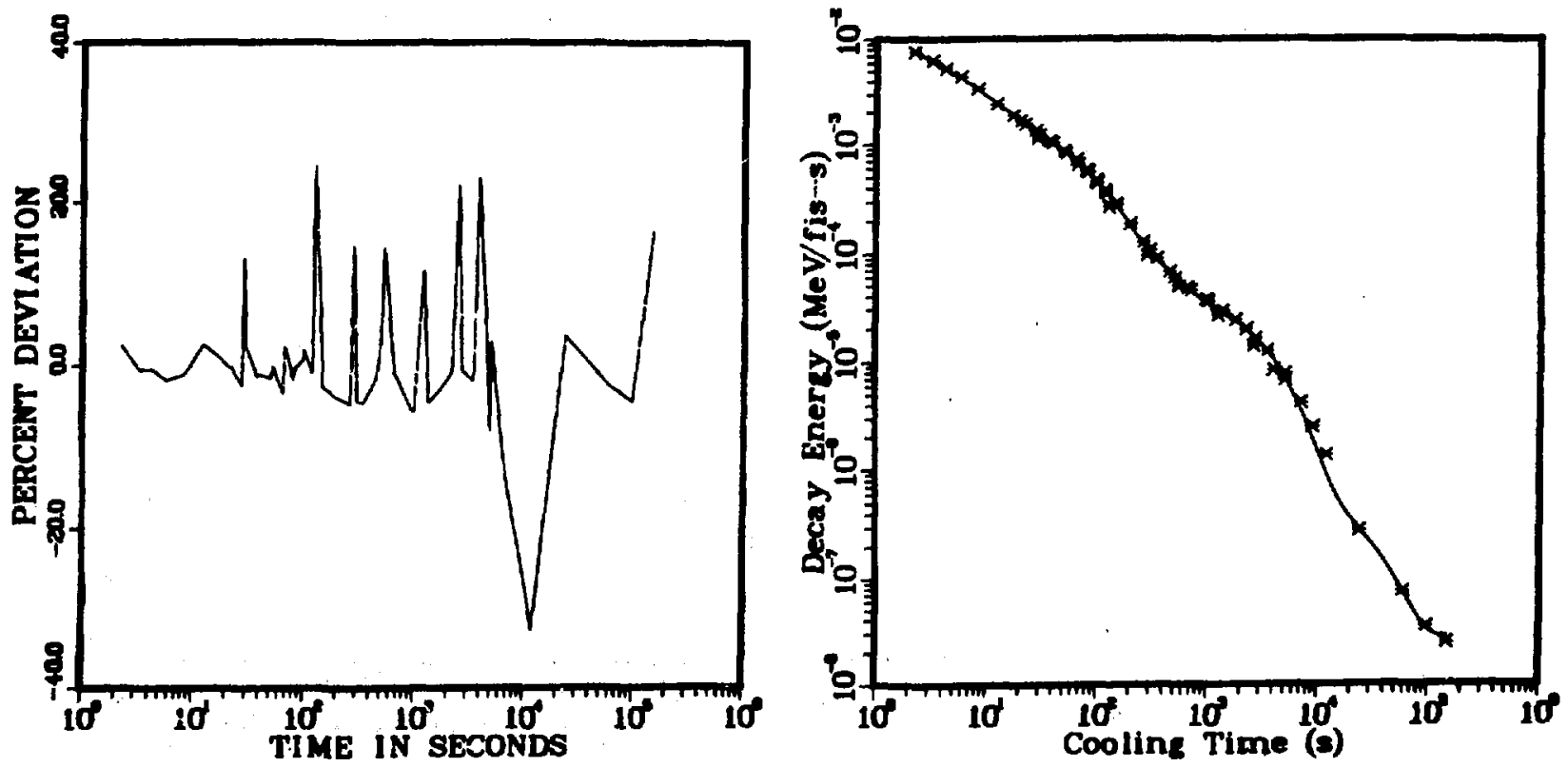

Fig. B-3. Comparisons of adjusted fits for Group $9(1.4-1.6 \mathrm{MeV})$. 
Comparison with ENDF/B-V aggregate data.
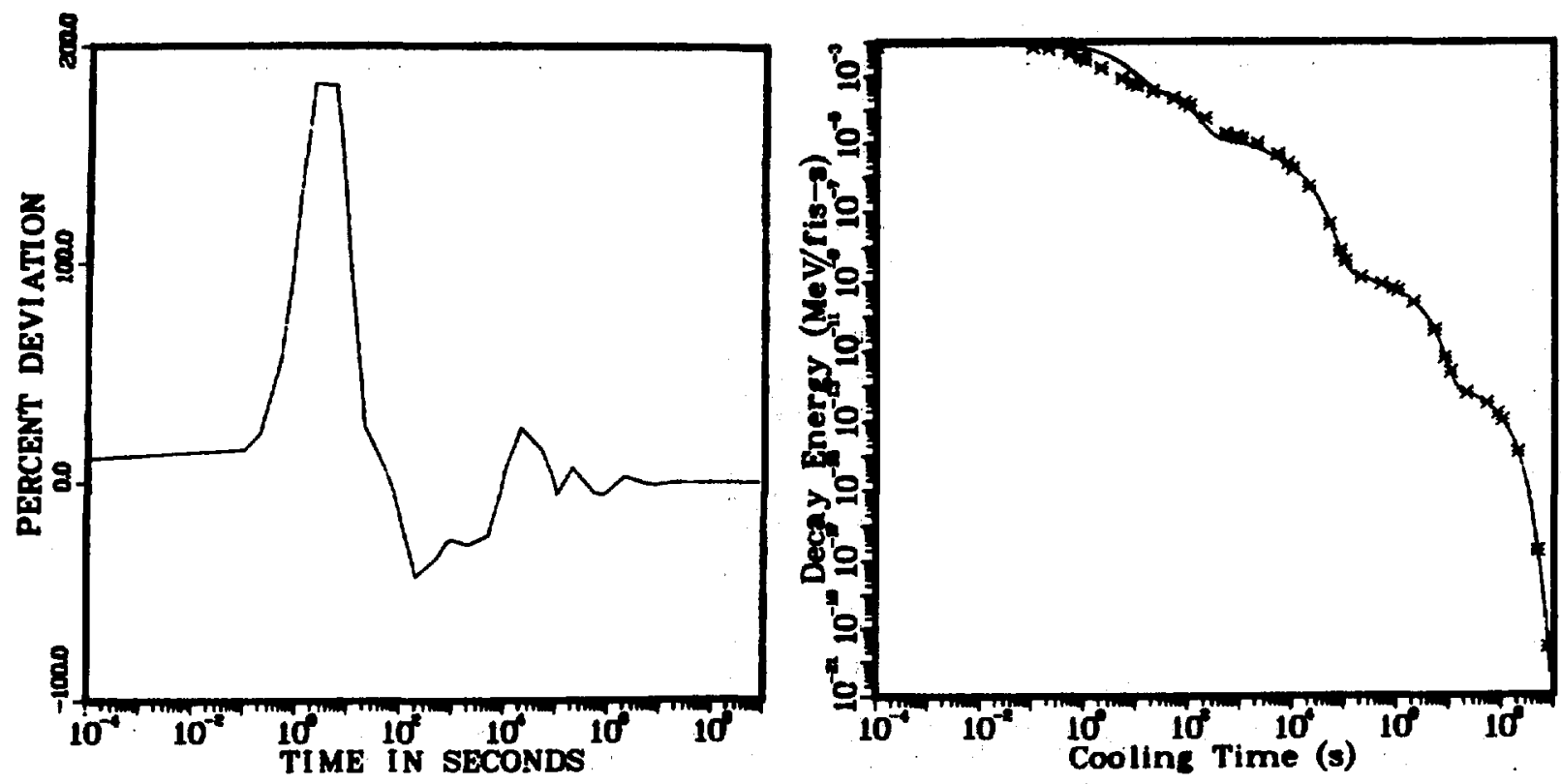

Comparison with experimental data.
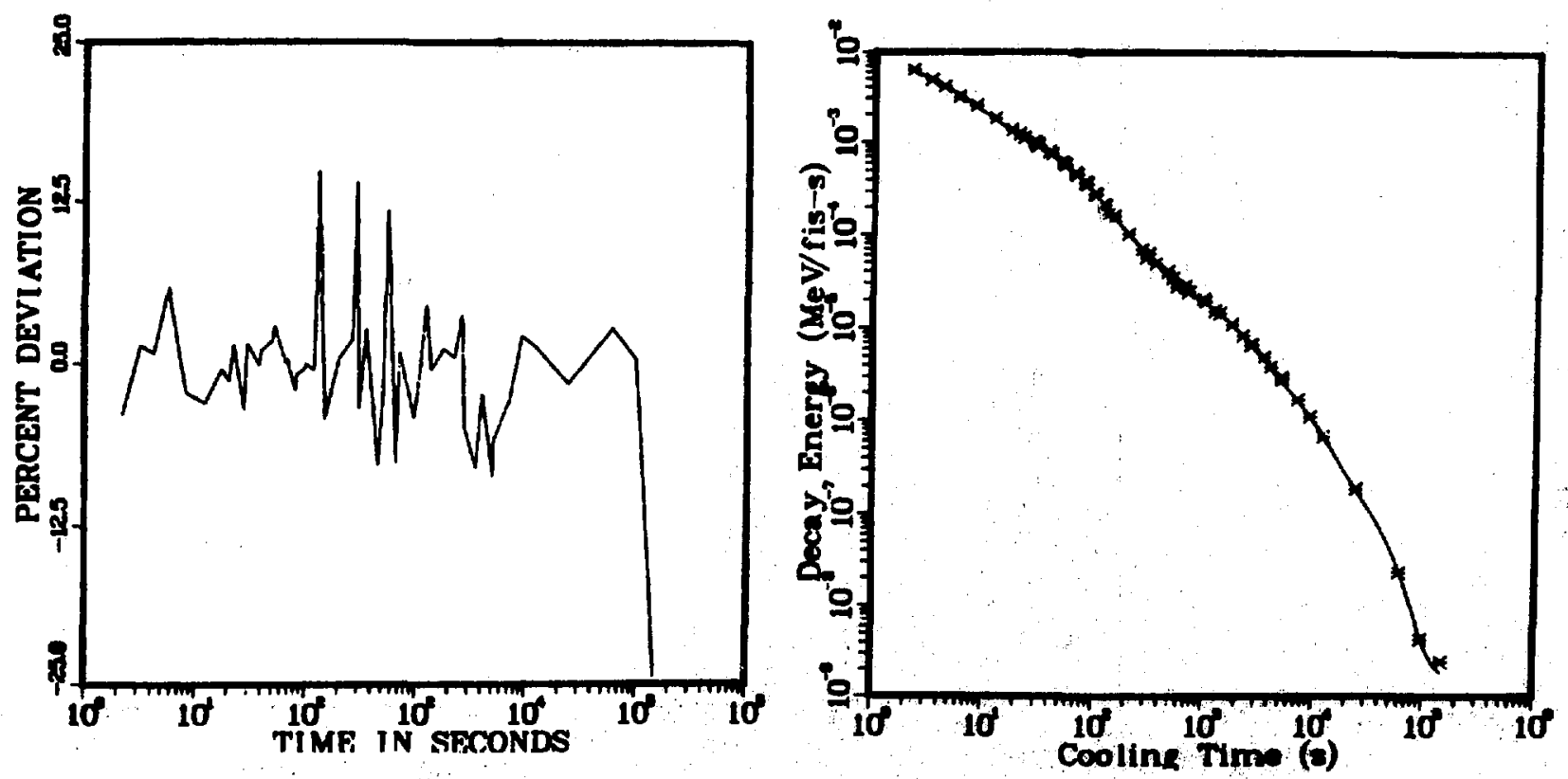

Fig. B-4. Comparisons of adjusted f1ts for Group $13(2,1-1.4 \mathrm{KV})$ 
Comparison with ENDF/B-V aggregate data.
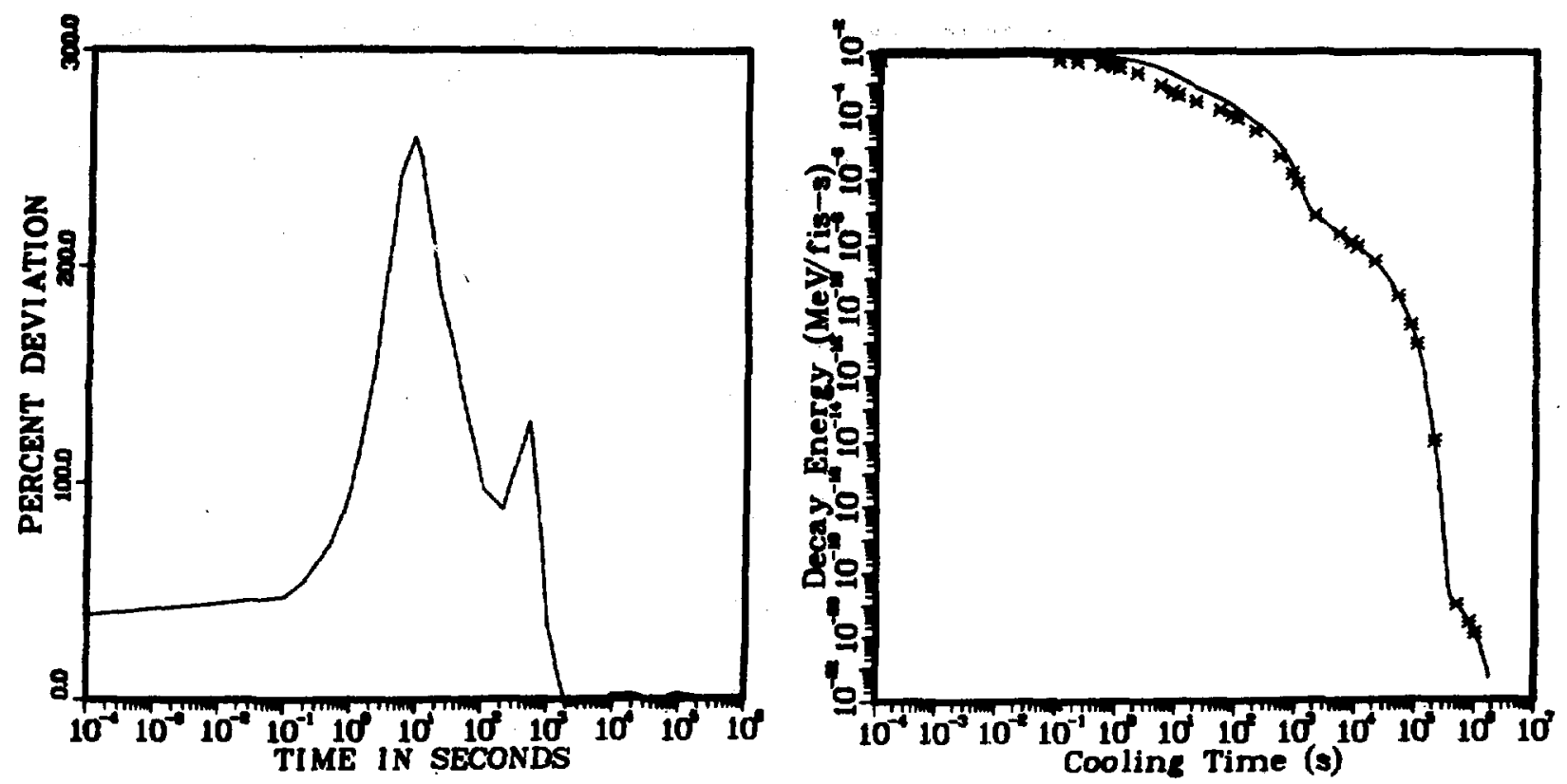

Comparison with experimental data.
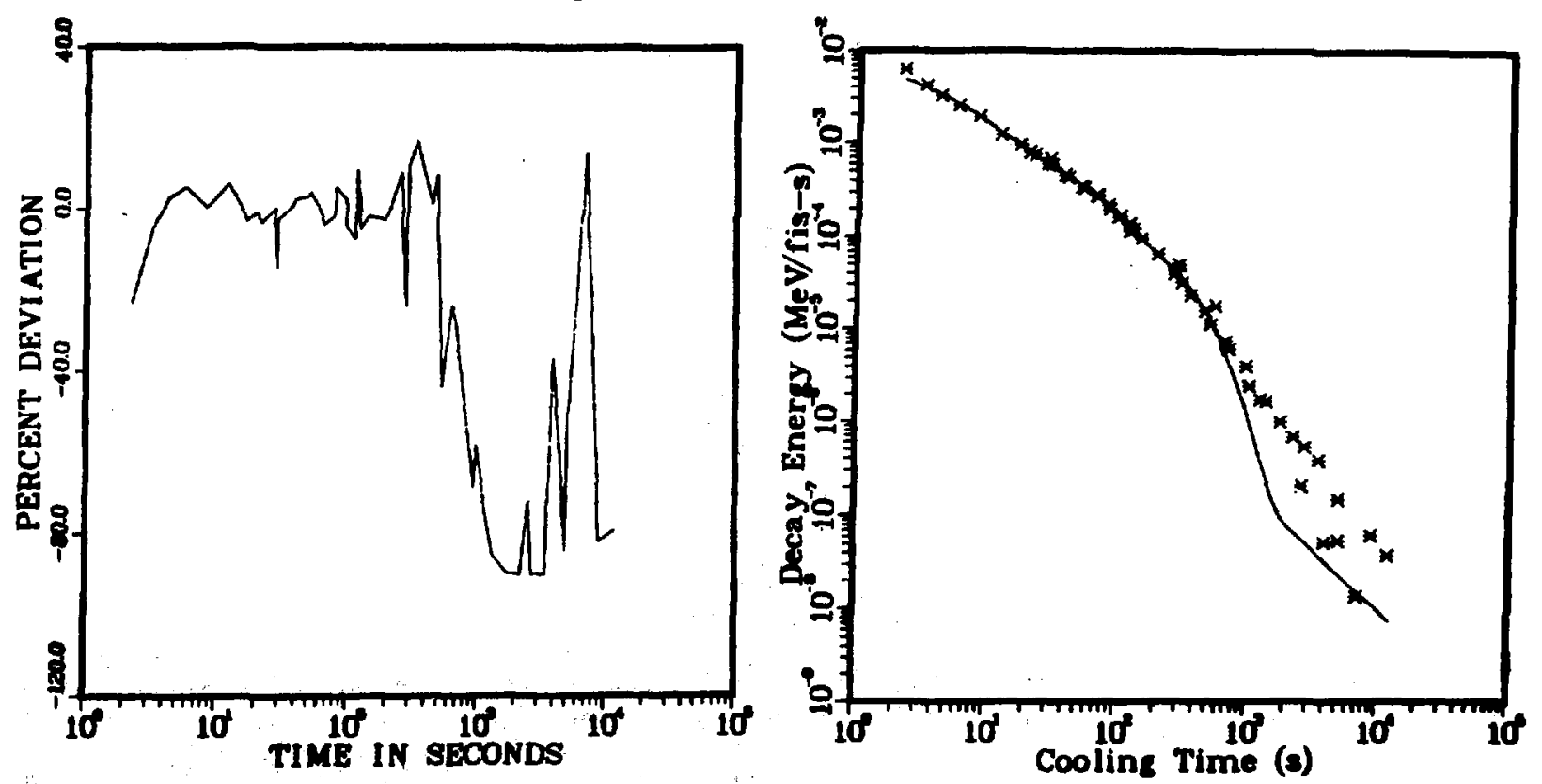

F1g. B-5. Comparisons of adjusted fits for Group $17(4.0-5.0 \mathrm{MeV})$. 
TABLE B-I

aVERAge PERCENT deviations of aDJUSTEd fits fROM ENDF/B-V aNo EXPERIMENTAL DATA FOR U-235 BETAS

\begin{tabular}{|c|c|c|c|c|c|c|c|c|c|c|c|c|c|c|c|c|c|c|}
\hline $\begin{array}{l}\text { COOL TIME } \\
\text { DECADE }\end{array}$ & $\begin{array}{l}\text { GROUP } \\
\text { ENDF }\end{array}$ & $\operatorname{Exp}^{1}$ & $\begin{array}{l}\text { GROUP } \\
\text { ENDF }\end{array}$ & $\operatorname{ExP}^{2}$ & $\begin{array}{l}\text { GROUP } \\
\text { ENDF }\end{array}$ & ExP & $\begin{array}{l}\text { GROUP } \\
\text { ENDF }\end{array}$ & EXP 4 & $\begin{array}{l}\text { GROUP } \\
\text { ENDF }\end{array}$ & $\begin{array}{r}5 \\
\text { EXP }\end{array}$ & $\begin{array}{l}\text { GROUP } \\
\text { ENDF }\end{array}$ & $\begin{array}{r}6 \\
\text { EXP }\end{array}$ & $\begin{array}{l}\text { GROUP } \\
\text { ENDF }\end{array}$ & $\begin{array}{l}P^{7} 7 \\
\text { EXP }\end{array}$ & $\begin{array}{l}\text { GROUP } \\
\text { ENDF }\end{array}$ & ${ }_{\text {EXP }}^{8}$ & $\begin{array}{l}\text { GROUP } \\
\text { ENDF }\end{array}$ & $\operatorname{lP}_{\operatorname{Ex}}^{9}$ \\
\hline $1 E-01-.1 E+\infty$ & 128.9 & 0.0 & 40.5 & 0.0 & 30.1 & 0.0 & 34.3 & 0.0 & .7 & 0.0 & -.9 & 0.0 & 1.7 & 0.0 & -10.6 & 0.0 & -4.9 & 0.0 \\
\hline $.1 E+00-.1 E+01$ & 141.4 & 0.0 & 49.7 & 0.0 & 38.7 & 0.0 & 30.9 & 0.0 & -.0 & 0.0 & 1.1 & 0.0 & 1.7 & 0.0 & -6.5 & 0.0 & 2.2 & 0.0 \\
\hline $.1 E+01-.1 E+02$ & $178.0=$ & -7.6 & $79.4-$ & -9.9 & 56.5 & -.2 & 40.5 & -.3 & $15.1=$ & -1.1 & 12.5 & -.6 & $8.5-$ & -2.1 & -11.9 & .1 & 3.6 & -1.9 \\
\hline $.1 E+02-.1 E+03$ & 109.0 &.$- \mathbf{3}$ & 85.8 & -.4 & $51.0=$ & -4.4 & 48.2 & -.6 & 27.8 & 1.0 & 17.4 & -.9 & $\mathbf{8 . 9}$ & -.9 & -8.5 & .5 & -2.3 & -.8 \\
\hline $.1 E+03-.1 E+04$ & $68.6-$ & -1.9 & 51.5 & 3.2 & 28.4. & -1.2 & 27.3 & .7 & 15.7 & .1 & 11.0 & -.5 & $.9-$ & -2.8 & -.6 & -.1 & -4.5 & .5 \\
\hline 1E+04-. 1E+05 & $66.6=$ & -1.7 & 24.3 & -.1 & 9.2 - & -1.0 & 8.5 & -.0 & 5.8 & .7 & 2.6 & .6 & $-5.1-$ & -1.6 & -5.1 & -.1 & -11.5 & -1.2 \\
\hline $.1 E+05-.1 E+06$ & 18.9 & 0.0 & 8.2 & 0.0 & 2.5 & 0.0 & 1.3 & 0.0 & .0 & 0.0 & -1.0 & 0.0 & -1.8 & 0.0 & -2.8 & 0.0 & -3.6 & 0.0 \\
\hline $.1 E+06-.1 E+07$ & -1.3 & 0.0 & -2.6 & 0.0 & .1 & 0.0 & -.6 & 0.0 & -1.2 & 0.0 & -.2 & 0.0 & -.5 & 0.0 & .0 & 0.0 & .5 & 0.0 \\
\hline $\begin{array}{l}\text { COOL TIME } \\
\text { DECADE }\end{array}$ & $\begin{array}{l}\text { GROUP } \\
\text { ENDF }\end{array}$ & $\begin{array}{l}p 10 \\
\operatorname{EXP}\end{array}$ & $\begin{array}{l}\text { GROUP } \\
\text { ENDF }\end{array}$ & $\begin{array}{l}\text { P } 11 \\
\text { EXP }\end{array}$ & $\begin{array}{l}\text { GROUP } \\
\text { ENDF }\end{array}$ & EXP 12 & $\begin{array}{l}\text { GROUP } \\
\text { ENDF }\end{array}$ & $\begin{array}{l}P \quad 13 \\
\text { EXP }\end{array}$ & $\begin{array}{l}\text { GROUP } \\
\text { ENDF }\end{array}$ & EXP & $\begin{array}{l}\text { GROUP } \\
\text { ENDF }\end{array}$ & $\begin{array}{l}P 15 \\
\text { EXP }\end{array}$ & $\begin{array}{l}\text { GROUP } \\
\text { ENDF }\end{array}$ & $\begin{array}{l}P \quad 16 \\
\text { EXP }\end{array}$ & $\begin{array}{l}\text { GROUP } \\
\text { ENDF }\end{array}$ & $\begin{array}{l}P \quad 17 \\
\text { EXP }\end{array}$ & & \\
\hline $1 E-01-1 E+\infty$ & -.4 & 0.0 & -2.6 & 0.0 & -.5 & 0.0 & -1.5 & 0.0 & -.4 & 0.0 & -.1 & 0.0 & .5 & 0.0 & -46.3 & 0.0 & & \\
\hline $1 E+01-.1 E+02$ & 1.2 & -.1 & 1.0 & -3.0 & -.8 & .5 & -3.3 & -1.2 & $-5.6-$ & -1.6 & -11.3 & -.1 & -18.2 & $2: 7$ & -33.7 & .8 & & \\
\hline $.1 E+02-.1 E+03$ & $-10.5=$ & -1.5 & -13.4 & .1 & $-18.5-$ & $-1: 4$ & -22.5 & -.6 & -26.7 & -.8 & -30.0 & -.3 & -34.6 & -.6 & $-50.1-$ & -5.4 & & \\
\hline $.1 E+03-.1 E+04$ & -12.3 & .9 & -14.3 & -1.7 & -17.6 & $: 3$ & -18.3 & .4 & -25.7 & -.1 & -26.4 & .2 & -35.7 & .4 & $-50.5-$ & -4.5 & & \\
\hline$=1 E+04-1 E+0 S$ & -10.9 & -0 & -11.8 & -2.3 & -10.1 & -.7 & -14.4 & -1.6 & -14.0 & 2.4 & $-10.8=$ & -1.0 & $-16.9=$ & -2.1 & $-20.3-$ & -4.2 & & \\
\hline $.11+0 s-.1 E+06$ & -5.0 & 0.0 & -6.7 & 0.0 & -6.8 & 0.0 & -9.8 & 0.0 & -8.5 & 0.0 & -15.1 & 0.0 & -25.9 & 0.0 & 4.8 & 0.0 & & \\
\hline $.1 E+08-.1 E+07$ & -.0 & 0.0 & -.1 & 0.0 & -.1 & 0.0 & .9 & 0.0 & 2.4 & 0.0 & 3.8 & 0.0 & 20.5 & 0.0 & 1.9 & 0.0 & & \\
\hline
\end{tabular}


TABLE B-II

average percent deviations of aDJusted fits from endF/B-V ANo EXPERimental data for U-235 gaMmas

\begin{tabular}{|c|c|c|c|c|c|c|c|c|c|c|c|c|c|c|c|c|c|}
\hline $\begin{array}{c}\text { COOL TIM } \\
\text { DECADE }\end{array}$ & $\begin{array}{l}\text { ROUP } \\
\text { NDF }\end{array}$ & $P_{\operatorname{Exp}}^{1}$ & $\begin{array}{l}\text { GROUP } \\
\text { ENDF }\end{array}$ & $\exp ^{2}$ & $\begin{array}{l}\text { GROUP } \\
\text { ENDF }\end{array}$ & ${ }^{P} \underset{\text { EXP }}{3}$ & $\begin{array}{l}\text { GROUF } \\
\text { ENDF }\end{array}$ & $\operatorname{Exp}$ & $\begin{array}{l}\text { GROUP } \\
\text { ENDF }\end{array}$ & EXP & $\begin{array}{l}\text { GROUP } \\
\text { ENOFF }\end{array}$ & PXP & & ${ }^{P}{ }^{7}{ }^{7}$ & & $=$ & $\begin{array}{l}\text { GROUP } 9 \\
\text { ENDF EXP }\end{array}$ \\
\hline $1-.1 E+\infty$ & & 0.0 & -39.4 & 0.0 & -67.9 & 0.0 & -7.6 & 0.0 & -40.0 & 0.0 & .7 & 0.0 & 0.6 & 0.0 & 1.6 & 0.0 & -.1 \\
\hline $1 E+\infty 0-.1 E+01$ & 14.1 & 0.0 & -35.9 & 0 & -63.6 & 0.0 & -1 & 0.0 & -42.9 & 0.0 & .1 & 0.0 & B.7 & 0.0 & -8.4 & 0.0 & -3.0 \\
\hline $1 E+01-.1 E+02$ & 31.1 & -.8 & -36.6 & -.9 & -56.8 & .6 & -29.1 & .0 & -40.9 & .4 & -17.9 & -.9 & $47.3=$ & -1.7 & -7.7 & 3.1 & $2.7-2.4$ \\
\hline $1 E+02-.1 E+03$ & -25.2 & -.3 & -11.5 & .0 & -37.4 & -.0 & -9.5 & -.2 & & -.6 & -1.4 & -.1 & $43.4-$ & -1.3 & 9.3 & -.9 & 7.2 \\
\hline $1 E+03-.1 E+04$ & -30.2 & -.5 & -4.9 & 2.1 & -7.1 & 2.5 & -7.5 & 4.1 & -4.9 & 1.1 & $\mathbf{s . 0}$ & .7 & $11.9-$ & -2.1 & 5.5 & 1.6 & 5.4 \\
\hline $1 E+04-.1 E+03$ & .1 & .2 & -.9 & 4.2 & 19.9 & 6.2 & -12.2 & 11.3 & -2.7 & 2.1 & $.7-$ & -1.8 & -13.9 & 6.0 & 4.5 & -1.7 & -10.725 .6 \\
\hline $1 E+0 S-.1 E+06$ & 1.4 & -4.0 & 28.6 & 4.4 & $-6.5-$ & -4.8 & -9.9 & 1.5 & -4.6 & .2 & 2.0 & $-4.9-$ & $-13.9-$ & -1.7 & -10.7 & -9.2 & 7.7 \\
\hline $1 E+06-.1 E+07$ & -8.6 & 0.0 & 0.5 & 0.0 & -8.3 & 0.0 & -2.6 & 0.0 & -1.7 & 0.0 & 3.6 & 0.0 & -4.0 & 0.0 & -7.0 & 0.0 & -9.8 \\
\hline & $\begin{array}{l}\text { ERoup } \\
\text { ENDF }\end{array}$ & & $\begin{array}{l}\text { GROUP } \\
\text { ENDF }\end{array}$ & $P \underset{\text { EXP }}{11}$ & & $\begin{array}{r}12 \\
\text { EXP }\end{array}$ & & & & IP $\begin{array}{r}14 \\
\text { EXP }\end{array}$ & & $\begin{array}{l}15 \\
\text { EXP }\end{array}$ & & $\begin{array}{l}10 \quad 16 \\
=\operatorname{EXP}\end{array}$ & & $\begin{array}{l}J P \quad 17 \\
=\text { EXP }\end{array}$ & $\begin{array}{l}\text { GROUP } 18 \\
\text { ENDF EXP }\end{array}$ \\
\hline $1 E-01-.1 E+\infty$ & & 0.0 & 0.0 & & & 0.0 & & 0.0 & & 0.0 & & 0.0 & & 0.0 & & 0.0 & 0.0 \\
\hline $1 E+\infty 0^{-}$ & 110.5 & 0.0 & 71.0 & 0.0 & 125.3 & 0.0 & 87.3 & 0.0 & 110.9 & 0.0 & 100.7 & 0.0 & 7.1 & 0.0 & $245.2^{\circ}$ & 0.0 & 262.4 \\
\hline $1 E+01-.1 E+02$ & 97.8 & .7 & 75.4 & .7 & 234.4 & $-1.4=$ & 215.8 & $-3.6=$ & 203.3 & -1.6 & 130.8 & .6 & 76.6 & -.0 & 224.7 & -4.2 & $204.9-2.2$ \\
\hline $.1 E+02-.1 E+03$ & 60.7 & -.6 & $96.4-$ & -1.1 & $157.1=$ & -1.5 & 164.8 & -.8 & 108.3 & -.1 & 91.7 & -.4 & & -.4 & 109.2 & -1.7 & 124.6 \\
\hline $.1 E+03-.1 E+04$ & 31.0 & 5.8 & 64.9 & 7.4 & 21.3 & 6.5 & 43.4 & 5.4 & 25.7 & 4.5 & 13.6 & 5.9 & 28.4 & 9.8 & 01.0 & 9.1 & 105.7 \\
\hline $1 E+04-.1 E$ & 6.3 & 11.0 & 19.71 & 19.5 & -15.32 & 23.3 & -20.3 & 27.2 & -6.4 & 5.8 & -2.7 & 8.8 & -4.3 & 3.7 & 8.2- & -24.9 & $9.0-61.3$ \\
\hline (2) & 2.6 & 7.1 & 10.00 & & -29.3 & 0.0 & $-27 \%$ & 34.2 & 44.3 & 3.9 & & 5 & & 0.0 & -.3 & 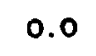 & -.30 .0 \\
\hline $1 E+06-.1 E+07$ & 7.4 & 0.0 & 24.6 & 0.0 & -18.7 & 0.0 & -.2 & 0.0 & 3.2 & 0.0 & 63.2 & 0.0 & 20.4 & 0.0 & -1.0 & 0.0 & $0.0 \quad 0.0$ \\
\hline
\end{tabular}


AVERAGE PERCENT deVIATIONS OF ADUUSTEd fits FROM ENDF/B-V AND EXPERIMENTAL DATA FDR PU-239 BETAS

\begin{tabular}{|c|c|c|c|c|c|c|c|c|c|c|c|c|c|c|c|c|c|c|}
\hline $\begin{array}{l}\text { COOL TIME } \\
\text { DECADE }\end{array}$ & $\begin{array}{l}\text { EROU } \\
\text { ENDF }\end{array}$ & EXP 1 & $\begin{array}{l}\text { EROUP } \\
\text { ENDF }\end{array}$ & ${ }^{P}{ }^{2}{ }^{2}$ & $\begin{array}{l}\text { GROUP } \\
\text { ENDF }\end{array}$ & $P_{\text {EXP }}^{3}$ & $\begin{array}{l}\text { GROUP } \\
\text { ENDF }\end{array}$ & EXP & $\begin{array}{l}\text { GROUP } \\
\text { ENDF }\end{array}$ & ExP & $\begin{array}{l}\text { GROUP } \\
\text { ENDF }\end{array}$ & $P_{\operatorname{EXP}}^{6}$ & $\begin{array}{l}\text { GROUP } \\
\text { ENDF }\end{array}$ & $P_{\operatorname{Exp}}^{7}$ & $\begin{array}{l}\text { GROUP } \\
\text { ENDF }\end{array}$ & $P_{\text {EXP }}^{8}$ & $\begin{array}{l}\text { GROUP } \\
\text { ENDF }\end{array}$ & EXP \\
\hline $.1 E-01-.1 E+\infty$ & 15.4 & 0.0 & 51.6 & 0.0 & 28.5 & 0.0 & -1.7 & 0.0 & .6 & 0.0 & .7 & 0.0 & .1 & 0.0 & -.2 & 0.0 & .4 & 0.0 \\
\hline $.1 E+\infty 0-.1 E+01$ & 31.7 & 0.0 & 58.8 & 0.0 & 32.6 & 0.0 & 9.8 & 0.0 & 4.8 & 0.0 & 1.8 & 0.0 & 1.3 & 0.0 & -.4 & 0.0 & 2.3 & 0.0 \\
\hline $.1 E+01-.1 E+02$ & $100.8-$ & 17.9 & $99.7-1$ & 10.8 & $61.8=$ & -8.1 & 52.1 & .8 & 27.1 & -.8 & $11.7=$ & -5.9 & $11.4=$ & -2.9 & -4.9 & 1.9 & 9.2 & -.3 \\
\hline $.1 E+02-.1 E+03$ & 143.3 & .5 & 134.9 & $\mathbf{8 . 0}$ & 90.7 & 5.8 & 70.2 & -.0 & 41.0 & 1.7 & 23.3 & 1.7 & 14.4 & 1.6 & $-2.6=$ & -1.0 & .8 & .6 \\
\hline $.1 E+03-.1 E+04$ & 115.0 & -.4 & 20.7 & 1.1 & 55.4 & 1.6 & 37.0 & -.2 & 25.3 & 3.0 & 10.7 & .0 & 1.6 & -.3 & -5.7 & -.3 & -11.1 & -.5 \\
\hline $.1 E+04-.16+05$ & 92.2 & -.4 & $42.4=$ & -2.1 & 26.2 & -1.2 & 11.1 & -.0 & 3.0 & .5 & -5.5 & .3 & -8.5 & 2.1 & -11.8 & 2.0 & -14.0 & 2.0 \\
\hline $.1 E+05-.1 E+06$ & 15.9 & 0.0 & 15.6 & 0.0 & 7.4 & 0.0 & 1.7 & 0.0 & -2.1 & 0.0 & -3.8 & 0.0 & -5.2 & 0.0 & -1.7 & 0.0 & -2.3 & 0.0 \\
\hline - 1E+08- . 1E+07 & .8 & 0.0 & -2.7 & 0.0 & -2.4 & 0.0 & -.4 & 0.0 & .2 & 0.0 & .8 & 0.0 & .0 & 0.0 & .2 & 0.0 & -.9 & 0.0 \\
\hline $\begin{array}{l}\text { coot trme } \\
\text { Oecaot }\end{array}$ & $\begin{array}{l}\text { enou } \\
\text { ENof }\end{array}$ & $\begin{array}{r}10 \\
\text { EXP }\end{array}$ & $\begin{array}{l}\text { GROUP } \\
\text { ENOF }\end{array}$ & EXP & $\begin{array}{l}\text { GROUP } \\
\text { ENDF }\end{array}$ & EXP 12 & $\begin{array}{l}\text { EROUF } \\
\text { ENOF }\end{array}$ & EXP 13 & $\begin{array}{l}\text { GROUP } \\
\text { ENDF }\end{array}$ & EXP & $\begin{array}{l}\text { GROUF } \\
\text { ENUF }\end{array}$ & PXP & $\begin{array}{l}\text { GROUP } \\
\text { ENDF }\end{array}$ & EXP 16 & $\begin{array}{l}\text { GROUF } \\
\text { ENDF }\end{array}$ & $\begin{array}{r}17 \\
\text { EXP }\end{array}$ & & \\
\hline $.1 E-01-.1 E+\infty$ & 5.4 & 0.0 & 3.8 & 0.0 & 2.5 & 0.0 & 3.0 & 0.0 & 2.2 & 0.0 & $\because 5$ & 0.0 & .4 & 0.0 & 3.8 & 0.0 & & \\
\hline $.1 E+\infty 0-.1 E+01$ & .0 & c.o & 4.0 & 0.0 & 1.2 & 0.0 & 1.9 & 0.0 & -.0 & 0.0 & .4 & 0.0 & -3.9 & 0.0 & -15.1 & 0.0 & & \\
\hline $.1 E+01-.1 E+02$ & 6.7 & -.5 & 7.5 & -.3 & 7.4 & -.4 & 1.7 & -2.3 & -.6 & -.3 & -6.8 & .2 & -21.6 & -.4 & -36.5 & -2.0 & & \\
\hline $.1 E+02-.1 E+03$ & -5.0 & .6 & -10.2 & .5 & -15.3 & 1.0 & -20.1 & 1.4 & -27. & .4 & -35.2 & .1 & -48.3 & .7 & -63.0 & -.3 & & \\
\hline $.1 E+08-.1 E+04$ & -17.2 & -1.1 & -19.9 & -.2 & -23.1 & -.5 & $-27,8$ & -2.6 & -31.4 & -.9 & -36.5 & -.3 & -60.6 & -2.2 & -65.5 & -7.5 & & \\
\hline $.1[+04-.11+00$ & -17.8 & .7 & -17.3 & 1.1 & -18.0 & .8 & -19.1 & 1.0 & -21.9 & .8 & -19.4 & -.1 & -21.0 & -3.5 & -13.6 & 9.8 & & \\
\hline $.15+\infty-.1$ & -7.0 & 0.0 & -7.2 & 0.0 & -5.7 & 0.0 & -8.4 & 0.0 & -7.7 & 0.0 & -13.4 & 0.0 & 1.0 & 0.0 & 1.1 & 0.0 & & \\
\hline $.1 E+08-$. & -.7 & 0.0 & -1.2 & 0.0 & 3.3 & 0.0 & 1.9 & 0.0 & 5.1 & 0.0 & -.1 & 0.0 & 2.4 & 0.0 & -1.0 & 0.0 & & \\
\hline
\end{tabular}




\section{TABLE B-IV}

AVERAGE PERCENT DEVIATIONS OF ADUUSTED FITS FROM ENOF/B-V AND EXPERIMENTAL DATA FOR PU-239 GAMMAS

\begin{tabular}{|c|c|c|c|c|c|c|c|c|c|c|c|c|c|c|c|c|}
\hline $\begin{array}{c}\text { COOL TIME } \\
\text { DECADE }\end{array}$ & $\begin{array}{l}\text { CROUP } \\
\text { ENDFF }\end{array}$ & ${ }^{P}=\begin{array}{l}1 \\
\text { EXP }\end{array}$ & $\begin{array}{l}\text { GROUP } \\
\text { ENDF }\end{array}$ & $P={ }^{2}{ }^{2}$ & $\begin{array}{l}\text { GROUP } \\
\text { ENDF }\end{array}$ & EXP & $\begin{array}{l}\text { GROUP } \\
\text { ENDF }\end{array}$ & EXP & $\begin{array}{l}\text { GROUF } \\
\text { ENDF }\end{array}$ & EXP & $\begin{array}{l}\text { GROUP } \\
\text { ENDF }\end{array}$ & $P_{\text {EXP }}^{6}$ & $\begin{array}{l}\text { GROUP } \\
\text { ENDFF }\end{array}$ & ${ }^{2}{ }_{\operatorname{EXP}}^{7}$ & $\begin{array}{l}\text { GFOUP } 8 \\
\text { ENDF EXP }\end{array}$ & $\begin{array}{l}\text { GROUP } 9 \\
\text { ENDF EXP }\end{array}$ \\
\hline $1 E-01-.1 E+\infty$ & 05.0 & 0.0 & $-\$ 6.8$ & 0.0 & -68.4 & 0.0 & -11.2 & 0.0 & -36.6 & 0.0 & -3.6 & 0.0 & 7.5 & 0.0 & 0.0 & 1.7 \\
\hline$E+\infty 0-.1 E+01$ & 81.0 & .0 & -29.6 & 0.0 & -65.0 & 0.0 & -13.7 & 0.0 & -29.1 & 0.0 & -12.5 & 0.0 & 15.4 & 0.0 & 0.0 & 9.3 \\
\hline $1 E+01-.1 E+02$ & $48.6-$ & -2.8 & -50.8 & 1.9 & -55.1 & 1.1 & -26.4 & -.3 & -32.8 & .9 & -24.0 & -.1 & 46.0 & -.3 & 1.3 & 27.1 \\
\hline $1 E+02-.1 E+03$ & -32.2 & 2.5 & -38.1 & -.6 & $-47.7-$ & -2.4 & $-18.6-$ & -1.6 & -41.8 & -.5 & -10.5 & .5 & 58.5 & .3 & 12.8 & 27.5 \\
\hline $1 E+03-.1 E+04$ & -26.7 & -.4 & -24.4 & .6 & -16.6 & -.5 & -14.5 & -.9 & -11.4 & 1.0 & 2.0 & .8 & 22.7 & .4 & 17.9 & 32.5 \\
\hline $1 E+04-.1 E+05$ & .0 & .1 & -5.0 & .2 & 8.8 & .5 & -13.4 & .8 & -4.9 & -.2 & -1.2 & 1.0 & -11.5 & -.7 & -.7 & -4.0 \\
\hline $1 E+05-.1 E+\infty$ & -12.7 & 0.0 & $21.1-$ & -1.4 & 5.4 & .2 & -11.7 & 3.1 & -15.3 & -2.3 & $-2.9-$ & -1.7 & -21.9 & -4.0 & $-19.1-2.1$ & $-24.0-9.0$ \\
\hline $1 E+06-.1 E+07$ & -.1 & 0.0 & 7.9 & 0.0 & -1.7 & 0.0 & -4.2 & 0.0 & -6.9 & 0.0 & 8.3 & 0.0 & -6.7 & 0.0 & -12.3 & -10.8 \\
\hline & $\begin{array}{l}\text { EROUP } \\
\text { ENDF }\end{array}$ & $\begin{array}{r}10 \\
\text { EXP }\end{array}$ & $\begin{array}{l}\text { GNoup } \\
\text { ENDF }\end{array}$ & $\begin{array}{l}11 \\
\text { EXP }\end{array}$ & $\begin{array}{l}\text { CROUP } \\
\text { ENDF }\end{array}$ & $=\begin{array}{l}12 \\
\text { EXP }\end{array}$ & $\begin{array}{l}\text { GROUP } \\
\text { ENDF }\end{array}$ & $\begin{array}{r}13 \\
\text { EXP }\end{array}$ & $\begin{array}{l}\text { GROAN } \\
\text { ENDF }\end{array}$ & P 14 & $\begin{array}{l}\text { GROUP } \\
\text { ENDFF }\end{array}$ & $\begin{array}{l}15 \\
\text { EXP }\end{array}$ & & $\begin{array}{l}16 \\
\text { EXP }\end{array}$ & $\begin{array}{l}\text { GROUP } 17 \\
\text { ENDF EXP }\end{array}$ & $\begin{array}{l}\text { EROUP } 18 \\
\text { ENDF EXP }\end{array}$ \\
\hline $1 E-01-.1 E+\infty 0$ & & 00 & & 0.0 & & 0.0 & 35.0 & 0.0 & & 0.0 & & 0.0 & & 0.0 & 0.02 & 261.2 \\
\hline $1 E+\infty-.1 E+01$ & 78.4 & 0.0 & 17.5 & 0.0 & 163.3 & 0.0 & 82.7 & 0.0 & 239.2 & 0.0 & 107.5 & 0.0 & & 0.0 & 0.02 & 234.4 \\
\hline $1 E+01-.1 E+02$ & 94.5 & -.4 & 85.1 & -.0 & 349.9 & -.7 & 273.7 & .3 & 329.5 & 3.9 & 180.1 & .0 & 76.7 & -.8 & $186.5-3.5$ & $181.1-4.4$ \\
\hline $1 E+02-.1 E+03$ & 76.3 & .1 & 155.2 & $\therefore 0$ & 242.8 & .1 & 214.4 & -.1 & 174.1 & -1.4 & 167.0 & -.1 & 152.5 & -.1 & 171.5 & $154.1^{\prime}-2.3$ \\
\hline $.1 E+03-.1 E+04$ & 47.5 & .7 & 103.9 & .1 & 61.3 & $.0^{\circ}$ & 79.6 & .9 & 57.6 & -.0 & 30.4 & 1.2 & 73.2 & .8 & 96.7 & $78.7-23.2$ \\
\hline $1 E+04-.1 E+05$ & 4.6 & -2.0 & & -.2 & -1.1 & -1.2 & $-12.6-$ & -1.8 & 18.9 & -.3 & $7.9-$ & -6.7 & 21.4 & -2.0 & $7.2-20.0$ & $4.2-86.0$ \\
\hline $1 E+05-.1 E+06$ & -21.0 & 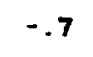 & -6.8 & -.0 & -40.3 & -5.1 & -24.5 & .7 & 5.3 & -2.7 & $36.5-2$ & 21.4 & -34.5 & 0.0 & $\begin{array}{ll}1.2 & 0.0\end{array}$ & -.3 \\
\hline $1 E+08-.1 E+07$ & -3.0 & .0 & 4.3 & 0.0 & -23.9 & 0.0 & 3.4 & 0.0 & 1.0 & 0.0 & 89.3 & 0.0 & -10.4 & 0.0 & .80 .0 & $0.0 \quad 0.0$ \\
\hline
\end{tabular}


TABLE B-V

PERGENT ESTIMATE OF ACCURACY OF ADJUSTED FITS FOR U-235 BETAS

\begin{tabular}{|c|c|c|c|c|}
\hline $\begin{array}{l}\text { ENERGY } \\
\text { RANGES (MEV) }\end{array}$ & $1.0 \varepsilon-02-1.0 \varepsilon+\infty$ & $\begin{array}{l}\text { COOLING TIME } \\
1.0 E+O D-1.0 E+O A\end{array}$ & $\begin{array}{l}\text { RANGES (S) } \\
1.0 E+04-1.0 E+O S\end{array}$ & $1.0 E+06-1.0 E+09$ \\
\hline $0.0-.6$ & 35.8 & 19.5 & 3.0 & 5.0 \\
\hline $.6-1.6$ & 5.0 & 5.0 & 5.0 & 5.0 \\
\hline $1.6-3.0$ & $\mathbf{5 . 0}$ & 5.0 & 5.0 & 5.0 \\
\hline $3.0-7.5$ & 7.6 & 8.9 & 12.0 & 5.0 \\
\hline
\end{tabular}

TABLE B-VI

PERCENT ESTIMATE of accuracy OF ADJUSTED FITS FOR U-235 gammas

ENERGY COOLING TIME RANGES (S)

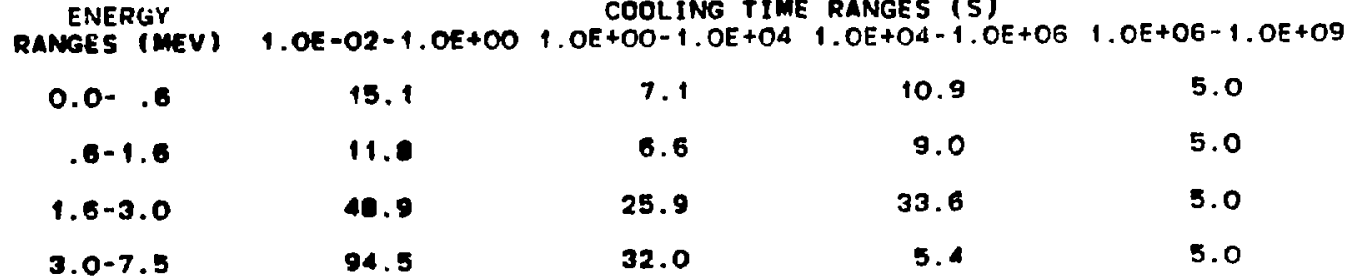

TABLE B-VII

PERCENT ESTIMATE OF ACCURACY OF AOJUSTEO FITS FOR PU-239 BETAS

ENEROY CODLING TIME RANGES (S)

RAMGES (MEV) 1.0E-02-1.0E+00 1.0E+DO-1.0E+04 1.0E+04-1.0E+06 $1.0 E+06-1.0 E+09$
$0.0-.6$
18.2
26. 7
7.4
5.0
.6-1.6
5.0
6.1
5.0
3.0
1.6-3.0
5.0
5. 0
5.0
5.0
3.0-7.5
5.0
11.2
5.0
5.0

TABLE B-VIII

PERCENT ESTIMATE OF ACCURACY OF ADUUSTED FITS FOR PU-239 GAMNAS

ENEREY RANGES (MEV

0.0- . 6

$.6-1.6$

1.6-3.0

3.0-7.5
COOL ING TIME RANOES ( 5 )

$1.0 E-02-1.0 E+001.0 E+00-1.0 E+041.0 E+04-1.0 E+061.0 E+06-1.0 E+09$

21.9

7.8

8. 7

5. 0

5.8

49.7

5.6

14.7

5.0

30.0

37.2

55.0
24.2

5.0

7.9

5.0 